CERN-PH-TH/2004-020

TUM-HEP-540/04

MPP-2004-14

hep-ph/0402112

\title{
Anatomy of Prominent $B$ and $K$ Decays and Signatures of CP-Violating New Physics in the Electroweak Penguin Sector
}

\author{
Andrzej J. Buras, ${ }^{a}$ Robert Fleischer, ${ }^{b}$ Stefan Recksiegel ${ }^{a}$ and Felix Schwab ${ }^{c, a}$ \\ ${ }^{a}$ Physik Department, Technische Universität München, D-85748 Garching, Germany \\ ${ }^{b}$ Theory Division, Department of Physics, CERN, CH-1211 Geneva 23, Switzerland \\ ${ }^{c}$ Max-Planck-Institut für Physik - Werner-Heisenberg-Institut, D-80805 Munich, \\ Germany
}

\begin{abstract}
The recent observation of $B_{d} \rightarrow \pi^{0} \pi^{0}$ at the $B$ factories with a surprisingly large branching ratio represents a challenge for theory, and complements the amazingly small $B_{d} \rightarrow \pi^{+} \pi^{-}$rate. We point out that all puzzling $B \rightarrow \pi \pi$ features can be accommodated in the Standard Model (SM) through non-factorizable hadronic interference effects, extract the relevant parameters, and predict the CP asymmetries of $B_{d} \rightarrow \pi^{0} \pi^{0}$. Using then $S U(3)$ flavour-symmetry and plausible dynamical assumptions, we fix the hadronic $B \rightarrow \pi K$ parameters through their $B \rightarrow \pi \pi$ counterparts, and determine the CKM angle $\gamma$, with a result in remarkable accordance with the usual fits for the unitarity triangle. We may then analyse the $B \rightarrow \pi K$ system in the SM, where we find agreement with the experimental picture, with the exception of those observables that are significantly affected by electroweak (EW) penguins, thereby suggesting new physics (NP) in this sector. Indeed, a moderate enhancement of these topologies and a large $\mathrm{CP}$-violating NP phase allow us to describe any currently observed feature of the $B \rightarrow \pi K$ modes, and to predict the CP-violating $B_{d} \rightarrow \pi^{0} K_{\mathrm{S}}$ observables. If we then restrict ourselves to a specific scenario where NP enters only through $Z^{0}$ penguins, we obtain a link to rare $K$ and $B$ decays, where the most spectacular NP effects are an enhancement of the $K_{\mathrm{L}} \rightarrow \pi^{0} \nu \bar{\nu}$ rate by one order of magnitude with $\operatorname{BR}\left(K_{\mathrm{L}} \rightarrow \pi^{0} \nu \bar{\nu}\right) \approx 4 \mathrm{BR}\left(K^{+} \rightarrow \pi^{+} \nu \bar{\nu}\right)$, $\operatorname{BR}\left(K_{\mathrm{L}} \rightarrow \pi^{0} e^{+} e^{-}\right)=\mathcal{O}\left(10^{-10}\right),(\sin 2 \beta)_{\pi \nu \bar{\nu}}<0$, and a large forward-backward $\mathrm{CP}$ asymmetry in $B_{d} \rightarrow K^{*} \mu^{+} \mu^{-}$. We address also $\varepsilon^{\prime} / \varepsilon$ and other prominent decays, including $B \rightarrow \phi K$ and $B \rightarrow J / \psi K$ modes.
\end{abstract}

February 2004 



\section{Contents}

1 Introduction $\quad 1$

2 A Simple Scenario for New Physics 4

2.1 General Structure . . . . . . . . . . . . . . . . . . . . . . 4

2.2 Going Beyond MFV . . . . . . . . . . . . . . . . . . . . 7

2.3 Relation Between Rare Decays and $B \rightarrow \pi K$ Modes . . . . . . . . . . . 8

2.4 CKM Parameters . . . . . . . . . . . . . . . . . . . . . 99 9

2.5 Summary . . . . . . . . . . . . . . . . . . . . 10

3 The $B \rightarrow \pi \pi$ System $r$

3.1 Basic Formulae . . . . . . . . . . . . . . . . . . . . . . . . 10

3.2 Determination of the Hadronic Parameters . . . . . . . . . . . . . . . 13

3.3 Interpretation of the Hadronic Parameters . . . . . . . . . . . . . . . . 15

3.3.1 Insights into the Substructure of $x e^{i \Delta} \ldots \ldots \ldots$. . . . . . . 16

3.3.2 Probing Penguin Annihilation and Exchange Topologies through the $B_{d} \rightarrow K^{+} K^{-}, B_{s} \rightarrow \pi^{+} \pi^{-}$System . . . . . . . . . . 18

3.4 Determination of $\gamma \ldots \ldots \ldots \ldots$. . . . . . . . . . . . . . . . . . 19

3.5 Prediction of CP Violation in $B_{d} \rightarrow \pi^{0} \pi^{0} \ldots \ldots$. . . . . . . . . . . . . 22

3.6 EW Penguin Contributions . . . . . . . . . . . . . . . . . . . . 23

3.7 Summary .............................. 24

4 The $B \rightarrow \pi K$ System $\quad 25$

4.1 Basic Formulae . . . . . . . . . . . . . . . . . . . . . . . 25

4.2 Determination of the Hadronic Parameters . . . . . . . . . . . . . . . 26

$4.2 .1 \rho_{\mathrm{c}} e^{i \theta_{\mathrm{c}}} \ldots \ldots \ldots \ldots \ldots \ldots \ldots$

4.2.2 $r_{\mathrm{c}} e^{i \delta_{\mathrm{c}}}, r e^{i \delta}$ and $\rho_{\mathrm{n}} e^{i \theta_{\mathrm{n}}} \ldots \ldots \ldots \ldots \ldots . \ldots . \ldots . \ldots 27$

4.2.3 Further Theoretical Aspects of $\rho_{\mathrm{c}} e^{i \theta_{\mathrm{c}}}$. . . . . . . . . . . . . . 29

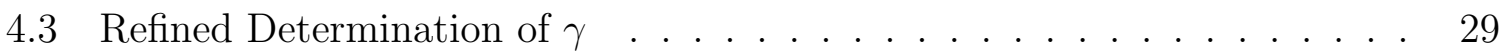

4.4 Analysis of the $B \rightarrow \pi K$ Observables . . . . . . . . . . . . . . . . . . 30

4.4.1 The $B_{d} \rightarrow \pi^{\mp} K^{ \pm}, B^{ \pm} \rightarrow \pi^{ \pm} K$ System . . . . . . . . . . . . . . . 30

4.4.2 The Charged and Neutral $B \rightarrow \pi K$ Systems . . . . . . . . . . . . 32

4.4.3 Elimination of the Second Solution for $x e^{i \Delta}$ in (3.43) . . . . . . 35

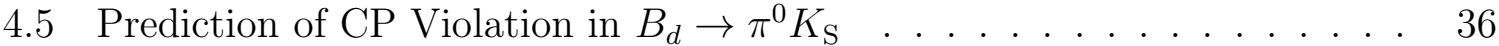

4.6 Future Avenues Offered by $B_{s}$ Decays . . . . . . . . . . . . . . . . . . . . 37

4.6.1 $B_{s} \rightarrow K^{+} K^{-} \ldots \ldots \ldots \ldots$. . . . . . . . . . 37

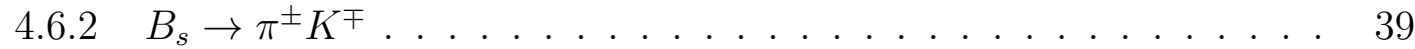

4.7 Summary . . . . . . . . . . . . . . . . . . . 40

$5 \quad$ Rare $K$ and $B$ Decays and $\varepsilon^{\prime} / \varepsilon \quad 41$

5.1 Preliminaries . . . . . . . . . . . . . . . . . . . . . . 41

$5.2 \quad K \rightarrow \pi \nu \bar{\nu} \ldots \ldots \ldots \ldots \ldots \ldots$

5.2 .1 Basic Formulae . . . . . . . . . . . . . . . . . . . . 43

5.2 .2 Numerical Results . . . . . . . . . . . . . . . . . . . . . . . . . 44

$5.3 B \rightarrow X_{s, d} \nu \bar{\nu}$ and $B_{s, d} \rightarrow \mu^{+} \mu^{-} \ldots \ldots \ldots \ldots$. . . . . . . . 46 


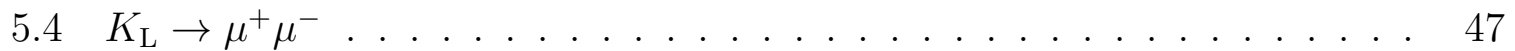

5.5 Forward-Backward Asymmetries in $b \rightarrow s \mu^{+} \mu^{-}$Modes . . . . . . . 48

5.5 .1 Basic Formulae . . . . . . . . . . . . . . . . . . . . . 48

5.5.2 Numerical Results . . . . . . . . . . . . . . . . . . . . . . . . . 49

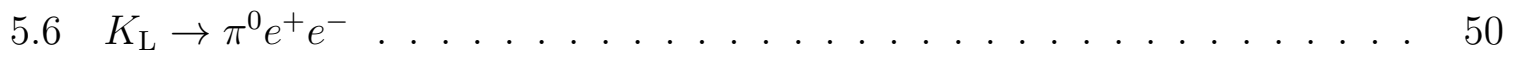

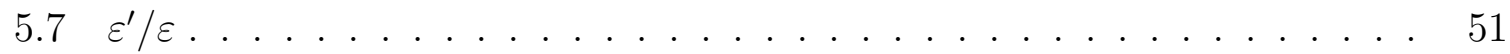

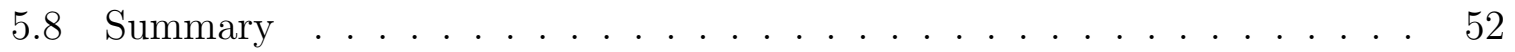

6 Other Prominent Non-Leptonic $B$-Meson Decays 53

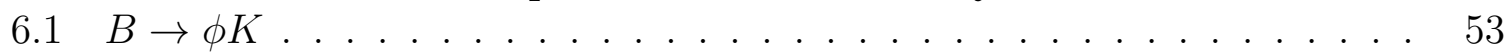

6.1 .1 Observables . . . . . . . . . . . . . . . . 53

6.1 .2 NP Analysis . . . . . . . . . . . . . . . . . . . . . 54

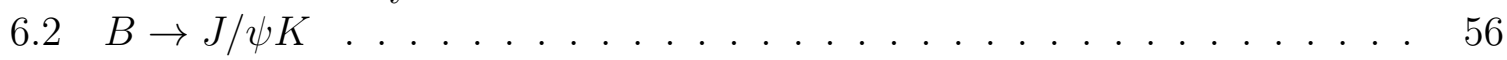

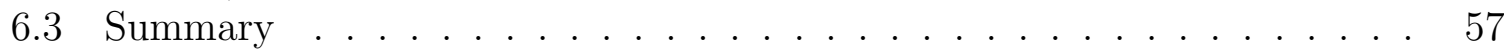

$\begin{array}{lll}7 & \text { Conclusions } & 58\end{array}$

$\begin{array}{ll}\text { A Compendium } & 61\end{array}$

A.1 The $B \rightarrow \pi \pi$ System . . . . . . . . . . . . . . . . . 61

A.2 The $B \rightarrow \pi K$ System . . . . . . . . . . . . . . . . 61

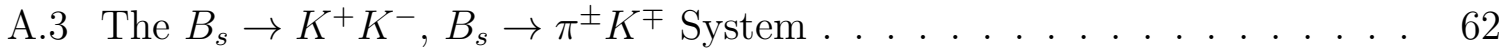

B Comment on the New Belle $B_{d} \rightarrow \pi^{+} \pi^{-}$Results 62

C Error Treatment $\quad 63$

D Colour-Suppressed EW Penguins in $B \rightarrow \pi K \quad 63$

D.1 General Structure . . . . . . . . . . . . . . . . . . . . . . 63

D.2 Generalization of the Decay Amplitudes . . . . . . . . . . . 65

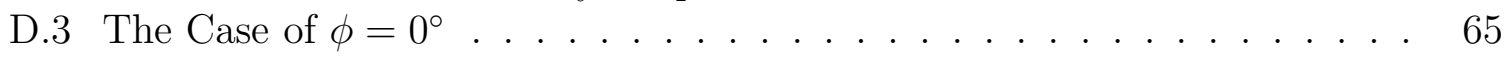

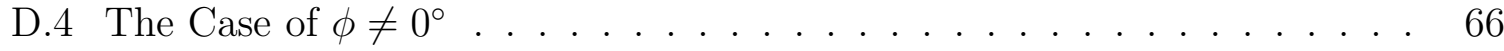




\section{Introduction}

In this decade, dedicated $B$ - and $K$-decay experiments aim at stringent tests of the flavour dynamics of the Standard Model (SM) and in particular of the KobayashiMaskawa mechanism of CP violation [1]. The central target of these studies, which will hopefully shed light on new physics (NP), is the well-known unitarity triangle (UT) of the Cabibbo-Kobayashi-Maskawa (CKM) matrix, with its three angles $\alpha, \beta$ and $\gamma$ (for detailed reviews, see $[2,3]$ ). Thanks to the SLAC and KEK $B$ factories with their detectors BaBar and Belle, respectively, mixing-induced $\mathrm{CP}$ violation is now an established effect in the $B$-meson system. The corresponding determination of $\sin \phi_{d}$ through the "golden" mode $B_{d} \rightarrow J / \psi K_{\mathrm{S}}$, where $\phi_{d}$ denotes the $B_{d}^{0}-\bar{B}_{d}^{0}$ mixing phase $\left(\phi_{d}=2 \beta\right.$ in the SM), agrees remarkably well with the CKM fits [4] that include in particular the size of the well-known "indirect" $\mathrm{CP}$ violation in $K_{\mathrm{L}} \rightarrow \pi \pi$ decays. In spite of this tremendous success of the SM, one should realize that only a handful of CP-violating decays and rare $B$ and $K$ decays have been measured and it is to be seen whether some modifications of the SM picture of flavour dynamics and CP violation will be required in the future when the data improve. For instance, the BaBar and Belle data on $\phi_{d}$ still admit two solutions with $\phi_{d} \sim 47^{\circ}$, in accordance with the SM, and $133^{\circ}$, pointing towards NP contributions. This ambiguity can be resolved by measuring the the sign of $\cos \phi_{d}[5]$, which is in progress at the $B$ factories, even though it is a challenging task [6].

While testing the SM and its possible extensions it is essential to consider simultaneously as many processes as possible. Only in this manner can the parameters of a given theory be fully determined; having them at hand, predictions for other observables can be made. In this enterprise correlations between various observables play an important rôle, as they may exclude or pinpoint a given extension of the SM even without a detailed knowledge of the parameters specific to this theory.

Interestingly, the current $B$-factory data for a number of processes indicate potential inconsistencies with the SM description of CP violation and flavour dynamics that may suggest the presence of NP contributions and/or the deficiencies in our understanding of hadron dynamics that necessarily enters the analyses of non-leptonic $B$ decays. In particular:

- The BaBar and Belle collaborations have very recently reported the observation of $B_{d} \rightarrow \pi^{0} \pi^{0}$ decays with CP-averaged branching ratios of $(2.1 \pm 0.6 \pm 0.3) \times 10^{-6}$ and $(1.7 \pm 0.6 \pm 0.2) \times 10^{-6}$, respectively $[7,8]$. These measurements represent quite a challenge for theory. For example, in a recent state-of-the-art calculation [9] within QCD factorization [10], a branching ratio that is about six times smaller is favoured, whereas the calculation of $B_{d} \rightarrow \pi^{+} \pi^{-}$points towards a branching ratio about two times larger than the current experimental average. On the other hand, the calculation of $B^{+} \rightarrow \pi^{+} \pi^{0}$ reproduces the data rather well. This " $B \rightarrow \pi \pi$ puzzle" is reflected by the following quantities:

$$
\begin{aligned}
R_{+-}^{\pi \pi} & \equiv 2\left[\frac{\mathrm{BR}\left(B^{+} \rightarrow \pi^{+} \pi^{0}\right)+\mathrm{BR}\left(B^{-} \rightarrow \pi^{-} \pi^{0}\right)}{\operatorname{BR}\left(B_{d}^{0} \rightarrow \pi^{+} \pi^{-}\right)+\operatorname{BR}\left(\bar{B}_{d}^{0} \rightarrow \pi^{+} \pi^{-}\right)}\right] \frac{\tau_{B_{d}^{0}}}{\tau_{B^{+}}}=2.12 \pm 0.37 \\
R_{00}^{\pi \pi} & \equiv 2\left[\frac{\mathrm{BR}\left(B_{d}^{0} \rightarrow \pi^{0} \pi^{0}\right)+\mathrm{BR}\left(\bar{B}_{d}^{0} \rightarrow \pi^{0} \pi^{0}\right)}{\operatorname{BR}\left(B_{d}^{0} \rightarrow \pi^{+} \pi^{-}\right)+\operatorname{BR}\left(\bar{B}_{d}^{0} \rightarrow \pi^{+} \pi^{-}\right)}\right]=0.83 \pm 0.23 .
\end{aligned}
$$


In order to calculate the numerical values, we have used $\tau_{B^{+}} / \tau_{B_{d}^{0}}=1.086 \pm 0.017$ and the most recent compilation of the Heavy Flavour Averaging Group (HFAG) [11], adding the errors in quadrature. The central values calculated within QCD factorization [9] give $R_{+-}^{\pi \pi}=1.24$ and $R_{00}^{\pi \pi}=0.07$. As was recently pointed out [12], these data indicate important non-factorizable contributions rather than NP contributions, and can be perfectly accommodated in the SM.

- In the $B \rightarrow \pi K$ system, the CLEO, BaBar and Belle collaborations have measured the following ratios of CP-averaged branching ratios [13]:

$$
\begin{aligned}
& R_{\mathrm{c}} \equiv 2\left[\frac{\mathrm{BR}\left(B^{+} \rightarrow \pi^{0} K^{+}\right)+\mathrm{BR}\left(B^{-} \rightarrow \pi^{0} K^{-}\right)}{\operatorname{BR}\left(B^{+} \rightarrow \pi^{+} K^{0}\right)+\mathrm{BR}\left(B^{-} \rightarrow \pi^{-} \bar{K}^{0}\right)}\right]=1.17 \pm 0.12 \\
& R_{\mathrm{n}} \equiv \frac{1}{2}\left[\frac{\mathrm{BR}\left(B_{d}^{0} \rightarrow \pi^{-} K^{+}\right)+\mathrm{BR}\left(\bar{B}_{d}^{0} \rightarrow \pi^{+} K^{-}\right)}{\operatorname{BR}\left(B_{d}^{0} \rightarrow \pi^{0} K^{0}\right)+\mathrm{BR}\left(\bar{B}_{d}^{0} \rightarrow \pi^{0} \bar{K}^{0}\right)}\right]=0.76 \pm 0.10,
\end{aligned}
$$

with numerical values following from [11]. As noted in [14], the pattern of $R_{\mathrm{c}}>1$ and - in particular $-R_{\mathrm{n}}<1$, which is now consistently favoured by the separate BaBar, Belle and CLEO data, is actually very puzzling. On the other hand, the quantity [15]

$$
R \equiv\left[\frac{\mathrm{BR}\left(B_{d}^{0} \rightarrow \pi^{-} K^{+}\right)+\mathrm{BR}\left(\bar{B}_{d}^{0} \rightarrow \pi^{+} K^{-}\right)}{\operatorname{BR}\left(B^{+} \rightarrow \pi^{+} K^{0}\right)+\operatorname{BR}\left(B^{-} \rightarrow \pi^{-} \bar{K}^{0}\right)}\right] \frac{\tau_{B^{+}}}{\tau_{B_{d}^{0}}}=0.91 \pm 0.07
$$

does not show any anomalous behaviour. Since $R_{\mathrm{c}}$ and $R_{\mathrm{n}}$ are affected significantly by colour-allowed electroweak (EW) penguins, whereas these topologies may only contribute to $R$ in colour-suppressed form, this " $B \rightarrow \pi K$ puzzle" may be a manifestation of NP in the EW penguin sector $[12,14,16]$, offering an attractive avenue for physics beyond the SM to enter the $B \rightarrow \pi K$ system [17]-[20].

- Another potential discrepancy with the SM expectation is indicated by the decay $B_{d} \rightarrow \phi K_{\mathrm{S}}$. Within the SM, this transition is governed by QCD penguins [21] and receives sizeable EW penguin contributions [22, 23], so that it may well be affected by NP. The current experimental status of the CP-violating $B_{d} \rightarrow \phi K_{\mathrm{S}}$ observables is given as follows [24, 25]:

$$
\begin{aligned}
& \mathcal{A}_{\mathrm{CP}}^{\mathrm{dir}}\left(B_{d} \rightarrow \phi K_{\mathrm{S}}\right)= \begin{cases}-0.38 \pm 0.37 \pm 0.12 & \text { (BaBar) } \\
+0.15 \pm 0.29 \pm 0.07 & \text { (Belle) }\end{cases} \\
& \mathcal{A}_{\mathrm{CP}}^{\text {mix }}\left(B_{d} \rightarrow \phi K_{\mathrm{S}}\right)= \begin{cases}-0.45 \pm 0.43 \pm 0.07 & \text { (BaBar) } \\
+0.96 \pm 0.50_{-0.09}^{+0.11} & \text { (Belle) },\end{cases}
\end{aligned}
$$

where we have employed the same notation for the direct and mixing-induced $\mathrm{CP}$ asymmetries $\mathcal{A}_{\mathrm{CP}}^{\mathrm{dir}}\left(B_{d} \rightarrow \phi K_{\mathrm{S}}\right)$ and $\mathcal{A}_{\mathrm{CP}}^{\mathrm{mix}}\left(B_{d} \rightarrow \phi K_{\mathrm{S}}\right)$, respectively, as in [2]. ${ }^{1}$ In the SM, the direct asymmetry is $\mathcal{O}\left(\lambda^{2}\right)$, i.e. tiny, whereas the mixing-induced $\mathrm{CP}$

\footnotetext{
${ }^{1}$ This notation may differ in signs from the one used by some authors. However, the explicit example in (3.22) and (3.23) should allow for an easy comparison of signs.
} 
asymmetry is - up to corrections of $\mathcal{O}\left(\lambda^{2}\right)$ - equal to the one of the "golden" decay $B_{d} \rightarrow J / \psi K_{\mathrm{S}}[26]-[30]$, which is measured to be

$$
\mathcal{A}_{\mathrm{CP}}^{\mathrm{mix}}\left(B_{d} \rightarrow J / \psi K_{\mathrm{S}}\right)=-0.736 \pm 0.049
$$

Consequently, we may well arrive at a discrepancy with the SM description of CP violation, although the experimental situation is of course very unclear at present.

In view of significant experimental uncertainties, none of these exciting results is conclusive at the moment, but it is legitimate and interesting to take them seriously and to search for possible origins of these "signals" for deviations from the SM expectations. As we are dealing here with non-leptonic decays, the natural question arises of whether these signals originate in the NP contributions or/and result from our insufficient understanding of the hadron dynamics. The purpose of the present paper is to develop a strategy that would allow us to address this question in a systematic manner once the experimental data on the relevant non-leptonic $B$ decays and rare $B$ and $K$ decays improve. In order to illustrate this strategy in explicit terms, we shall consider a simple extension of the SM in which NP enters dominantly through enhanced $Z^{0}$ penguins involving a $\mathrm{CP}$-violating weak phase. As we will see below, this choice is dictated by the pattern of the data on the $B \rightarrow \pi K$ observables and the great predictivity of this scenario. It was first considered in [31]-[33] to study correlations between rare $K$ decays and the ratio $\varepsilon^{\prime} / \varepsilon$ measuring direct $\mathrm{CP}$ violation in the neutral kaon system, and was generalized to rare $B$ decays in [34]. Here we extend these considerations to non-leptonic $B$-meson decays, which allows us to confront this extension of the SM with many more experimental results. Our strategy consists of three interrelated steps, and has the following logical structure:

\section{Step 1:}

Since $B \rightarrow \pi \pi$ decays and the usual analysis of the UT are only insignificantly affected by EW penguins, the $B \rightarrow \pi \pi$ system can be described as in the SM. Employing the $S U(2)$ isospin flavour symmetry of strong interactions and the information on $\gamma$ from the UT fits, we may extract the relevant hadronic parameters, and find large non-factorizable contributions, which are in particular reflected by large $\mathrm{CP}$-conserving strong phases. Having these parameters at hand, we may then also predict the direct and mixinginduced CP asymmetries of the $B_{d} \rightarrow \pi^{0} \pi^{0}$ channel. A future measurement of one of these observables allows a determination of $\gamma$.

\section{Step 2:}

If we use the $S U(3)$ flavour symmetry and plausible dynamical assumptions, we may determine the hadronic $B \rightarrow \pi K$ parameters through the $B \rightarrow \pi \pi$ analysis, and may calculate the $B \rightarrow \pi K$ observables in the SM. Interestingly, we find agreement with the pattern of the $B$-factory data for those observables where EW penguins play only a minor rôle. On the other hand, the observables receiving significant EW penguin contributions do not agree with the experimental picture, thereby suggesting NP in the EW penguin sector. Indeed, a detailed analysis shows that we may describe all the currently available data through sizeably enhanced EW penguins with a large CP-violating NP phase around $-90^{\circ}$, in the spirit of the NP scenario considered here. A crucial future test of this 
scenario will be provided by the CP-violating $B_{d} \rightarrow \pi^{0} K_{\mathrm{S}}$ observables, which we may predict. Moreover, we may obtain valuable insights into $S U(3)$-breaking effects, which support our working assumptions, and may also determine the UT angle $\gamma$, with a result in remarkable agreement with the well-known UT fits.

\section{Step 3:}

In turn, the sizeably enhanced EW penguins with their large CP-violating NP phase have important implications for rare $K$ and $B$ decays as well as $\varepsilon^{\prime} / \varepsilon$, where the new weak phase plays a particularly important rôle. Interestingly, several predictions differ significantly from the SM expectations and should easily be identified once the data improve. Similarly, we may explore specific NP patterns in other non-leptonic $B$ decays such as $B_{d} \rightarrow \phi K_{\mathrm{S}}$.

The most interesting results of this study have recently been summarized in [12]. Here we discuss the details of our analysis, present several additional results, and propose other methods that will be useful for the confrontation of the forthcoming data with the SM and the search for possible indications of NP. The outline of this paper is as follows: in Section 2, we discuss in detail our scenario of NP, using low-energy effective Hamiltonians as the starting point. In the subsequent three sections, we execute the three steps described above. In Section 3, we discuss the $B \rightarrow \pi \pi$ system in detail. We then move on to the $B \rightarrow \pi K$ system in Section 4, and explore the impact of this study on rare $K$ and $B$ decays and $\varepsilon^{\prime} / \varepsilon$, as well as the implications of the current data on rare decays on the $B \rightarrow \pi K$ observables, in Section 5. As the last element of our analysis, we investigate in Section 6 the effects of our NP scenario on the prominent $B$-meson decays of the kind $B \rightarrow \phi K$ and $B \rightarrow J / \psi K$. Finally, we summarize our conclusions in Section 7. A compendium of the most relevant formulae for $B \rightarrow \pi \pi, B_{(s)} \rightarrow \pi K$ and $B_{s} \rightarrow K^{+} K^{-}$observables and some technical details can be found in Appendices A-D.

Our paper discusses a large number of observables and it is useful already now to list them, as well as the parameters they depend on, and indicate where the explicit expressions for them can be found in our paper. We do this in Table 1, where we also present our predictions $(\mathrm{TH})$, list the input values and the present experimental values (EXP) for the observables in question. It should be emphasized that, within our approach, the hadronic $B_{(s)} \rightarrow \pi K$ and $B_{s} \rightarrow K^{+} K^{-}$parameters can be calculated in terms of the parameters of the $B \rightarrow \pi \pi$ system. The relevant formulae are given in (4.17), (4.18), (4.20) and (4.21). A compendium of the formulae for all observables of Table 1 is given in Appendix A.

\section{A Simple Scenario for New Physics}

\subsection{General Structure}

The scenario of NP with the dominant $Z^{0}$-penguin contributions presented here was first considered in [31]-[33], where correlations between rare $K$ decays and $\varepsilon^{\prime} / \varepsilon$ were studied. It was generalized to rare $B$ decays in [34]. The new feature of our analysis is a simultaneous study of rare $K$ and $B$ decays, in addition to non-leptonic $B$ decays, in 


\begin{tabular}{|c|c|c|c|c|}
\hline Quantity & Eq. & Parameters & $\mathrm{TH}$ & EXP \\
\hline$R_{+-}^{\pi \pi}$ & $(3.20)$ & $x, \Delta, d, \theta$ & input & $2.12 \pm 0.37$ \\
\hline$R_{00}^{\pi \pi}$ & $(3.21)$ & $x, \Delta, d, \theta$ & input & $0.83 \pm 0.23$ \\
\hline $\mathcal{A}_{\mathrm{CP}}^{\mathrm{dir}}\left(B_{d} \rightarrow \pi^{+} \pi^{-}\right)$ & $(3.25)$ & $d, \theta$ & input & $-0.38 \pm 0.16$ \\
\hline $\mathcal{A}_{\mathrm{CP}}^{\operatorname{mix}}\left(B_{d} \rightarrow \pi^{+} \pi^{-}\right)$ & $(3.26)$ & $d, \theta, \phi_{d}$ & input & $0.58 \pm 0.20$ \\
\hline $\mathcal{A}_{\mathrm{CP}}^{\mathrm{dir}}\left(B_{d} \rightarrow \pi^{0} \pi^{0}\right)$ & $(3.69)$ & $x, \Delta, d, \theta$ & $-0.41_{-0.17}^{+0.35}$ & - \\
\hline $\mathcal{A}_{\mathrm{CP}}^{\operatorname{mix}}\left(B_{d} \rightarrow \pi^{0} \pi^{0}\right)$ & $(3.70)$ & $x, \Delta, d, \theta, \phi_{d}$ & $-0.55_{-0.45}^{+0.43}$ & - \\
\hline$R$ & $(4.32)$ & $r, \delta$ & $0.943_{-0.026}^{+0.033}$ & $0.91 \pm 0.07$ \\
\hline$R_{\mathrm{c}}$ & $(4.36)$ & $r_{\mathrm{c}}, \delta_{\mathrm{c}}, q, \omega, \phi$ & $1.00_{-0.08}^{+0.12}$ & $1.17 \pm 0.12$ \\
\hline$R_{\mathrm{n}}$ & $(4.37)$ & $r, \delta, r_{\mathrm{c}}, \delta_{\mathrm{c}}, q, \omega, \phi, \rho_{\mathrm{n}}, \theta_{\mathrm{n}}$ & $0.82_{-0.11}^{+0.12}$ & $0.76 \pm 0.10$ \\
\hline $\mathcal{A}_{\mathrm{CP}}^{\mathrm{dir}}\left(B_{d} \rightarrow \pi^{\mp} K^{ \pm}\right)$ & $(4.34)$ & $r, \delta$ & $0.140_{-0.087}^{+0.139}$ & $0.095 \pm 0.028$ \\
\hline $\mathcal{A}_{\mathrm{CP}}^{\mathrm{dir}}\left(B^{ \pm} \rightarrow \pi^{ \pm} K\right)$ & $(4.11)$ & $\rho_{\mathrm{c}}, \theta_{\mathrm{c}}$ & $\sim 0$ & $-0.02 \pm 0.06$ \\
\hline $\mathcal{A}_{\mathrm{CP}}^{\mathrm{dir}}\left(B^{ \pm} \rightarrow \pi^{0} K^{ \pm}\right)$ & $(4.50)$ & $r_{\mathrm{c}}, \delta_{\mathrm{c}}, q, \omega, \phi$ & $0.03_{-0.24}^{+0.32}$ & $0.00 \pm 0.07$ \\
\hline $\mathcal{A}_{\mathrm{CP}}^{\mathrm{dir}}\left(B_{d} \rightarrow \pi^{0} K_{\mathrm{S}}\right)$ & $(4.59)$ & $r, \delta, r_{\mathrm{c}}, \delta_{\mathrm{c}}, q, \omega, \phi, \rho_{\mathrm{n}}, \theta_{\mathrm{n}}$ & $0.08_{-0.22}^{+0.18}$ & $0.40_{-0.30}^{+0.29}$ \\
\hline $\mathcal{A}_{\mathrm{CP}}^{\operatorname{mix}}\left(B_{d} \rightarrow \pi^{0} K_{\mathrm{S}}\right)$ & $(4.60)$ & $r, \delta, r_{\mathrm{c}}, \delta_{\mathrm{c}}, q, \omega, \phi, \rho_{\mathrm{n}}, \theta_{\mathrm{n}}, \phi_{d}$ & $-0.98_{-0.02}^{+0.05}$ & $-0.48_{-0.40}^{+0.48}$ \\
\hline $\mathcal{A}_{\mathrm{CP}}^{\mathrm{dir}}\left(B_{s} \rightarrow K^{+} K^{-}\right)$ & $(4.70)$ & $d, \theta$ & $0.14_{-0.09}^{+0.14}$ & - \\
\hline $\mathcal{A}_{\mathrm{CP}}^{\operatorname{mix}}\left(B_{s} \rightarrow K^{+} K^{-}\right)$ & $(4.71)$ & $d, \theta, \phi_{s}$ & $-0.18_{-0.07}^{+0.08}$ & - \\
\hline $\mathcal{A}_{\mathrm{CP}}^{\operatorname{dir}}\left(B_{s} \rightarrow \pi^{ \pm} K^{\mp}\right)$ & $(4.83)$ & $r, \delta$ & $-0.38_{-0.16}^{+0.16}$ & - \\
\hline
\end{tabular}

Table 1: A guide to master formulae. We suppress the dependence on $\gamma$ as it enters all quantities. For those quantities that depend on the EW penguin parameters $q$ and $\phi$, the rare decays constraints as discussed in Section 5 have been employed in the calculation of our theoretical values $(\mathrm{TH})$.

particular $B \rightarrow \pi \pi, \pi K$ modes.

It should be noted that in [31]-[34] model-independent analyses and studies within particular supersymmetric scenarios were presented. As we will see below, in the present analysis, we determine the size of the enhancement of the $Z^{0}$-penguin function $C$ and the magnitude of its complex phase by the $B \rightarrow \pi K$ data and, consequently, our predictions are more specific than was possible in these papers. In what follows, we will recall the basic ingredients of this scenario in a notation suitable to our analysis.

In order to discuss the non-leptonic $B$-meson decays and rare $K$ and $B$ decays in a particular extension of the SM, and to investigate correlations between the NP contributions to different observables, it is essential to formulate the theory with the help of an effective Hamiltonian. The effective weak Hamiltonian for $\Delta F=1$ decays with $F=S, B$ is generally given as follows [35]:

$$
\mathcal{H}_{\mathrm{eff}}=\frac{G_{\mathrm{F}}}{\sqrt{2}} \sum_{i} V_{\mathrm{CKM}}^{i} C_{i}(\mu) Q_{i},
$$

where $G_{\mathrm{F}}$ is the Fermi constant and $V_{\mathrm{CKM}}^{i}$ the relevant CKM factors. Next, the $Q_{i}$ are local operators, which govern the decays in question, and the $C_{i}(\mu)$ are the corre- 
sponding Wilson coefficients, which describe the strength with which a given operator enters the Hamiltonian. The latter coefficients can be calculated in renormalizationgroup-improved perturbation theory and carry, in particular, the information about the physics contributions at scales higher than $\mu$, which is usually chosen to be $\mathcal{O}\left(m_{b}\right)$ and $\mathcal{O}((1-2) \mathrm{GeV})$ for $B$ decays and $K$ decays, respectively. Thus, the $C_{i}$ include the topquark contributions and contributions from other heavy particles if extensions of the SM are considered. Consequently, the $C_{i}(\mu)$ generally depend on the top-quark mass $m_{t}$ and also on the masses and couplings of the new particles. This dependence can be found by evaluating box and penguin diagrams with $W-, Z$-, top- and new-particle exchanges and properly including short-distance QCD effects.

The amplitude for the decay of a given meson $M=K, B, \ldots$ into a final state $F=$ $\pi \pi, \pi K, \pi \nu \bar{\nu}, \ldots$ is then simply given by

$$
A(M \rightarrow F)=\left\langle F\left|\mathcal{H}_{\mathrm{eff}}\right| M\right\rangle=\frac{G_{\mathrm{F}}}{\sqrt{2}} \sum_{i} V_{\mathrm{CKM}}^{i} C_{i}(\mu)\left\langle F\left|Q_{i}(\mu)\right| M\right\rangle,
$$

where the $\left\langle F\left|Q_{i}(\mu)\right| M\right\rangle$ are the hadronic matrix elements of the operators $Q_{i}$ between $M$ and $F$, evaluated at the renormalization scale $\mu$. As demonstrated in [36, 37], the formula (2.2) can be cast into the following useful expression:

$$
A(\text { Decay })=P_{0}(\text { Decay })+\sum_{r} P_{r}(\text { Decay }) F_{r}(v) .
$$

To this end, we choose $\mu=\mu_{0}=\mathcal{O}\left(M_{W}, m_{t}\right)$ in (2.2) and rewrite the $C_{i}\left(\mu_{0}\right)$ as linear combinations of the so-called master functions $F_{r}(v)$, which result from various penguin and box diagrams with heavy particle exchanges and $v$ denoting collectively the parameters of a given model. In the SM, the functions $F_{r}(v)$ reduce to the Inami-Lim functions [38], with $v=m_{t}^{2} / M_{W}^{2}$. The term $P_{0}$ summarizes the contributions stemming from light internal quarks, such as the charm and up quarks, and the sum incorporates the remaining contributions. The general properties of the $P_{0}, P_{r}$ and $F_{r}$ have recently been discussed at length in [39]. We recall here only the following features:

- $F_{r}(v)$ are process-independent, universal functions that depend on the particular model considered. NP enters the decay amplitudes only through these functions.

- $P_{0}$ and $P_{r}$ are process-dependent quantities. In particular, they depend on the hadronic matrix elements of the operators $Q_{i}{ }^{2}$

In the models with "minimal flavour violation" (MFV), as defined in [39, 40], the set of the $F_{r}(v)$ consists of seven functions

$$
S(v), X(v), Y(v), Z(v), E(v), D^{\prime}(v), E^{\prime}(v),
$$

which are discussed in detail in [39]. The important property of the functions in (2.4) is that they are real-valued, so that the CP-violating effects are governed entirely by the complex CKM phase hiding in the parameters $P_{r}$. Other definitions of MFV can be found in $[41,42]$.

\footnotetext{
${ }^{2}$ These quantities should not be confused with the penguin parameters in Sections 3 and 4 .
} 


\subsection{Going Beyond MFV}

In the present paper, we would like to investigate a scenario along the lines of [31]-[34], which goes beyond the usual MFV framework. In particular, we consider the presence of a single weak complex phase in the functions $F_{r}(v)$, leading to additional CP-violating effects that are not present in models with MFV. In order to make the discussion as simple as possible, we will choose the functions $S, E, D^{\prime}$ and $E^{\prime}$ to be real-valued, as in the MFV case. Moreover, we will assume that the functions $E, D^{\prime}$ and $E^{\prime}$ are not affected significantly by NP contributions, so that $D^{\prime}$ and $E^{\prime}$, which result from magnetic penguin diagrams, describe with their SM values automatically the $B \rightarrow X_{s} \gamma$ decay. The function $E$ resulting from QCD penguins and entering the Wilson coefficients of penguin operators at scales $\mathcal{O}\left(M_{W}\right)$ is phenomenologically not very relevant and can safely be set to its SM value. The point is that the contributions of QCD penguins to essentially all decays are dominated by renormalization-group effects for scales below $M_{W}$ and are included in the coefficient $P_{0}$ in (2.3).

Concerning the $\Delta F=2$ box function $S(v)$, it enters only the CP violation parameter $\varepsilon_{K}$ and the $B_{d, s}^{0}-\bar{B}_{d, s}^{0}$ mass differences $\Delta M_{d, s}$. Being real, it does not introduce any new complex phases in the standard analysis of the UT that is based on $\left|V_{c b}\right|,\left|V_{u b} / V_{c b}\right|, \varepsilon_{K}$ and $\Delta M_{d, s}$. On the other hand, the enhanced function $C$ and its large complex phase can, in principle, affect the analysis of the UT through the double $Z^{0}$-penguin contributions to $\varepsilon_{K}$ and $\Delta M_{s, d}$. However, we have checked that these effects are at the few-percent level. In particular, the phases of the $B_{d, s}^{0}-\bar{B}_{d, s}^{0}$ mixing amplitudes are essentially unchanged.

Next the decay amplitude of the "golden" mode $B_{d} \rightarrow J / \psi K_{\mathrm{S}}$ is only insignificantly, i.e. at the level of the current experimental and theoretical uncertainties, affected by the $\mathrm{NP}$ in the EW penguin sector in our scenario. Consequently, as discussed in detail in Subsection 6.2, we may still convert - to a good approximation - the mixing-induced CP asymmetry $\mathcal{A}_{\mathrm{CP}}^{\mathrm{mix}}\left(B_{d} \rightarrow J / \psi K_{\mathrm{S}}\right)$ into the angle $\beta$ of the UT, as in the SM. In particular, a "universal unitarity triangle" [40] can also be constructed in our NP scenario by using only $\left|V_{c b}\right|, \Delta M_{d} / \Delta M_{s}$ and $\sin 2 \beta$. This simplifies our phenomenological analysis significantly, since the determination of the CKM parameters can be separated from the study of the hadronic and NP effects in non-leptonic $B$ decays and rare decays. However, as advertised in Step 2 of our approach, one can also determine $\gamma$ by complementing the $B \rightarrow \pi \pi$ system with those $B \rightarrow \pi K$ modes that are insensitive to the NP effects discussed here, i.e. to EW penguin contributions, and subsequently compare the result with the one obtained by using the conventional UT fits. Following these lines and using the experimental information on the side $R_{b}$ of the UT, we may also determine the remaining two angles $\alpha$ and $\beta$ in an alternative manner, and eventually arrive at a remarkably consistent overall picture.

Most interesting for us are the functions $X, Y$ and $Z$, which are given by

$$
X(v)=C(v)+B^{\nu \bar{\nu}}(v), \quad Y(v)=C(v)+B^{\mu \bar{\mu}}(v), \quad Z(v)=C(v)+\frac{1}{4} D(v),
$$

with $C(v), D(v), B^{\nu \bar{\nu}}(v)$ and $B^{\mu \bar{\mu}}(v)$ resulting from $Z^{0}$-penguin diagrams, $\gamma$-penguin diagrams and $\Delta F=1$ box diagrams with $\nu \bar{\nu}$ and $\mu \bar{\mu}$, respectively. Moreover, explicit calculations indicate that in the case of $\Delta F=1$ box diagrams with $u \bar{u}$ and $d \bar{d}$ in the 
final state we have, to an excellent approximation:

$$
B^{u \bar{u}}(v)=B^{\nu \bar{\nu}}(v), \quad B^{d \bar{d}}(v)=B^{\mu \bar{\mu}}(v)
$$

so that the functions $X$ and $Y$ in (2.5) are valid for non-leptonic decays as well. Similarly, it is found that the NP contributions to these $\Delta F=1$ box diagrams and to $D$ are rather small, so that we may use for them, in the following analysis, their SM values $\left(m_{t}=167 \mathrm{GeV}\right)$, which are given by

$$
B^{u \bar{u}}(v)=B^{\nu \bar{\nu}}(v)=0.73, \quad B^{d \bar{d}}(v)=B^{\mu \bar{\mu}}(v)=0.18, \quad D(v)=-0.48
$$

As a consequence of these approximations, the dominant NP effects come from the $Z^{0}$ penguin function $C(v)$. In the standard MFV scenarios, also the one considered in [16], the function $C(v)$ is real-valued. Here it contains a weak phase $\theta_{C}$. Consequently, the functions $X, Y$ and $Z$ are now given as follows:

$$
X(v)=|C(v)| e^{i \theta_{C}}+0.73, \quad Y(v)=|C(v)| e^{i \theta_{C}}+0.18, \quad Z(v)=|C(v)| e^{i \theta_{C}}-0.12 .
$$

While our analysis does not rely on a particular model with the properties specified above, concrete models with enhanced $\mathrm{CP}$-violating $Z^{0}$-mediated FCNC couplings generated either at the one-loop level or even at the tree level have been discussed in the literature. They are reviewed in [31]-[34], in particular in the last of these papers; see also [43]. Also models with $Z^{\prime}$-mediated FCNCs could be put in this class, provided their contributions can effectively be absorbed in the function $C(v)$. For a recent analysis, see [44].

\subsection{Relation Between Rare Decays and $B \rightarrow \pi K$ Modes}

As already discussed in [16], the connection between the rare decays and the $B \rightarrow \pi K$ system is established by relating the function $C$ to the EW penguin parameter $q$ by means of a renormalization-group analysis. In the case of a complex $C$, the relation given in [16] is generalized as follows:

$$
|C(v)| e^{i \theta_{C}}=2.35 \bar{q} e^{i \phi}-0.82, \quad \bar{q}=q\left[\frac{\left|V_{u b} / V_{c b}\right|}{0.086}\right]
$$

where $q$ and $\phi$ characterize the EW penguin sector and enter the parametrization of the $B \rightarrow \pi K$ decays given in Section 4 . The numerical coefficients in (2.9) correspond to $\alpha_{s}\left(M_{Z}\right)=0.119$ and depend very weakly on its value.

In order to define $q$ and $\phi$, we recall formula (43) of [13] for the ratio of the EW penguin amplitude $P_{\mathrm{EW}}^{\prime}$ to the tree contributions in $B \rightarrow \pi K$ decays (see also Section 4 ), yielding

$$
\left|\frac{P_{\mathrm{EW}}^{\prime}}{T^{\prime}+C^{\prime}}\right| e^{i\left(\delta_{\mathrm{EW}}^{\prime}-\delta_{T^{\prime}+C^{\prime}}\right)}=-\frac{3}{2} \frac{1}{\lambda\left|V_{u b} / V_{c b}\right|}\left[\frac{C_{9}\left(\mu_{b}\right)+C_{10}\left(\mu_{b}\right) \tilde{\xi}}{C_{1}^{\prime}\left(\mu_{b}\right)+C_{2}^{\prime}\left(\mu_{b}\right) \tilde{\xi}}\right],
$$

where $\tilde{\xi}=1$ in the $S U(3)$ symmetry limit $[13,45]$. Here $C_{9}\left(\mu_{b}\right)$ and $C_{10}\left(\mu_{b}\right)\left(\mu_{b}=\mathcal{O}\left(m_{b}\right)\right)$ are the Wilson coefficients of the $(V-A) \otimes(V-A)$ EW penguin operators $Q_{9}$ and $Q_{10}$, 
respectively, which enter the effective Hamiltonian for $\Delta B=1$ non-leptonic decays [35], and the coefficients $C_{1,2}^{\prime}\left(\mu_{b}\right)$ are given as follows [46]:

$$
C_{1}^{\prime}\left(\mu_{b}\right)=C_{1}\left(\mu_{b}\right)+\frac{3}{2} C_{9}\left(\mu_{b}\right), \quad C_{2}^{\prime}\left(\mu_{b}\right)=C_{2}\left(\mu_{b}\right)+\frac{3}{2} C_{10}\left(\mu_{b}\right)
$$

with $C_{1,2}$ being the Wilson coefficients of the current-current operators $Q_{1,2}$. As $C_{9,10}=$ $\mathcal{O}(\alpha)$ and $C_{1,2}=\mathcal{O}(1)$, we will approximate $C_{1,2}^{\prime}$ by $C_{1,2}$. Moreover, we will set $\tilde{\xi}=1$ in front of $C_{10}$. This approximation is justified by the fact that $C_{10}\left(M_{W}\right)=0$ and consequently $C_{10}\left(\mu_{b}\right)$ is significantly smaller than $C_{9}\left(\mu_{b}\right)$. Next, in the NP scenario outlined above, we may write

$$
C_{9}\left(\mu_{b}\right)+C_{10}\left(\mu_{b}\right)=h+g C(v) e^{i \theta_{C}} \equiv-A_{\mathrm{EW}} e^{i \phi}
$$

where $h$ and $g$ are calculable by means of the two-loop renormalization-group formula given in [47]. The minus sign in (2.12) causes the parameter $A_{\mathrm{EW}}$ to be positive in the SM. Writing then $\tilde{\xi}=\xi e^{i \tau}, \tau$ being a strong phase, we obtain

$$
C_{1}\left(\mu_{b}\right)+C_{2}\left(\mu_{b}\right) \xi e^{i \tau} \equiv B_{\mathrm{CC}} e^{-i \omega}
$$

where it was convenient to introduce a strong phase $\omega[13]$, which vanishes in the $S U(3)$ limit $[45,48]$. Consequently, (2.10) yields the following simple expression:

$$
\left|\frac{P_{\mathrm{EW}}^{\prime}}{T^{\prime}+C^{\prime}}\right| e^{i\left(\delta_{\mathrm{EW}}^{\prime}-\delta_{T^{\prime}+C^{\prime}}\right)}=q e^{i \phi} e^{i \omega}, \quad q=\frac{3}{2} \frac{1}{\lambda \mid V_{u b} / V_{c b}} \mid \frac{A_{\mathrm{EW}}}{B_{\mathrm{CC}}},
$$

which we will use for the parametrization of the $B \rightarrow \pi K$ decays in Section 4 .

As far as the rare decays considered in Section 5 are concerned, they can be directly expressed in terms of the $X, Y$ and $Z$ functions introduced in (2.8). Consequently, in order to discuss the correlation between the $B \rightarrow \pi K$ decays and the rare decays, it is useful to express them in terms of $(\bar{q}, \phi)$ by inserting (2.9) into (2.8). The corresponding expressions are given in Section 5.

\subsection{CKM Parameters}

Concerning the CKM parameters, we will use the Wolfenstein parametrization [49], generalized to include higher orders in $\lambda \equiv\left|V_{u s}\right|[50]$. Writing then

$$
V_{t d}=A R_{t} \lambda^{3} e^{-i \beta}, \quad V_{t s}=-\left|V_{t s}\right| e^{-i \beta_{s}}
$$

with $\tan \beta_{s} \approx-\lambda^{2} \bar{\eta}$, we have

$$
\lambda_{t} \equiv V_{t s}^{*} V_{t d}=-\tilde{r} A^{2} \lambda^{5} R_{t} e^{-i \beta} e^{i \beta_{s}} \quad \text { with } \quad \tilde{r}=\left|\frac{V_{t s}}{V_{c b}}\right| \approx 0.98,
$$

and

$$
\tilde{\lambda}_{t}^{(d)} \equiv V_{t b}^{*} V_{t d}=A \lambda^{3} R_{t} e^{-i \beta}, \quad \tilde{\lambda}_{t}^{(s)} \equiv V_{t b}^{*} V_{t s}=-\tilde{r} A \lambda^{2} e^{-i \beta_{s}}
$$


where $R_{t}$ is one of the sides in the UT, and $A \equiv\left|V_{c b}\right| / \lambda^{2}$ is the usual Wolfenstein parameter. Moreover, we have the following useful relations for the two UT sides $R_{t}$ and $R_{b}$ and the ratio $\left|V_{u b} / V_{c b}\right|[3]$ :

$$
R_{t}=\frac{\sin \gamma}{\sin (\beta+\gamma)}, \quad R_{b} \equiv\left(1-\frac{\lambda^{2}}{2}\right) \frac{1}{\lambda}\left|\frac{V_{u b}}{V_{c b}}\right|, \quad\left|\frac{V_{u b}}{V_{c b}}\right|=\left(\frac{\lambda}{1-\lambda^{2} / 2}\right) \frac{\sin \beta}{\sin (\beta+\gamma)}
$$

For our numerical analyses, we will use

$$
\begin{gathered}
\lambda=0.2240 \pm 0.0036, \quad A=0.83 \pm 0.02, \quad\left|\frac{V_{u b}}{V_{c b}}\right|=0.086 \pm 0.008, \quad R_{b}=0.37 \pm 0.04 \\
\beta=(23.5 \pm 2.0)^{\circ}, \quad \beta_{s}=-1^{\circ} .
\end{gathered}
$$

\subsection{Summary}

Before turning to the $B \rightarrow \pi \pi$ system, let us summarize the main results of this section:

- The NP scenario considered here involves two parameters, $C(v)$ and $\theta_{C}$.

- The relevant EW penguin parameters for the $B \rightarrow \pi K$ decays are $q, \phi$ and $\omega$.

- The parameter sets $\left(C, \theta_{C}\right)$ and $(q, \phi)$ are related through $(2.9)$, which allows us to investigate the correlations between rare decays and non-leptonic $B$ decays.

\section{The $B \rightarrow \pi \pi$ System}

In the literature, $B \rightarrow \pi \pi$ decays are usually considered in the context of the determination of the UT angle $\alpha[51,52]$. Here we shall use these modes from a very different point of view, which is inspired by the analyses performed in [53]-[56]. Very recently, the decay $B_{d} \rightarrow \pi^{+} \pi^{-}$was also discussed in [57] in the context of bounds on $\gamma$ and the

UT. However, these approaches differ significantly from the one presented in [12] and here. In particular, the channels involving neutral pions are not discussed at all in [57].

\subsection{Basic Formulae}

In order to make our analysis more transparent, we shall first neglect the EW penguin contributions to the $B \rightarrow \pi \pi$ decays, which play a very minor rôle [58, 59]; using the isospin flavour symmetry of strong interactions, we include these contributions in Subsection 3.6, following $[13,60]$. We may then write the $B \rightarrow \pi \pi$ amplitudes as

$$
\begin{aligned}
\sqrt{2} A\left(B^{+} \rightarrow \pi^{+} \pi^{0}\right) & =-[\tilde{T}+\tilde{C}]=-[T+C] \\
A\left(B_{d}^{0} \rightarrow \pi^{+} \pi^{-}\right) & =-[\tilde{T}+P] \\
\sqrt{2} A\left(B_{d}^{0} \rightarrow \pi^{0} \pi^{0}\right) & =-[\tilde{C}-P],
\end{aligned}
$$


with

$$
\begin{aligned}
P & =\lambda^{3} A\left(\mathcal{P}_{t}-\mathcal{P}_{c}\right) \equiv \lambda^{3} A \mathcal{P}_{t c} \\
\tilde{T} & =\lambda^{3} A R_{b} e^{i \gamma}\left[\mathcal{T}-\left(\mathcal{P}_{t u}-\mathcal{E}\right)\right] \\
\tilde{C} & =\lambda^{3} A R_{b} e^{i \gamma}\left[\mathcal{C}+\left(\mathcal{P}_{t u}-\mathcal{E}\right)\right] .
\end{aligned}
$$

The parameters of the CKM matrix entering these formulae have been defined in Subsection 2.4, whereas the $\mathcal{P}_{q}$ describe the strong amplitudes of QCD penguins with internal $q$-quark exchanges $(q \in\{t, c, u\}),{ }^{3}$ including annihilation and exchange penguins; $\mathcal{T}$ and $\mathcal{C}$ are the strong amplitudes of colour-allowed and colour-suppressed tree-diagram-like topologies, respectively; and $\mathcal{E}$ denotes the strong amplitude of an exchange amplitude. In the usual notation employed in the literature (see, for instance, [58]), the colourallowed and colour-suppressed tree-diagram-like amplitudes

$$
\begin{aligned}
& T=\lambda^{3} A R_{b} e^{i \gamma} \mathcal{T} \\
& C=\lambda^{3} A R_{b} e^{i \gamma} \mathcal{C}
\end{aligned}
$$

appear; they differ from the $\tilde{T}$ and $\tilde{C}$ amplitudes through the $\left(\mathcal{P}_{t u}-\mathcal{E}\right)$ pieces, which may actually play an important rôle, as was emphasized in [64]. However, we observe that these terms cancel in

$$
\tilde{T}+\tilde{C}=T+C
$$

and that the amplitudes in (3.1)-(3.3) satisfy the well-known isospin relation [51]

$$
\sqrt{2} A\left(B^{+} \rightarrow \pi^{+} \pi^{0}\right)=A\left(B_{d}^{0} \rightarrow \pi^{+} \pi^{-}\right)+\sqrt{2} A\left(B_{d}^{0} \rightarrow \pi^{0} \pi^{0}\right) .
$$

It is convenient to rewrite these amplitudes as

$$
\begin{aligned}
\sqrt{2} A\left(B^{+} \rightarrow \pi^{+} \pi^{0}\right) & =-|\tilde{T}| e^{i \delta_{\tilde{T}}} e^{i \gamma}\left[1+x e^{i \Delta}\right] \\
A\left(B_{d}^{0} \rightarrow \pi^{+} \pi^{-}\right) & =-|\tilde{T}| e^{i \delta_{\tilde{T}}}\left[e^{i \gamma}-d e^{i \theta}\right] \\
\sqrt{2} A\left(B_{d}^{0} \rightarrow \pi^{0} \pi^{0}\right) & =|P| e^{i \delta_{P}}\left[1+\frac{x}{d} e^{i \gamma} e^{i(\Delta-\theta)}\right],
\end{aligned}
$$

with

$$
\begin{gathered}
x e^{i \Delta} \equiv \frac{\tilde{C}}{\tilde{T}}=\left|\frac{\tilde{C}}{\tilde{T}}\right| e^{i\left(\delta_{\tilde{C}}-\delta_{\tilde{T}}\right)}=\frac{\mathcal{C}+\left(\mathcal{P}_{t u}-\mathcal{E}\right)}{\mathcal{T}-\left(\mathcal{P}_{t u}-\mathcal{E}\right)} \\
d e^{i \theta} \equiv-\frac{P}{\tilde{T}} e^{i \gamma}=-\left|\frac{P}{\tilde{T}}\right| e^{i\left(\delta_{P}-\delta_{\tilde{T}}\right)}=-\frac{1}{R_{b}}\left[\frac{\mathcal{P}_{t c}}{\mathcal{T}-\left(\mathcal{P}_{t u}-\mathcal{E}\right)}\right],
\end{gathered}
$$

where the latter parameter was already introduced in [53], and $\delta_{\tilde{T}}$ and $\delta_{P}$ denote the CP-conserving strong phases of the amplitudes $\tilde{T}$ and $P$, respectively. If we now consider

\footnotetext{
${ }^{3}$ Strictly speaking in the case of $\mathcal{P}_{t}$ this standard terminology could be misleading, as the top-quark effects in amplitudes for $B$ decays are only present in the relevant Wilson coefficients - also absorbed in $\mathcal{P}_{q}$ - and not in hadronic matrix elements. For relations between the language used here to that of the operator product expansion, see [61]-[63].
} 
the corresponding CP-averaged decay-amplitude squares, which are generically defined through

$$
\left\langle|A|^{2}\right\rangle \equiv \frac{1}{2}\left[|A(B \rightarrow f)|^{2}+|A(\bar{B} \rightarrow \bar{f})|^{2}\right]
$$

we obtain

$$
\begin{gathered}
\left\langle\left|A\left(B^{ \pm} \rightarrow \pi^{ \pm} \pi^{0}\right)\right|^{2}\right\rangle=\frac{|\tilde{T}|^{2}}{2}\left[1+2 x \cos \Delta+x^{2}\right] \\
\left\langle\left|A\left(B_{d} \rightarrow \pi^{+} \pi^{-}\right)\right|^{2}\right\rangle=|\tilde{T}|^{2}\left[1-2 d \cos \theta \cos \gamma+d^{2}\right] \\
\left\langle\left|A\left(B_{d} \rightarrow \pi^{0} \pi^{0}\right)\right|^{2}\right\rangle=\frac{|\tilde{P}|^{2}}{2}\left[1+2\left(\frac{x}{d}\right) \cos (\Delta-\theta) \cos \gamma+\left(\frac{x}{d}\right)^{2}\right] .
\end{gathered}
$$

These quantities provide two independent ratios of $\mathrm{CP}$-averaged branching ratios, which we may choose as those introduced in (1.1) and (1.2). Using (3.17)-(3.19), we obtain

$$
\begin{gathered}
R_{+-}^{\pi \pi}=\frac{1+2 x \cos \Delta+x^{2}}{1-2 d \cos \theta \cos \gamma+d^{2}} \\
R_{00}^{\pi \pi}=\frac{d^{2}+2 d x \cos (\Delta-\theta) \cos \gamma+x^{2}}{1-2 d \cos \theta \cos \gamma+d^{2}} .
\end{gathered}
$$

In addition to $R_{+-}^{\pi \pi}$ and $R_{00}^{\pi \pi}$, also the time-dependent $\mathrm{CP}$ asymmetries of the decays $B_{d} \rightarrow \pi^{+} \pi^{-}$and $B_{d} \rightarrow \pi^{0} \pi^{0}$ provide valuable information. Thanks to the efforts at the $B$ factories, experimental results for the former channel are already available, which is a decay into a CP-even final state, exhibiting the following rate asymmetry [2]:

$$
\begin{aligned}
& \frac{\Gamma\left(B_{d}^{0}(t) \rightarrow \pi^{+} \pi^{-}\right)-\Gamma\left(\bar{B}_{d}^{0}(t) \rightarrow \pi^{+} \pi^{-}\right)}{\Gamma\left(B_{d}^{0}(t) \rightarrow \pi^{+} \pi^{-}\right)+\Gamma\left(\bar{B}_{d}^{0}(t) \rightarrow \pi^{+} \pi^{-}\right)} \\
& \quad=\mathcal{A}_{\mathrm{CP}}^{\text {dir }}\left(B_{d} \rightarrow \pi^{+} \pi^{-}\right) \cos \left(\Delta M_{d} t\right)+\mathcal{A}_{\mathrm{CP}}^{\operatorname{mix}}\left(B_{d} \rightarrow \pi^{+} \pi^{-}\right) \sin \left(\Delta M_{d} t\right) .
\end{aligned}
$$

Here, $\Delta M_{d}>0$ is the mass difference of the mass eigenstates of the $B_{d}$-meson system, while the $\mathrm{CP}$-violating observables

$$
\mathcal{A}_{\mathrm{CP}}^{\mathrm{dir}}\left(B_{d} \rightarrow \pi^{+} \pi^{-}\right) \equiv \frac{1-\left|\xi_{\pi^{+} \pi^{-}}^{(d)}\right|^{2}}{1+\left|\xi_{\pi^{+} \pi^{-}}^{(d)}\right|^{2}} \quad \text { and } \quad \mathcal{A}_{\mathrm{CP}}^{\operatorname{mix}}\left(B_{d} \rightarrow \pi^{+} \pi^{-}\right) \equiv \frac{2 \operatorname{Im} \xi_{\pi^{+} \pi^{-}}^{(d)}}{1+\left|\xi_{\pi^{+} \pi^{-}}^{(d)}\right|^{2}}
$$

describe "direct" and "mixing-induced" CP violation, respectively, and are governed by

$$
\xi_{\pi^{+} \pi^{-}}^{(d)}=-e^{-i \phi_{d}}\left[\frac{e^{-i \gamma}-d e^{i \theta}}{e^{+i \gamma}-d e^{i \theta}}\right] .
$$

The quantity $\phi_{d}$, which equals $2 \beta$ in the $\mathrm{SM}$, is the CP-violating weak $B_{d}^{0}-\bar{B}_{d}^{0}$ mixing phase. Consequently, we arrive at the following expressions:

$$
\begin{gathered}
\mathcal{A}_{\mathrm{CP}}^{\mathrm{dir}}\left(B_{d} \rightarrow \pi^{+} \pi^{-}\right)=-\left[\frac{2 d \sin \theta \sin \gamma}{1-2 d \cos \theta \cos \gamma+d^{2}}\right] \\
\mathcal{A}_{\mathrm{CP}}^{\mathrm{mix}}\left(B_{d} \rightarrow \pi^{+} \pi^{-}\right)=\frac{\sin \left(\phi_{d}+2 \gamma\right)-2 d \cos \theta \sin \left(\phi_{d}+\gamma\right)+d^{2} \sin \phi_{d}}{1-2 d \cos \theta \cos \gamma+d^{2}} .
\end{gathered}
$$


The current experimental status of these observables is given by

$$
\begin{aligned}
& \mathcal{A}_{\mathrm{CP}}^{\mathrm{dir}}\left(B_{d} \rightarrow \pi^{+} \pi^{-}\right)=\left\{\begin{array}{l}
-0.19 \pm 0.19 \pm 0.05 \text { (BaBar [65]) } \\
-0.77 \pm 0.27 \pm 0.08 \text { (Belle [66]) }
\end{array}\right. \\
& \mathcal{A}_{\mathrm{CP}}^{\text {mix }}\left(B_{d} \rightarrow \pi^{+} \pi^{-}\right)=\left\{\begin{array}{l}
+0.40 \pm 0.22 \pm 0.03 \text { (BaBar [65]) } \\
+1.23 \pm 0.41_{-0.08}^{+0.07} \text { (Belle [66]). }
\end{array}\right.
\end{aligned}
$$

Unfortunately, the BaBar and Belle results are not fully consistent with each other, although both experiments point towards the same signs, and the last BaBar update of $\mathcal{A}_{\mathrm{CP}}^{\mathrm{mix}}\left(B_{d} \rightarrow \pi^{+} \pi^{-}\right)$has moved towards Belle. In [11], the following averages are given:

$$
\begin{aligned}
& \mathcal{A}_{\mathrm{CP}}^{\mathrm{dir}}\left(B_{d} \rightarrow \pi^{+} \pi^{-}\right)=-0.38 \pm 0.16 \\
& \mathcal{A}_{\mathrm{CP}}^{\mathrm{mix}}\left(B_{d} \rightarrow \pi^{+} \pi^{-}\right)=+0.58 \pm 0.20 .
\end{aligned}
$$

As was pointed out in $[55,56]$, the $\mathrm{CP}$ asymmetries in (3.29) and (3.30) point towards $\gamma \sim 60^{\circ}$ for $\phi_{d} \sim 47^{\circ}$, in accordance with the SM. Before discussing the determination of $\gamma$ in more detail in Subsection 3.4, ${ }^{4}$ it is useful to first have a closer look at the hadronic $B \rightarrow \pi \pi$ parameters.

\subsection{Determination of the Hadronic Parameters}

In order to explore the hadronic $B \rightarrow \pi \pi$ parameters, we assume that

$$
\gamma=(65 \pm 7)^{\circ}, \quad \phi_{d}=2 \beta=(47 \pm 4)^{\circ}
$$

as in the SM [4]. If we then look at (3.25) and (3.26), we observe that each of these observables allows us to determine $d$ as a function of the strong phase $\theta$. In the case of the direct $\mathrm{CP}$ asymmetry $\mathcal{A}_{\mathrm{CP}}^{\mathrm{dir}}\left(B_{d} \rightarrow \pi^{+} \pi^{-}\right)$, we obtain

$$
\begin{aligned}
d & =\frac{1}{\mathcal{A}_{\mathrm{CP}}^{\mathrm{dir}}\left(B_{d} \rightarrow \pi^{+} \pi^{-}\right)}\left[\mathcal{A}_{\mathrm{CP}}^{\mathrm{dir}}\left(B_{d} \rightarrow \pi^{+} \pi^{-}\right) \cos \theta \cos \gamma-\sin \theta \sin \gamma\right. \\
& \left. \pm \sqrt{\left[\mathcal{A}_{\mathrm{CP}}^{\mathrm{dir}}\left(B_{d} \rightarrow \pi^{+} \pi^{-}\right) \cos \theta \cos \gamma-\sin \theta \sin \gamma\right]^{2}-\mathcal{A}_{\mathrm{CP}}^{\operatorname{dir}}\left(B_{d} \rightarrow \pi^{+} \pi^{-}\right)^{2}}\right]
\end{aligned}
$$

whereas its mixing-induced counterpart $\mathcal{A}_{\mathrm{CP}}^{\mathrm{mix}}\left(B_{d} \rightarrow \pi^{+} \pi^{-}\right)$implies

$$
d=k \pm \sqrt{k^{2}-l}
$$

with

$$
\begin{gathered}
k=\left[\frac{\sin \left(\phi_{d}+\gamma\right)-\mathcal{A}_{\mathrm{CP}}^{\mathrm{mix}}\left(B_{d} \rightarrow \pi^{+} \pi^{-}\right) \cos \gamma}{\sin \phi_{d}-\mathcal{A}_{\mathrm{CP}}^{\mathrm{mix}}\left(B_{d} \rightarrow \pi^{+} \pi^{-}\right)}\right] \cos \theta \\
l=\frac{\sin \left(\phi_{d}+2 \gamma\right)-\mathcal{A}_{\mathrm{CP}}^{\mathrm{mix}}\left(B_{d} \rightarrow \pi^{+} \pi^{-}\right)}{\sin \phi_{d}-\mathcal{A}_{\mathrm{CP}}^{\mathrm{mix}}\left(B_{d} \rightarrow \pi^{+} \pi^{-}\right)} .
\end{gathered}
$$

In Fig. 1, we show the corresponding contours, and observe that we obtain a twofold solution for $(d, \theta)$. It should be emphasized that these contours and the corresponding determination of $(d, \theta)$ for a given value of $\gamma$ are theoretically clean.

\footnotetext{
${ }^{4}$ See also Appendix B, where a very recent update by the Belle collaboration [67] is discussed.
} 


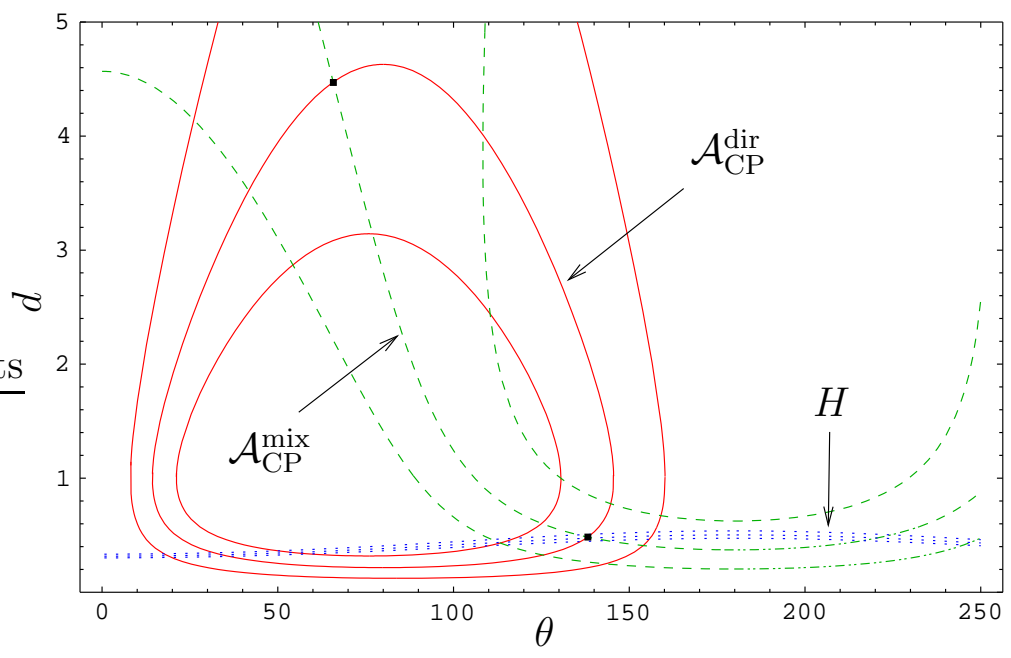

Figure 1: The contours in the $\theta-d$ plane for $\gamma=65^{\circ}$ and $\phi_{d}=47^{\circ}$. The solid lines correspond to the central value and $1 \sigma$ upper and lower ranges of $\mathcal{A}_{\mathrm{CP}}^{\mathrm{dir}}\left(B_{d} \rightarrow \pi^{+} \pi^{-}\right)=$ $-0.38 \pm 0.16$, the dashed lines represent $\mathcal{A}_{\mathrm{CP}}^{\operatorname{mix}}\left(B_{d} \rightarrow \pi^{+} \pi^{-}\right)=+0.58 \pm 0.20$, and the dotted lines refer to $H=7.17 \pm 0.75$, as discussed in Subsection 3.4.

Let us now also consider the observables $R_{+-}^{\pi \pi}$ and $R_{00}^{\pi \pi}$. If we complement them with the CP-violating observables $\mathcal{A}_{\mathrm{CP}}^{\text {dir }}\left(B_{d} \rightarrow \pi^{+} \pi^{-}\right)$and $\mathcal{A}_{\mathrm{CP}}^{\text {mix }}\left(B_{d} \rightarrow \pi^{+} \pi^{-}\right)$, which allow us to determine $d$ and $\theta$ as we have just seen, we may extract the parameters $x$ and $\Delta$ as well. To this end, it is convenient to introduce

$$
\begin{gathered}
\tilde{R}_{+-}^{\pi \pi} \equiv D R_{+-}^{\pi \pi} \\
\tilde{R}_{00}^{\pi \pi} \equiv D R_{00}^{\pi \pi},
\end{gathered}
$$

with

$$
D \equiv 1-2 d \cos \theta \cos \gamma+d^{2} .
$$

Using then (3.20) and (3.21), we obtain

$$
x=-\cos \Delta \pm \sqrt{\tilde{R}_{+-}^{\pi \pi}-\sin ^{2} \Delta}
$$

and

$$
x=-d \cos \gamma \cos (\Delta-\theta) \pm \sqrt{\tilde{R}_{00}^{\pi \pi}-\left[1-\cos ^{2} \gamma \cos ^{2}(\Delta-\theta)\right] d^{2}},
$$

respectively, allowing us to calculate two contours in the $\Delta-x$ plane. The intersections of these curves then allow us to extract $x$ and $\Delta$. Interestingly, the $(d, \theta)$ solution with $d \sim 5$ gives only complex solutions and can therefore by excluded. On the other hand, in Fig. 2, we show the contours in the $\Delta-x$ plane corresponding to the physical $d \sim 0.5$ case, yielding a twofold solution for $(x, \Delta)$.

Using (3.31) as an input, the expressions in (3.20), (3.21), (3.25) and (3.26) allow us to convert the data in $(1.1),(1.2),(3.29)$ and (3.30) into the hadronic parameters $(d, \theta)$ and $(x, \Delta)$. Following these lines, we obtain

$$
d=0.48_{-0.22}^{+0.35}, \quad \theta=+\left(138_{-23}^{+19}\right)^{\circ},
$$




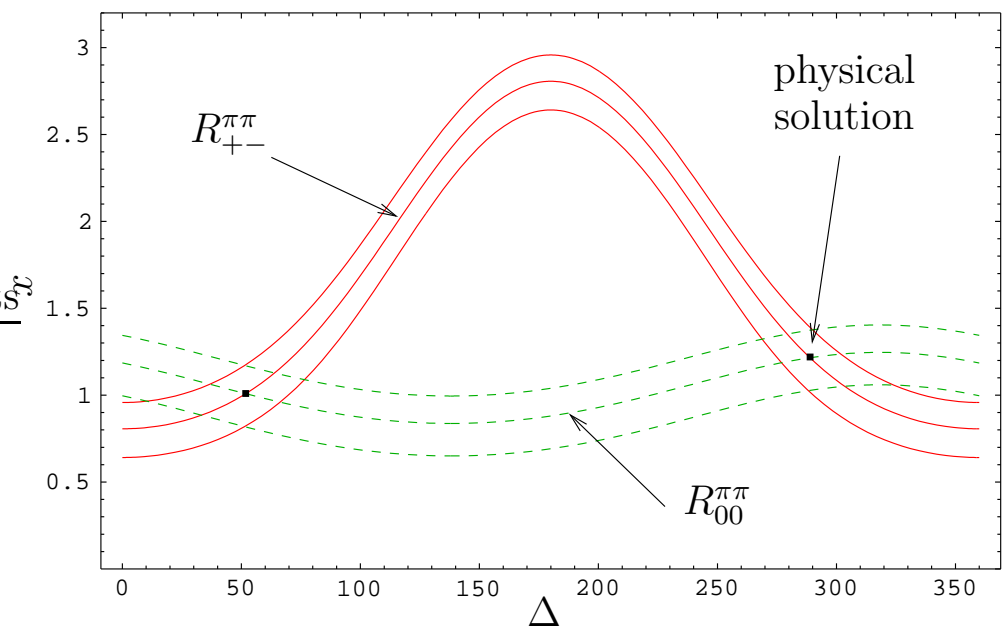

Figure 2: The contours in the $\Delta-x$ plane. Using $d=0.48$ and $\theta=138^{\circ}$ following from the central values of Fig. 1, we obtain the solid set of contours for $R_{+-}^{\pi \pi}=2.12 \pm 0.37$ and the dashed set of contours for $R_{00}^{\pi \pi}=0.83 \pm 0.23$.

as well as the twofold solution

$$
\begin{array}{ll}
x=1.22_{-0.21}^{+0.26}, & \Delta=-\left(71_{-26}^{+19}\right)^{\circ}, \\
x=1.01_{-0.19}^{+0.25}, & \Delta=+\left(52_{-34}^{+24}\right)^{\circ},
\end{array}
$$

where our treatment of errors is as described in Appendix C. At this stage, we cannot distinguish between the two solutions for $x e^{i \Delta}$. However, as we will show in Subsection 4.4.3, the solution in (3.43) can be excluded through the $B \rightarrow \pi K$ data, as it would correspond to large direct CP violation in $B^{ \pm} \rightarrow \pi^{0} K^{ \pm}$, which is ruled out by experiment. For the time being, we will hence focus on (3.42). Let us finally note that the determination of the hadronic parameters given in (3.41)-(3.43) is essentially theoretically clean, and that the experimental picture will improve significantly in the future.

\subsection{Interpretation of the Hadronic Parameters}

The result of $x=\mathcal{O}(1)$ obtained above, which implies that $|\tilde{C}| \sim|\tilde{T}|$, is in stark contrast to the naïve expectation of $|\tilde{C}| \sim 0.25 \times|\tilde{T}|$. At first sight, this feature seems to imply a complete breakdown of the concept of colour suppression in $B \rightarrow \pi \pi$ decays. However, one should realize that the usual arguments leading to $|\tilde{C}| \sim 0.25 \times|\tilde{T}|$ neglect the contributions $\left(\mathcal{P}_{t u}-\mathcal{E}\right)$ in (3.5) and (3.6), which may be important [64]. In fact, our analysis suggests that these contributions cannot be neglected if we want to understand the pattern of the $B \rightarrow \pi \pi$ observables in a plausible manner. Because of the different signs in (3.5) and (3.6), we may explain the surprisingly small $B_{d} \rightarrow \pi^{+} \pi^{-}$branching ratio naturally through destructive interference between the $\mathcal{T}$ and $\left(\mathcal{P}_{t u}-\mathcal{E}\right)$ amplitudes, whereas the puzzling large $B_{d} \rightarrow \pi^{0} \pi^{0}$ branching ratio originates from constructive interference between the $\mathcal{C}$ and $\left(\mathcal{P}_{t u}-\mathcal{E}\right)$ amplitudes. 
On the other hand, we have $\left.\theta\right|_{\text {fact }}=180^{\circ}$. Consequently, as can be seen in (3.12), $B_{d} \rightarrow \pi^{+} \pi^{-}$would favour - in contrast to the SM expectation $-\gamma>90^{\circ}$ within the factorization approach, since $\mathrm{BR}\left(B_{d} \rightarrow \pi^{+} \pi^{-}\right)$would then be reduced through destructive interference between trees and penguins. In contrast, we arrive at a picture with large non-factorizable contributions, exhibiting certain interference effects at the hadronic level that allow us to accommodate straightforwardly any currently observed feature of the $B \rightarrow \pi \pi$ system in the SM.

\subsubsection{Insights into the Substructure of $x e^{i \Delta}$}

The calculation of the hadronic parameter $x e^{i \Delta}$ from first principles is extremely challenging and cannot be done in a reliable manner because of our limited knowledge of non-perturbative strong-interaction physics. However, in order to obtain deeper insights into the substructure of $x e^{i \Delta}$, it is instructive to write

$$
x e^{i \Delta}=\frac{a_{2}^{\pi \pi} e^{i \Delta_{2}^{\pi \pi}}+\zeta e^{i \Delta_{\zeta}}}{1-\zeta e^{i \Delta_{\zeta}}},
$$

with

$$
\begin{gathered}
a_{2}^{\pi \pi} e^{i \Delta_{2}^{\pi \pi}} \equiv \frac{\mathcal{C}}{\mathcal{T}} \\
\zeta e^{i \Delta_{\zeta}} \equiv \frac{\mathcal{P}_{t u}-\mathcal{E}}{\mathcal{T}}
\end{gathered}
$$

Consequently, we may convert $x e^{i \Delta}$ into the complex quantity $\zeta e^{i \Delta_{\zeta}}$ with the help of

$$
\zeta e^{i \Delta_{\zeta}}=\frac{x^{2}+x\left[e^{i \Delta}-a_{2}^{\pi \pi} e^{i\left(\Delta_{2}^{\pi \pi}-\Delta\right)}\right]-a_{2}^{\pi \pi} e^{i \Delta_{2}^{\pi \pi}}}{1+2 x \cos \Delta+x^{2}} .
$$

In the special case of $a_{2}^{\pi \pi} e^{i \Delta_{2}^{\pi \pi}}=x e^{i \Delta}$, this expression reduces to $\zeta e^{i \Delta_{\zeta}}=0$. However, a value of $a_{2}^{\pi \pi} e^{i \Delta_{2}^{\pi \pi}} \sim 1.22 \times e^{-i 71^{\circ}}$ would appear as completely unrealistic. On the other hand, in view of the large non-factorizable effects exhibited by (3.41) and (3.42), we think that $a_{2}^{\pi \pi}$ may well take values as large as 0.5 , with a large strong phase $\Delta_{2}^{\pi \pi}$. In Fig. 3 , we consider the central values of $x$ and $\Delta$ in (3.42), and show the contours in the $\Delta_{\zeta} \zeta$ plane corresponding to different values of $a_{2}^{\pi \pi}$, where each point is parametrized by the value of $\Delta_{2}^{\pi \pi} \in\left[0^{\circ}, 360^{\circ}\right]$. We observe that the "naïve" value of $a_{2}^{\pi \pi} e^{i \Delta_{2}^{\pi \pi}} \sim 0.25$ would result in a rather large value of $\zeta \sim 0.65$. On the other hand, $a_{2}^{\pi \pi} e^{i \Delta_{2}^{\pi \pi}} \sim 0.5 \times e^{i 290^{\circ}}$ gives a significantly smaller $\zeta \sim 0.4$, which may well originate from constructive interference between the $\mathcal{P}_{t}$ and $\mathcal{P}_{u}$ amplitudes, satisfying, for instance, $\left|\mathcal{P}_{t} / \mathcal{T}\right| \sim\left|\mathcal{P}_{u} / \mathcal{T}\right| \sim 0.25$. Moreover, for such values of $a_{2}^{\pi \pi} e^{i \Delta_{2}^{\pi \pi}}$, we have $\left|1+a_{2}^{\pi \pi} e^{i \Delta_{2}^{\pi \pi}}\right| \sim 1.25$, in accordance with the "naïve" expectation. Consequently, in the $B^{ \pm} \rightarrow \pi^{ \pm} \pi^{0}$ channel, which is measured in agreement with the conventional theoretical expectations, not only the hadronic interference effects originating from the $\left(\mathcal{P}_{t u}-\mathcal{E}\right)$ amplitude cancel, but also the large non-factorizable effects in $a_{2}^{\pi \pi} e^{i \Delta_{2}^{\pi \pi}}$ would not manifest themselves.

We shall come back to these considerations in Subsection 4.2.3, where the quantity

$$
\tilde{\zeta} e^{i \Delta_{\tilde{\zeta}}} \equiv \frac{\zeta e^{i \Delta_{\zeta}}}{1-\zeta e^{i \Delta_{\zeta}}}=\frac{\mathcal{P}_{t u}-\mathcal{E}}{\tilde{\mathcal{T}}}
$$




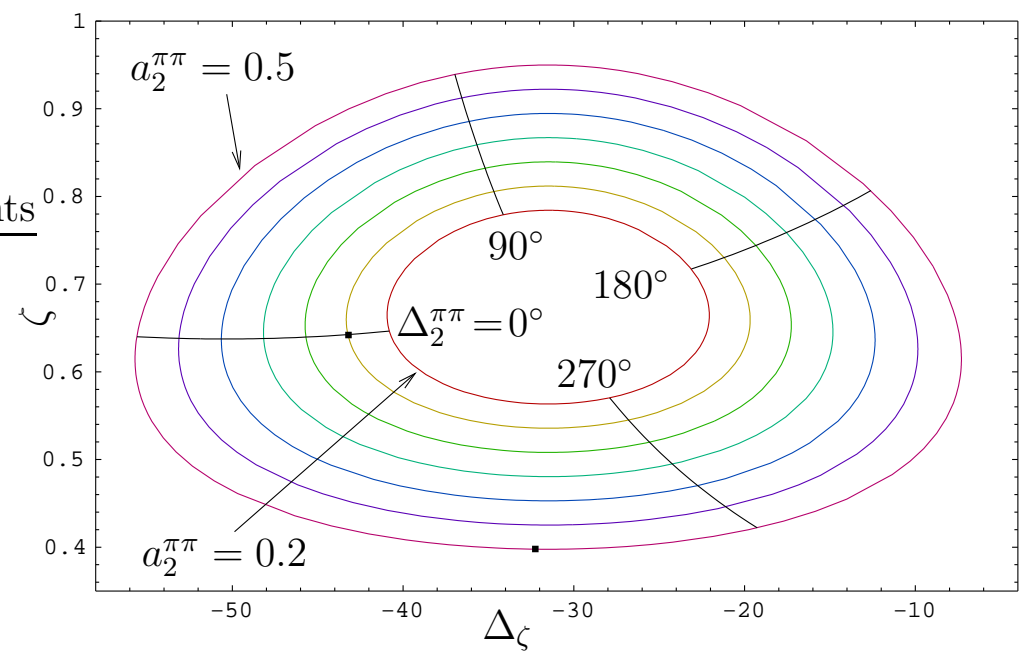

Figure 3: The contours in the $\Delta_{\zeta} \zeta$ plane corresponding to the central values of $x e^{i \Delta}$ in (3.42) for various values of $a_{2}^{\pi \pi}$ between 0.2 and 0.5 and $\Delta_{2}^{\pi \pi} \in\left[0^{\circ}, 360^{\circ}\right]$. The two dots refer to cases discussed in the text.

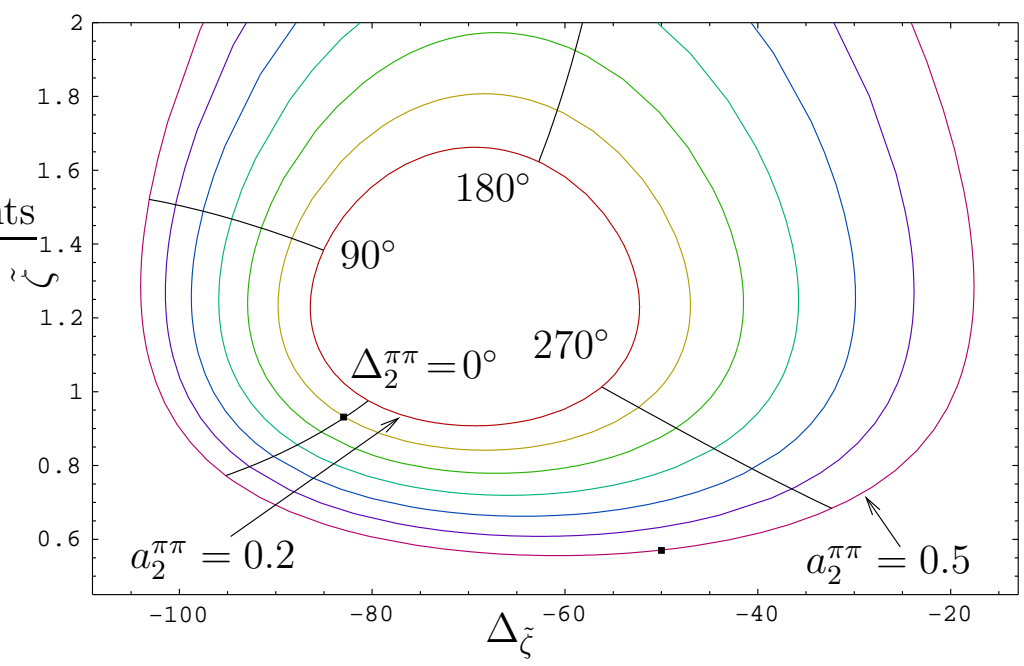

Figure 4: The contours in the $\Delta_{\tilde{\zeta}} \tilde{\zeta}$ plane corresponding to the central values of $x e^{i \Delta}$ in (3.42) for various values of $a_{2}^{\pi \pi}$ between 0.2 and 0.5 and $\Delta_{2}^{\pi \pi} \in\left[0^{\circ}, 360^{\circ}\right]$. 
will play an important rôle in the context of a hadronic parameter $\rho_{\mathrm{c}}$ entering the charged $B \rightarrow \pi K$ decays. In Fig. 4 , we show - in analogy to Fig. 3 - the contour plots in the $\Delta_{\tilde{\zeta}}-\tilde{\zeta}$ plane for given values of $a_{2}^{\pi \pi}$ and $\Delta_{2}^{\pi \pi} \in\left[0^{\circ}, 360^{\circ}\right]$. Interestingly, the analysis of $\rho_{\mathrm{c}}$ will also point towards $a_{2}^{\pi \pi} e^{i \Delta_{2}^{\pi \pi}} \sim 0.5 \times e^{i 290^{\circ}}$, thereby complementing the picture described above.

Let us finally note that the hadronic parameters in (3.41) and (3.42) also imply

$$
\left[\frac{P}{T+C}\right] e^{i \gamma}=-\left[\frac{d e^{i \theta}}{1+x e^{i \Delta}}\right]=\frac{1}{R_{b}}\left[\frac{\mathcal{P}_{t c}}{\mathcal{T}+\mathcal{C}}\right]=\left(0.27_{-0.10}^{+0.13}\right) \times e^{i\left(-2_{-23}^{+18}\right)^{\circ}},
$$

yielding

$$
\frac{\mathcal{P}_{t}-\mathcal{P}_{c}}{\mathcal{T}+\mathcal{C}}=\left(0.10_{-0.04}^{+0.05}\right) \times e^{i\left(-2_{-23}^{+18}\right)^{\circ}}
$$

where we have used the value of $R_{b}$ in (2.19). Consequently, the small numerical value in (3.50) would then require destructive interference between the $\mathcal{P}_{t}$ and $\mathcal{P}_{c}$ amplitudes, where $\mathcal{P}_{c}$ contains "charming" penguins [68], in contrast to the constructive interference between $\mathcal{P}_{t}$ and $\mathcal{P}_{u}$. Interesting insights into these subtle dynamical issues can be obtained with the help of the penguin-induced decay $B_{d} \rightarrow K^{0} \bar{K}^{0}$ [69], which can nicely be complemented with the $B_{s} \rightarrow K^{0} \bar{K}^{0}$ channel [2].

\subsubsection{Probing Penguin Annihilation and Exchange Topologies through the $\boldsymbol{B}_{\boldsymbol{d}} \rightarrow \boldsymbol{K}^{+} \boldsymbol{K}^{-}, \boldsymbol{B}_{s} \rightarrow \boldsymbol{\pi}^{+} \boldsymbol{\pi}^{-}$System}

The origin of the value of $x e^{i \Delta}$ in (3.42) could also be due to anomalously enhanced penguin annihilation and exchange topologies (which contribute to the $B \rightarrow \pi \pi$ modes but not to their $B \rightarrow \pi K$ counterparts discussed in Section 4). However, the importance of these topologies can be probed through the decay $B_{d} \rightarrow K^{+} K^{-}$, where the current experimental upper bound is given as follows [11]:

$$
\operatorname{BR}\left(B_{d} \rightarrow K^{+} K^{-}\right)<0.6 \times 10^{-6}(90 \% \text { C.L. }) .
$$

The corresponding decay amplitude has the same structure as (3.12), with the important difference that now only penguin annihilation and exchange topologies contribute. Employing the $S U(3)$ flavour symmetry of strong interactions, we may hence write

$$
A\left(B_{d}^{0} \rightarrow K^{+} K^{-}\right)=-\lambda^{3} A R_{b}\left[\mathcal{E}-(\mathcal{P} \mathcal{A})_{t u}\right]\left[e^{i \gamma}+\varrho_{\mathcal{P} \mathcal{A}} e^{i \vartheta_{\mathcal{P} \mathcal{A}}}\right]
$$

with

$$
\varrho_{\mathcal{P} \mathcal{A}} e^{i \vartheta_{\mathcal{P} \mathcal{A}}} \equiv \frac{1}{R_{b}}\left[\frac{(\mathcal{P} \mathcal{A})_{t c}}{\mathcal{E}-(\mathcal{P} \mathcal{A})_{t u}}\right]
$$

where the penguin annihilation amplitudes $(\mathcal{P} \mathcal{A})_{t u}$ and $(\mathcal{P} \mathcal{A})_{t c}$ are implicitly included in the $B \rightarrow \pi \pi$ amplitudes $\mathcal{P}_{t u}$ and $\mathcal{P}_{t c}$, respectively. Consequently, we obtain

$$
\sqrt{\frac{1}{2}\left[\frac{\mathrm{BR}\left(B_{d} \rightarrow K^{+} K^{-}\right)}{\operatorname{BR}\left(B^{ \pm} \rightarrow \pi^{ \pm} \pi^{0}\right)}\right] \frac{\tau_{B^{+}}}{\tau_{B_{d}^{0}}}} \approx\left|\frac{\mathcal{E}-(\mathcal{P} \mathcal{A})_{t u}}{\mathcal{T}+\mathcal{C}}\right| \sqrt{1+2 \varrho_{\mathcal{P} \mathcal{A}} \cos \vartheta_{\mathcal{P} \mathcal{A}} \cos \gamma+\varrho_{\mathcal{P} \mathcal{A}}^{2}} \lesssim 0.2,
$$


which does not indicate any anomalous behaviour. In the future, this bound can be improved significantly. The decay $B_{d} \rightarrow K^{+} K^{-}$is very accessible at LHCb, where it may be possible to reach the $10^{-8}$ level for its $\mathrm{CP}$-averaged branching ratio [70]. At this experiment, we may also exploit the physics potential of the $B_{s}^{0} \rightarrow \pi^{+} \pi^{-}$channel, which is the $U$-spin partner of the $B_{d}^{0} \rightarrow K^{+} K^{-}$mode, ${ }^{5}$ and has a decay amplitude of the same structure as the $B_{s}^{0} \rightarrow K^{+} K^{-}$mode [53] (or the $B_{d}^{0} \rightarrow \pi^{-} K^{+}$transition discussed in Section 4). If we then use the $U$-spin flavour symmetry and consider the CP-averaged branching ratios, we obtain

$$
H_{\mathcal{P} \mathcal{A}} \equiv \frac{1}{\epsilon}\left[\frac{\mathrm{BR}\left(B_{d} \rightarrow K^{+} K^{-}\right)}{\mathrm{BR}\left(B_{s} \rightarrow \pi^{+} \pi^{-}\right)}\right] \frac{\tau_{B_{s}^{0}}}{\tau_{B_{d}^{0}}}=\frac{1+2 \varrho_{\mathcal{P} \mathcal{A}} \cos \vartheta_{\mathcal{P} \mathcal{A}} \cos \gamma+\varrho_{\mathcal{P} \mathcal{A}}^{2}}{\epsilon^{2}-2 \epsilon \varrho_{\mathcal{P} \mathcal{A}} \cos \vartheta_{\mathcal{P} \mathcal{A}} \cos \gamma+\varrho_{\mathcal{P} \mathcal{A}}^{2}}
$$

where we neglect tiny phase-space effects, and

$$
\epsilon \equiv \frac{\lambda^{2}}{1-\lambda^{2}}=0.053
$$

The quantity $H_{\mathcal{P} \mathcal{A}}$ allows us to obtain constraints for the parameter $\varrho_{\mathcal{P} \mathcal{A}}$. Since the amplitude structure of the $B_{d} \rightarrow K^{+} K^{-}, B_{s} \rightarrow \pi^{+} \pi^{-}$system is analogous to that of the $B_{d} \rightarrow \pi^{+} \pi^{-}, B_{s} \rightarrow K^{+} K^{-}\left(B_{d} \rightarrow \pi^{\mp} K^{ \pm}\right)$system, we may apply the bounds derived in [54], yielding

$$
\frac{1-\epsilon \sqrt{H_{\mathcal{P} \mathcal{A}}}}{1+\sqrt{H_{\mathcal{P A}}}} \leq \varrho_{\mathcal{P} \mathcal{A}} \leq \frac{1+\epsilon \sqrt{H_{\mathcal{P} \mathcal{A}}}}{\left|1-\sqrt{H_{\mathcal{P} \mathcal{A}}}\right|}
$$

In complete analogy to the $B_{d} \rightarrow \pi^{+} \pi^{-}, B_{s} \rightarrow K^{+} K^{-}$strategy proposed in [53], a measurement of the CP-violating $B_{d} \rightarrow K^{+} K^{-}, B_{s} \rightarrow \pi^{+} \pi^{-}$observables would allow a determination of both $\gamma$ and the hadronic parameters $\varrho_{\mathcal{P} \mathcal{A}}$ and $\vartheta_{\mathcal{P} \mathcal{A}}$. However, the main interest in an analysis of the $B_{d} \rightarrow K^{+} K^{-}, B_{s} \rightarrow \pi^{+} \pi^{-}$system is obviously to obtain insights into the importance of penguin annihilation and exchange topologies. Using the corresponding information on $\varrho_{\mathcal{P} \mathcal{A}}$ and $\vartheta_{\mathcal{P} \mathcal{A}}$, the expression in (3.54) allows us to determine $\left|\left(\mathcal{E}-(\mathcal{P} \mathcal{A})_{t u}\right) /(\mathcal{T}+\mathcal{C})\right|$, while

$$
\sqrt{\frac{\epsilon}{2}\left[\frac{\operatorname{BR}\left(B_{s} \rightarrow \pi^{+} \pi^{-}\right)}{\operatorname{BR}\left(B^{ \pm} \rightarrow \pi^{ \pm} \pi^{0}\right)}\right] \frac{\tau_{B^{+}}}{\tau_{B_{s}^{0}}}} \approx \frac{1}{R_{b}}\left|\frac{(\mathcal{P} \mathcal{A})_{t c}}{\mathcal{T}+\mathcal{C}}\right| .
$$

It will be very interesting to confront these considerations with experimental data for the $B_{s} \rightarrow \pi^{+} \pi^{-}$channel. First constraints may soon be available from run II of the Tevatron, which should be considerably improved at LHCb.

\subsection{Determination of $\gamma$}

Let us now come back to Fig. 1. There we have also included a contour, which is related to the following quantity [54]:

$$
H \equiv \frac{1}{\epsilon}\left|\frac{\mathcal{C}^{\prime}}{\mathcal{C}}\right|^{2}\left[\frac{M_{B_{d}}}{M_{B_{s}}} \frac{\Phi\left(M_{K} / M_{B_{s}}, M_{K} / M_{B_{s}}\right)}{\Phi\left(M_{\pi} / M_{B_{d}}, M_{\pi} / M_{B_{d}}\right)} \frac{\tau_{B_{s}^{0}}}{\tau_{B_{d}^{0}}}\right]\left[\frac{\mathrm{BR}\left(B_{d} \rightarrow \pi^{+} \pi^{-}\right)}{\operatorname{BR}\left(B_{s} \rightarrow K^{+} K^{-}\right)}\right]
$$

\footnotetext{
${ }^{5}$ The $U$-spin flavour symmetry of strong interactions connects the down and strange quarks in the same manner as the ordinary $S U(2)$ isospin flavour symmetry connects the down and up quarks.
} 


$$
\stackrel{U \text { spin }}{=} \frac{1-2 d \cos \theta \cos \gamma+d^{2}}{\epsilon^{2}+2 \epsilon d \cos \theta \cos \gamma+d^{2}},
$$

where $\left|\mathcal{C}^{\prime} / \mathcal{C}\right|$ is a $U$-spin-breaking parameter, and

$$
\Phi(x, y) \equiv \sqrt{\left[1-(x+y)^{2}\right]\left[1-(x-y)^{2}\right]}
$$

denotes the usual two-body phase-space function. The introduction and the use of $H$, which has the same structure as (3.55), is inspired by a variant of the strategy presented in [53], allowing us to determine $\gamma$ and the hadronic parameters $(d, \theta)$ by relating the CP-violating $B_{d} \rightarrow \pi^{+} \pi^{-}$observables to their $B_{s} \rightarrow K^{+} K^{-}$counterparts through the $U$-spin flavour symmetry of strong interactions (see also Subsection 4.6.1). This strategy is very promising for $B$-decay experiments at hadron colliders. First important steps are already expected at run II of the Tevatron [71], whereas it can be fully exploited in the era of the LHC [72], in particular at LHCb, where experimental uncertainties of $\gamma$ at the few-degree level may be achieved [73]. Unfortunately, the physics potential of $B_{s} \rightarrow K^{+} K^{-}$cannot yet be exploited. However, if we assume that the penguin annihilation and exchange topologies discussed in Subsection 3.3 .2 play a minor rôle and employ $S U(3)$ flavour-symmetry arguments, we may replace $B_{s} \rightarrow K^{+} K^{-}$through $B_{d} \rightarrow \pi^{\mp} K^{ \pm}$, and arrive straightforwardly at

$$
H=\frac{1}{\epsilon}\left(\frac{f_{K}}{f_{\pi}}\right)^{2}\left[\frac{\mathrm{BR}\left(B_{d} \rightarrow \pi^{+} \pi^{-}\right)}{\operatorname{BR}\left(B_{d} \rightarrow \pi^{\mp} K^{ \pm}\right)}\right]=7.17 \pm 0.75,
$$

where the ratio $f_{K} / f_{\pi}=160 / 131$ describes factorizable $S U(3)$-breaking corrections [54]. In fact, the utility of $B_{d} \rightarrow \pi^{\mp} K^{ \pm}$transitions to address the "penguin problem" in $B_{d} \rightarrow \pi^{+} \pi^{-}$was already pointed out about ten years ago [74]. If we now use the expression for $H$ in terms of the hadronic parameters given in (3.59), we obtain [54]:

$$
d=-b \cos \theta \cos \gamma \pm \sqrt{a+(b \cos \theta \cos \gamma)^{2}}
$$

with

$$
a=\frac{1-\epsilon^{2} H}{H-1}, \quad b=\frac{1+\epsilon H}{H-1},
$$

which allows us to calculate $d$ as a function of $\theta$ for $\gamma$ and $H$ as given in (3.31) and (3.61), respectively. The resulting contour is shown in Fig. 1 and fits perfectly into the picture of the theoretically clean $\theta-d$ contours following from the CP-violating $B_{d} \rightarrow$ $\pi^{+} \pi^{-}$observables. The fact that the $H$ contour described by (3.62) goes right through the intersection of (3.32) and (3.33) is very remarkable and indicates that the penguin annihilation and exchange amplitudes as well as non-factorizable $S U(3)$-breaking effects actually play a minor rôle. Otherwise, the corresponding hadronic uncertainties would have to conspire in a very contrived way, which does not look plausible to us.

If we do not fix $\gamma$ through the UT fits, but use instead $H$, as discussed in [53]-[56], we may determine this angle, yielding the following twofold solution:

$$
\gamma=\left(40.5_{-6.1}^{+5.4}\right)^{\circ} \vee\left(64.7_{-6.9}^{+6.3}\right)^{\circ}
$$


The errors are obtained with the help of a standard $\chi^{2}$ analysis from the errors of $\mathcal{A}_{\mathrm{CP}}^{\text {dir }}\left(B_{d} \rightarrow \pi^{+} \pi^{-}\right), \mathcal{A}_{\mathrm{CP}}^{\mathrm{mix}}\left(B_{d} \rightarrow \pi^{+} \pi^{-}\right)$and $H$ by fitting to the hadronic parameters and $\gamma$. In addition, there is an error of $\pm 2.5^{\circ}$ on $\gamma$ from the uncertainty in $\phi_{d}$. A detailed analysis of the whole $B \rightarrow \pi K$ system, as discussed in Subsection 4.3, allows us to lift this degeneracy, leaving us essentially with the solution around $65^{\circ}$, which agrees perfectly with the range for $\gamma$ given in (3.31).

An interesting alternative to determine $\gamma$ is provided by the direct $\mathrm{CP}$ asymmetry

$$
\mathcal{A}_{\mathrm{CP}}^{\mathrm{dir}}\left(B_{d} \rightarrow \pi^{\mp} K^{ \pm}\right) \equiv \frac{\mathrm{BR}\left(B_{d}^{0} \rightarrow \pi^{-} K^{+}\right)-\mathrm{BR}\left(\bar{B}_{d}^{0} \rightarrow \pi^{+} K^{-}\right)}{\operatorname{BR}\left(B_{d}^{0} \rightarrow \pi^{-} K^{+}\right)+\operatorname{BR}\left(\bar{B}_{d}^{0} \rightarrow \pi^{+} K^{-}\right)}=+0.095 \pm 0.028 .
$$

If we employ again the $S U(3)$ flavour symmetry and the plausible dynamical arguments specified above, we may express this observable in terms of the hadronic parameters introduced in (3.15) as follows:

$$
\mathcal{A}_{\mathrm{CP}}^{\mathrm{dir}}\left(B_{d} \rightarrow \pi^{\mp} K^{ \pm}\right)=\frac{2 \epsilon d \sin \theta \sin \gamma}{\epsilon^{2}+2 \epsilon d \cos \theta \cos \gamma+d^{2}} .
$$

In contrast to expression (3.61) for $H,(3.66)$ does not involve a $\left(f_{K} / f_{\pi}\right)^{2}$ term and is hence affected to a smaller extent by $S U(3)$-breaking corrections, i.e. is more favourable from a theoretical point of view. On the other hand, as can be seen in (3.65), a nonvanishing value of $\mathcal{A}_{\mathrm{CP}}^{\mathrm{dir}}\left(B_{d} \rightarrow \pi^{\mp} K^{ \pm}\right)$has not yet been established by the $B$ factories, although the experimental evidence for this $\mathrm{CP}$ asymmetry is steadily increasing.

In this context, it is important to note that we have the following relation $[53,54]$ :

$$
\underbrace{-\left[\frac{\mathcal{A}_{\mathrm{CP}}^{\mathrm{dir}}\left(B_{d} \rightarrow \pi^{\mp} K^{ \pm}\right)}{\mathcal{A}_{\mathrm{CP}}^{\mathrm{dir}}\left(B_{d} \rightarrow \pi^{+} \pi^{-}\right)}\right]}_{0.25 \pm 0.13}=\underbrace{\left(\frac{f_{K}}{f_{\pi}}\right)^{2}\left[\frac{\mathrm{BR}\left(B_{d} \rightarrow \pi^{+} \pi^{-}\right)}{\mathrm{BR}\left(B_{d} \rightarrow \pi^{\mp} K^{ \pm}\right)}\right]}_{0.38 \pm 0.04}=\epsilon H,
$$

where we have also indicated the current experimental results. Within the uncertainties, this relation is satisfied by the data. In particular, also the pattern of the signs of the direct $\mathrm{CP}$ asymmetries is in accordance with (3.67). Should the central values be correct, they would correspond to non-factorizable $S U(3)$-breaking corrections at the $B \rightarrow \pi \pi, \pi K$ amplitude level of $\mathcal{O}(20 \%)$. On the other hand, the current central value for $\mathcal{A}_{\mathrm{CP}}^{\mathrm{dir}}\left(B_{d} \rightarrow \pi^{\mp} K^{ \pm}\right)$may also be on the lower side, which would be our preferred scenario. It will be exciting to observe how the data evolve in the future.

In our analysis, we have assumed that $\phi_{d} \sim 47^{\circ}$, in agreement with the SM. However, as discussed in $[55,56]$, it is interesting to consider also the second, unconventional solution of $\phi_{d} \sim 133^{\circ}$. If $\phi_{d}, \gamma, d, \theta, x, \Delta$ are solutions of (3.20), (3.21), (3.25), (3.26), (3.59), (3.66), then

$$
\pi-\phi_{d}, \quad \pi-\gamma, \quad d, \quad \pi-\theta, \quad x, \quad-\Delta
$$

are solutions as well. Consequently, (3.68) allows us to go easily from the $\phi_{d} \sim 47^{\circ}$ to the $\phi_{d} \sim 133^{\circ}$ case. Interestingly, for the value of $\theta$ in (3.41), we obtain $\cos \theta \sim-0.7<0$, exhibiting the same sign as within factorization, where $\left.\theta\right|_{\text {fact }}=180^{\circ}$. On the other hand, the value of $\theta$ corresponding to $\phi_{d} \sim 133^{\circ}$ yields $\cos \theta \sim+0.7>0$, i.e. the opposite sign, thereby disfavouring the $\phi_{d} \sim 133^{\circ}$ solution. Moreover, also the experimental 


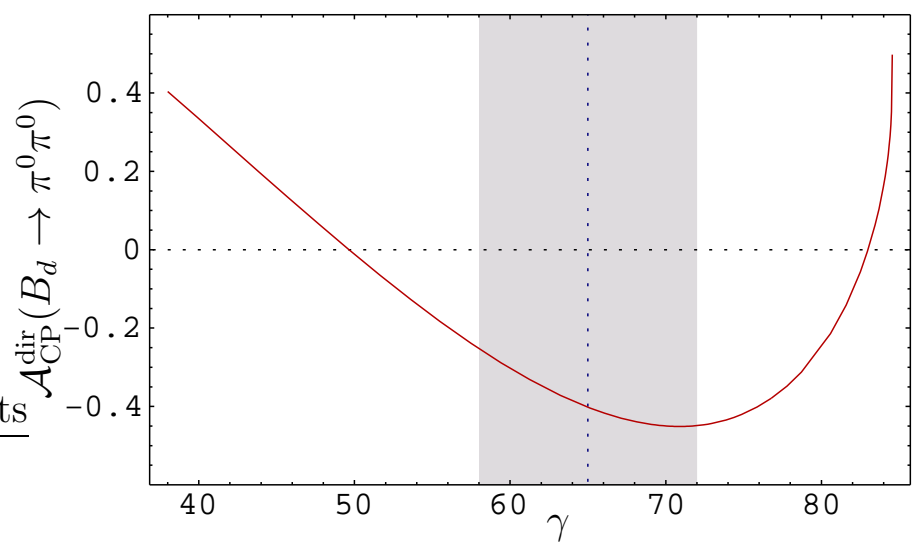

Figure 5: The dependence of $\mathcal{A}_{\mathrm{CP}}^{\text {dir }}\left(B_{d} \rightarrow \pi^{0} \pi^{0}\right)$ on $\gamma$, as described in the text.

sign information about $\mathrm{CP}$ violation in $B_{d} \rightarrow D^{(*) \pm} \pi^{\mp}$ modes, obtained recently by the BaBar collaboration, points towards $\phi_{d} \sim 47^{\circ}[75]$. Unfortunately, the Belle collaboration favours the opposite sign [11].

We shall return to $\mathcal{A}_{\mathrm{CP}}^{\mathrm{dir}}\left(B_{d} \rightarrow \pi^{\mp} K^{ \pm}\right)$in Subsection 4.4.1, and to the determination of $\gamma$ in Subsection 4.3, where we shall also address the other two angles, $\alpha$ and $\beta$, of the UT. For the following numerical analyses, we will continue to use $\gamma$ as given in (3.31).

\subsection{Prediction of CP Violation in $B_{d} \rightarrow \pi^{0} \pi^{0}$}

Let us consider, as the last element of our $B \rightarrow \pi \pi$ analysis, the $\mathrm{CP}$-violating observables of the decay $B_{d} \rightarrow \pi^{0} \pi^{0}$, which is - in analogy to $B_{d} \rightarrow \pi^{+} \pi^{-}-$another transition into a CP-even final state. If we apply the standard formalism as discussed for $B_{d} \rightarrow \pi^{+} \pi^{-}$ in $(3.22)-(3.24)$, we obtain

$$
\begin{gathered}
\mathcal{A}_{\mathrm{CP}}^{\mathrm{dir}}\left(B_{d} \rightarrow \pi^{0} \pi^{0}\right)=\frac{2 d x \sin (\theta-\Delta) \sin \gamma}{d^{2}+2 d x \cos (\theta-\Delta) \cos \gamma+x^{2}} \\
\mathcal{A}_{\mathrm{CP}}^{\operatorname{mix}}\left(B_{d} \rightarrow \pi^{0} \pi^{0}\right)=\frac{d^{2} \sin \phi_{d}+2 d x \cos (\theta-\Delta) \sin \left(\phi_{d}+\gamma\right)+x^{2} \sin \left(\phi_{d}+2 \gamma\right)}{d^{2}+2 d x \cos (\theta-\Delta) \cos \gamma+x^{2}} .
\end{gathered}
$$

Complementing these formulae with the analysis outlined above, we may predict these CP-violating observables, with the following SM result:

$$
\begin{aligned}
& \left.\mathcal{A}_{\mathrm{CP}}^{\mathrm{dir}}\left(B_{d} \rightarrow \pi^{0} \pi^{0}\right)\right|_{\mathrm{SM}}=-0.41_{-0.17}^{+0.35} \\
& \left.\mathcal{A}_{\mathrm{CP}}^{\mathrm{mix}}\left(B_{d} \rightarrow \pi^{0} \pi^{0}\right)\right|_{\mathrm{SM}}=-0.55_{-0.45}^{+0.43},
\end{aligned}
$$

where the errors come from the procedure described in Appendix C. In the future, when the errors of our input quantities will decrease, more accurate predictions for these quantities will be possible.

It is interesting to note that a measurement of one of the $\mathrm{CP}$ asymmetries of the $B_{d} \rightarrow \pi^{0} \pi^{0}$ mode would allow an essentially clean determination of $\gamma$. In Figs. 5 and 


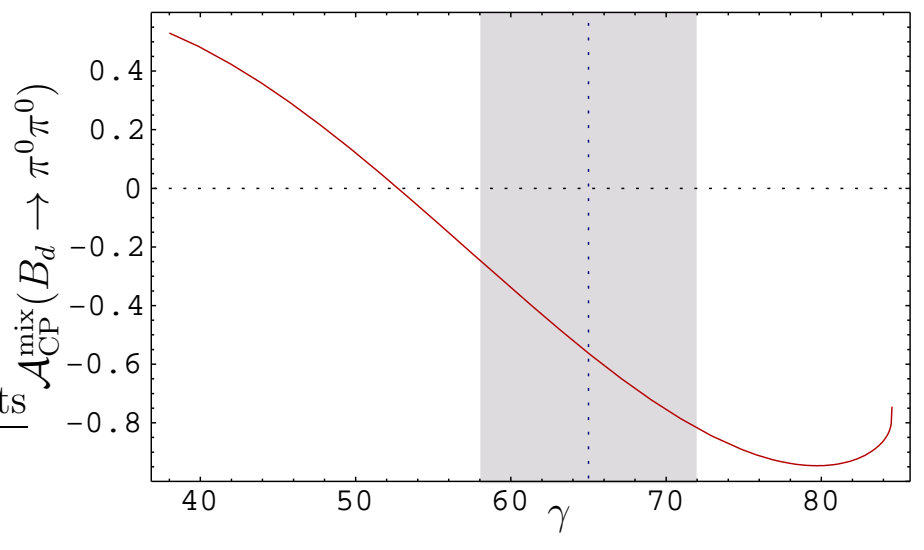

Figure 6: The dependence of $\mathcal{A}_{\mathrm{CP}}^{\mathrm{mix}}\left(B_{d} \rightarrow \pi^{0} \pi^{0}\right)$ on $\gamma$, as described in the text.

6, we illustrate the dependences of $\mathcal{A}_{\mathrm{CP}}^{\mathrm{dir}}\left(B_{d} \rightarrow \pi^{0} \pi^{0}\right)$ and $\mathcal{A}_{\mathrm{CP}}^{\operatorname{mix}}\left(B_{d} \rightarrow \pi^{0} \pi^{0}\right)$ on this angle, respectively, where we consider the central experimental values of $R_{+-}^{\pi \pi}, R_{00}^{\pi \pi}$, $\mathcal{A}_{\mathrm{CP}}^{\mathrm{dir}}\left(B_{d} \rightarrow \pi^{+} \pi^{-}\right)$and $\mathcal{A}_{\mathrm{CP}}^{\mathrm{mix}}\left(B_{d} \rightarrow \pi^{+} \pi^{-}\right)$for simplicity. ${ }^{6}$ We observe that in particular $\mathcal{A}_{\mathrm{CP}}^{\text {mix }}\left(B_{d} \rightarrow \pi^{0} \pi^{0}\right)$ - being more sensitive to $\gamma$ than $\mathcal{A}_{\mathrm{CP}}^{\text {dir }}\left(B_{d} \rightarrow \pi^{0} \pi^{0}\right)$ - would allow a useful determination of this angle, which would also be unambiguous in the range of $\gamma$ considered.

\subsection{EW Penguin Contributions}

Let us finally come back to the EW penguin contributions to the $B \rightarrow \pi \pi$ modes, which we have neglected so far. Using the results of $[13,60]$, we may take them into account with the help of the isospin symmetry of strong interactions. If we consider the SM and absorb the colour-suppressed EW penguins in the amplitude $P,(3.1)-(3.3)$ are modified as follows:

$$
\begin{aligned}
\sqrt{2} A\left(B^{+} \rightarrow \pi^{+} \pi^{0}\right) & =-|\tilde{T}| e^{i \delta_{\tilde{T}}}\left[1+x e^{i \Delta}\right]\left[e^{i \gamma}+\tilde{q} e^{-i \beta}\right] \\
A\left(B_{d}^{0} \rightarrow \pi^{+} \pi^{-}\right) & =-|\tilde{T}| e^{i \delta_{\tilde{T}}}\left[e^{i \gamma}-d e^{i \theta}\right] \\
\sqrt{2} A\left(B_{d}^{0} \rightarrow \pi^{0} \pi^{0}\right) & =|P| e^{i \delta_{P}}\left[1+\frac{x}{d} e^{i \gamma} e^{i(\Delta-\theta)}+\tilde{q}\left(\frac{1+x e^{i \Delta}}{d}\right) e^{-i \theta} e^{-i \beta}\right],
\end{aligned}
$$

where

$$
\tilde{q} \equiv\left|\frac{P_{\mathrm{EW}}}{T+C}\right| \approx 1.3 \times 10^{-2} \times\left|\frac{V_{t d}}{V_{u b}}\right|=1.3 \times 10^{-2} \times\left(1-\frac{\lambda^{2}}{2}\right)\left|\frac{\sin \gamma}{\sin \beta}\right| \approx 3 \times 10^{-2} .
$$

The impact of the EW penguin contributions on the determination of the hadronic parameters $(d, \theta)$ and $(x, \Delta)$ discussed above is negligible. For $\tilde{q}$ as in (3.76), $x$ changes

\footnotetext{
${ }^{6}$ We suppress also certain unphysical branches in these figures, which are related to unphysical solutions for the hadronic parameters $d, \theta, x$ and $\Delta$.
} 
by about $1 \%$ and $\Delta$ by $1^{\circ}$, whereas $d$ and $\theta$ are not affected by $\tilde{q}$. Even if an arbitrary (NP) weak phase for $\tilde{q}$ is admitted, $x$ changes by at most $\pm 4 \%$ and $\Delta$ by $\pm 2^{\circ}$.

In contrast to the $B \rightarrow \pi \pi$ decays, there are modes in the $B \rightarrow \pi K$ system that are significantly affected by EW penguins $[58,59,76]$, since the CKM structure of these channels is very different from the $B \rightarrow \pi \pi$ case. Despite complications for the exploration of the $B \rightarrow \pi K$ system, this feature offers a nice avenue for NP to manifest itself in the $B$-factory data.

\subsection{Summary}

Before having a closer look at the $B \rightarrow \pi K$ system in the next section, let us summarize the main results of our $B \rightarrow \pi \pi$ analysis:

- The starting point is a general parametrization of the relevant decay amplitudes. Assuming that $\gamma$ and $\phi_{d}=2 \beta$ agree with the SM expectations, we may extract the hadronic parameters from the $B \rightarrow \pi \pi$ data in an essentially clean manner.

- This analysis results in values of the hadronic parameters that show large nonfactorizable contributions. In particular, we have seen that the amazingly large $B_{d} \rightarrow \pi^{0} \pi^{0}$ branching ratio and the surprisingly small $B_{d} \rightarrow \pi^{+} \pi^{-}$branching ratio can be conveniently accommodated in the SM through constructive and destructive hadronic interference effects, respectively. The $B^{ \pm} \rightarrow \pi^{ \pm} \pi^{0}$ channel, which is measured in accordance with the conventional theoretical estimates, is not affected by this mechanism. Moreover, we expect that also the $B \rightarrow \pi \pi$ colour-suppression factor is sizeably affected by non-factorizable contributions, where, for example, $a_{2}^{\pi \pi} e^{i \Delta_{2}^{\pi \pi}} \sim 0.5 \times e^{-i 70^{\circ}}$ would fit nicely into our picture.

- The $B_{d} \rightarrow K^{+} K^{-}, B_{s} \rightarrow \pi^{+} \pi^{-}$system allows us to probe the importance of penguin annihilation and exchange topologies, which are expected to play a minor rôle. This strategy is particularly promising for the era of the LHC. The current $B$-factory bounds on $B_{d} \rightarrow K^{+} K^{-}$do not indicate any anomalous behaviour.

- Assuming that penguin annihilation and exchange topologies are negligible, we may complement the $B \rightarrow \pi \pi$ data in a variety of ways with the experimental information provided by $B_{d} \rightarrow \pi^{\mp} K^{ \pm}$modes, which are only marginally affected by EW penguins. Following these lines, we may obtain insights into $S U(3)$-breaking effects and may also determine $\gamma$. Interestingly, we obtain results in good agreement with the well-known UT fits, and arrive at a remarkably consistent overall picture.

- Finally, having all relevant parameters at hand, we may predict the CP-violating observables of the decay $B_{d} \rightarrow \pi^{0} \pi^{0}$, with the promising perspective of having large direct and mixing-induced $\mathrm{CP}$ violation in this channel. A measurement of one of the corresponding CP asymmetries would allow a clean determination of $\gamma$. 


\section{The $B \rightarrow \pi K$ System}

Decays of the kind $B \rightarrow \pi K$ have received a lot of attention in the literature over the last couple of years (for reviews, see $[2,77]$ ), mainly in the context of the determination of the UT angle $\gamma$. However, as was emphasized in $[13,59,78]$, these modes offer also valuable insights into the world of EW penguins. Interestingly, puzzling features of the current $B$-factory data for the $B \rightarrow \pi K$ modes may point towards NP effects in the EW penguin sector, as we will show in this section. A crucial ingredient of this analysis is given by the hadronic $B \rightarrow \pi \pi$ parameters determined in Section 3, which emerged from our resolution of the " $B \rightarrow \pi \pi$ puzzle" within the SM.

\subsection{Basic Formulae}

If we employ the isospin flavour symmetry of strong interactions, we may decompose the $B \rightarrow \pi K$ amplitudes in the following manner:

$$
\begin{aligned}
A\left(B^{+} \rightarrow \pi^{+} K^{0}\right) & =-P^{\prime}\left[1+\rho_{\mathrm{c}} e^{i \theta_{\mathrm{c}}} e^{i \gamma}\right] \\
\sqrt{2} A\left(B^{+} \rightarrow \pi^{0} K^{+}\right) & =P^{\prime}\left[1+\rho_{\mathrm{c}} e^{i \theta_{\mathrm{c}}} e^{i \gamma}-\left(e^{i \gamma}-q e^{i \phi} e^{i \omega}\right) r_{\mathrm{c}} e^{i \delta_{\mathrm{c}}}\right] \\
A\left(B_{d}^{0} \rightarrow \pi^{-} K^{+}\right) & =P^{\prime}\left[1-r e^{i \delta} e^{i \gamma}\right] \\
\sqrt{2} A\left(B_{d}^{0} \rightarrow \pi^{0} K^{0}\right) & =-P^{\prime}\left[1+\rho_{\mathrm{n}} e^{i \theta_{\mathrm{n}}} e^{i \gamma}-q e^{i \phi} e^{i \omega} r_{\mathrm{c}} e^{i \delta_{\mathrm{c}}}\right] .
\end{aligned}
$$

Here the CP-conserving strong amplitude

$$
P^{\prime} \equiv\left(1-\frac{\lambda^{2}}{2}\right) A \lambda^{2}\left(\mathcal{P}_{t}^{\prime}-\mathcal{P}_{c}^{\prime}\right)
$$

is the $B \rightarrow \pi K$ counterpart of $(3.4),{ }^{7}$ describing the difference of the QCD penguins with internal top- and charm-quark exchanges,

$$
\rho_{\mathrm{c}} e^{i \theta_{\mathrm{c}}} \equiv\left(\frac{\lambda^{2} R_{b}}{1-\lambda^{2}}\right)\left[\frac{\mathcal{P}_{t}^{\prime}-\tilde{\mathcal{P}}_{u}^{\prime}-\mathcal{A}^{\prime}}{\mathcal{P}_{t}^{\prime}-\mathcal{P}_{c}^{\prime}}\right],
$$

where $\tilde{\mathcal{P}}_{u}^{\prime}$ is the strong amplitude of QCD penguins with internal up-quark exchanges contributing to the charged $B \rightarrow \pi K$ decays and $\mathcal{A}^{\prime}$ denotes an annihilation amplitude,

$$
r_{\mathrm{c}} e^{i \delta_{\mathrm{c}}} \equiv\left(\frac{\lambda^{2} R_{b}}{1-\lambda^{2}}\right)\left[\frac{\mathcal{T}^{\prime}+\mathcal{C}^{\prime}}{\mathcal{P}_{t}^{\prime}-\mathcal{P}_{c}^{\prime}}\right],
$$

where $\mathcal{T}^{\prime}$ and $\mathcal{C}^{\prime}$ are the colour-allowed and colour-suppressed tree-diagram-like topologies corresponding to their $B \rightarrow \pi \pi$ counterparts $\mathcal{T}$ and $\mathcal{C}$ in (3.5) and (3.6), respectively,

$$
r e^{i \delta} \equiv\left(\frac{\lambda^{2} R_{b}}{1-\lambda^{2}}\right)\left[\frac{\mathcal{T}^{\prime}-\left(\mathcal{P}_{t}^{\prime}-\mathcal{P}_{u}^{\prime}\right)}{\mathcal{P}_{t}^{\prime}-\mathcal{P}_{c}^{\prime}}\right],
$$

\footnotetext{
${ }^{7}$ The primes remind us that we are dealing with $\bar{b} \rightarrow \bar{s}$ transitions.
} 
where $\mathcal{P}_{u}^{\prime}$ is the strong amplitude of QCD penguins with internal up-quark exchanges contributing to the neutral $B \rightarrow \pi K$ decays,

$$
\rho_{\mathrm{n}} e^{i \theta_{\mathrm{n}}} \equiv\left(\frac{\lambda^{2} R_{b}}{1-\lambda^{2}}\right)\left[\frac{\mathcal{C}^{\prime}+\left(\mathcal{P}_{t}^{\prime}-\mathcal{P}_{u}^{\prime}\right)}{\mathcal{P}_{t}^{\prime}-\mathcal{P}_{c}^{\prime}}\right],
$$

and the EW penguin parameter $q e^{i \phi} e^{i \omega}$ was introduced in (2.14). In (4.1)-(4.4), we have neglected certain contributions from colour-suppressed EW penguins, which are expected to have a minor impact on our analysis. Since a detailed discussion of these topologies is rather technical, we have left it to Appendix D, where also the corresponding generalizations of (4.1)-(4.4) can be found. As we will see in Subsection 4.4, the current $B$-factory data for those $B \rightarrow \pi K$ modes where EW penguins may only contribute in colour-suppressed form do not indicate any anomalous behaviour, i.e. do not point towards an unexpected enhancement of the colour-suppressed EW penguins. The amplitudes in (4.1)-(4.4) satisfy the following well-known isospin relation [79]:

$$
\begin{gathered}
A\left(B^{+} \rightarrow \pi^{+} K^{0}\right)+\sqrt{2} A\left(B^{+} \rightarrow \pi^{0} K^{+}\right)=A\left(B_{d}^{0} \rightarrow \pi^{-} K^{+}\right)+\sqrt{2} A\left(B_{d}^{0} \rightarrow \pi^{0} K^{0}\right) \\
=-\left[e^{i \gamma}-q e^{i \phi} e^{i \omega}\right]\left|T^{\prime}+C^{\prime}\right| e^{i \delta_{T^{\prime}+C^{\prime}}}
\end{gathered}
$$

as well as [59]

$$
A\left(B^{+} \rightarrow \pi^{+} K^{0}\right)+A\left(B_{d}^{0} \rightarrow \pi^{-} K^{+}\right)=-e^{i \gamma}\left[r e^{i \delta}+\rho_{\mathrm{c}} e^{i \theta_{\mathrm{c}}}\right]\left|P^{\prime}\right| e^{i \delta_{P^{\prime}}} .
$$

Let us also note that the hadronic parameters $\rho_{\mathrm{c}} e^{i \theta_{\mathrm{c}}}, r_{\mathrm{c}} e^{i \delta_{\mathrm{c}}}, r e^{i \delta}$ and $\rho_{\mathrm{n}} e^{i \theta_{\mathrm{n}}}$ introduced above do not involve any EW penguin contributions, in contrast to the quantities appearing in the parametrization proposed in [13]. This feature is important for the following considerations.

\subsection{Determination of the Hadronic Parameters}

\subsection{1 $\rho_{\mathrm{c}} e^{i \theta_{\mathrm{c}}}$}

As can be seen in (4.6), the parameter $\rho_{\mathrm{c}} e^{i \theta_{\mathrm{c}}}$ entering the charged $B \rightarrow \pi K$ decays is expected to be tiny because of $\lambda^{2} R_{b} \sim 0.02$. On the other hand, a sizeable value of $\rho_{\mathrm{c}}$ would be indicated by a significant direct CP asymmetry

$$
\begin{aligned}
\mathcal{A}_{\mathrm{CP}}^{\operatorname{dir}}\left(B^{ \pm} \rightarrow \pi^{ \pm} K\right) \equiv & \frac{\operatorname{BR}\left(B^{+} \rightarrow \pi^{+} K^{0}\right)-\mathrm{BR}\left(B^{-} \rightarrow \pi^{-} \bar{K}^{0}\right)}{\operatorname{BR}\left(B^{+} \rightarrow \pi^{+} K^{0}\right)+\operatorname{BR}\left(B^{-} \rightarrow \pi^{-} \bar{K}^{0}\right)} \\
& =-\left[\frac{2 \rho_{\mathrm{c}} \sin \theta_{\mathrm{c}} \sin \gamma}{1+2 \rho_{\mathrm{c}} \cos \theta_{\mathrm{c}} \cos \gamma+\rho_{\mathrm{c}}^{2}}\right]
\end{aligned}
$$

and an enhancement of the $\mathrm{CP}$-averaged $B^{ \pm} \rightarrow K^{ \pm} K$ branching ratio $[62,80]$. However, the current $B$-factory results for these quantities [11],

$$
\begin{gathered}
\mathcal{A}_{\mathrm{CP}}^{\text {dir }}\left(B^{ \pm} \rightarrow \pi^{ \pm} K\right)=-0.02 \pm 0.06 \\
\operatorname{BR}\left(B^{ \pm} \rightarrow K^{ \pm} K\right)<2.4 \times 10^{-6}(90 \% \text { C.L. })
\end{gathered}
$$


do not indicate any anomalous behaviour. In particular, if we employ the $U$-spin flavour symmetry of strong interactions and introduce (for a detailed discussion, see [2])

$$
K \equiv\left[\frac{1}{\epsilon R_{S U(3)}^{2}}\right]\left[\frac{\mathrm{BR}\left(B^{ \pm} \rightarrow \pi^{ \pm} K\right)}{\mathrm{BR}\left(B^{ \pm} \rightarrow K^{ \pm} K\right)}\right]=\frac{1+2 \rho_{\mathrm{c}} \cos \theta_{\mathrm{c}} \cos \gamma+\rho_{\mathrm{c}}^{2}}{\epsilon^{2}-2 \epsilon \rho_{\mathrm{c}} \cos \theta_{\mathrm{c}} \cos \gamma+\rho_{\mathrm{c}}^{2}}
$$

we obtain the following allowed range for $\rho_{\mathrm{c}}$, which has the same structure as (3.57):

$$
\frac{1-\epsilon \sqrt{K}}{1+\sqrt{K}} \leq \rho_{\mathrm{c}} \leq \frac{1+\epsilon \sqrt{K}}{|1-\sqrt{K}|}
$$

Using now $R_{S U(3)}=0.7$, which describes factorizable $U$-spin-breaking corrections [2], and $\operatorname{BR}\left(B^{ \pm} \rightarrow K^{ \pm} K\right) / \mathrm{BR}\left(B^{ \pm} \rightarrow \pi^{ \pm} K\right)<0.1$, which follows from (4.13) and the experimental result $\mathrm{BR}\left(B^{ \pm} \rightarrow \pi^{ \pm} K\right)=(21.8 \pm 1.4) \times 10^{-6}$ [11], we arrive at

$$
\rho_{\mathrm{c}}<0.1
$$

In the future, this bound can be improved significantly. We shall neglect the parameter $\rho_{\mathrm{c}}$ in the following discussion. Should $B^{ \pm} \rightarrow K^{ \pm} K$ decays soon be observed at the $B$ factories, thereby indicating a value of $\rho_{\mathrm{c}}$ at the 0.1 level, this parameter could be taken into account by following the strategies discussed in $[13,46,62,80]$. In this context, it should be emphasized that only the analysis of the charged $B \rightarrow \pi K$ system may be affected by $\rho_{\mathrm{c}}$, whereas this quantity does not affect the neutral $B \rightarrow \pi K$ decays.

\subsection{2 $r_{\mathrm{c}} e^{i \delta_{\mathrm{c}}}, r e^{i \delta}$ and $\rho_{\mathrm{n}} e^{i \theta_{\mathrm{n}}}$}

Let us now turn to the other hadronic parameters $r_{\mathrm{c}} e^{i \delta_{\mathrm{c}}}, r e^{i \delta}$ and $\rho_{\mathrm{n}} e^{i \theta_{\mathrm{n}}}$ appearing in (4.1)-(4.4). If we look at (4.7), (4.8) and (4.9), we may derive the following relations:

$$
\begin{gathered}
r_{\mathrm{c}} e^{i \delta_{\mathrm{c}}}=r e^{i \delta}+\rho_{\mathrm{n}} e^{i \theta_{\mathrm{n}}} \\
\rho_{\mathrm{n}} e^{i \theta_{\mathrm{n}}}=r e^{i \delta} x^{\prime} e^{i \Delta^{\prime}}
\end{gathered}
$$

with

$$
x^{\prime} e^{i \Delta^{\prime}} \equiv \frac{\mathcal{C}^{\prime}+\mathcal{P}_{t u}^{\prime}}{\mathcal{T}^{\prime}-\mathcal{P}_{t u}^{\prime}}
$$

Consequently, $r_{\mathrm{c}} e^{i \delta_{\mathrm{c}}}$ and $r e^{i \delta}$ differ through the quantity $\rho_{\mathrm{n}} e^{i \theta_{\mathrm{n}}}$, which is proportional to $x^{\prime} e^{i \Delta^{\prime}}$. Let us next assume that the penguin annihilation and exchange topologies discussed in Subsection 3.3.2 play a minor rôle. Using then the $S U(3)$ flavour symmetry of strong interactions, we may relate the hadronic $B \rightarrow \pi K$ parameter $x^{\prime} e^{i \Delta^{\prime}}$ to its $B \rightarrow \pi \pi$ counterpart $x e^{i \Delta}$ introduced in (3.14) through the simple relation

$$
x^{\prime} e^{i \Delta^{\prime}}=x e^{i \Delta}
$$

As far as the parameter $r e^{i \delta}$ is concerned, we have

$$
r e^{i \delta}=\frac{\epsilon}{d} e^{i(\pi-\theta)}
$$


with $\epsilon$ defined in (3.56). Consequently, (4.17), (4.18), (4.20) and (4.21) allow us to determine $r e^{i \delta}, \rho_{\mathrm{n}} e^{i \theta_{\mathrm{n}}}$ and $r_{\mathrm{c}} e^{i \delta_{\mathrm{c}}}$ from the $B \rightarrow \pi \pi$ analysis performed in Section 3. Following these lines, the numerical values given in (3.41) and (3.42) imply

$$
\begin{array}{cc}
r=0.11_{-0.05}^{+0.07}, & \delta=+\left(42_{-19}^{+23}\right)^{\circ} \\
\rho_{\mathrm{n}}=0.13_{-0.05}^{+0.07}, & \theta_{\mathrm{n}}=-\left(29_{-26}^{+21}\right)^{\circ} \\
r_{\mathrm{c}}=0.20_{-0.07}^{+0.09}, & \delta_{\mathrm{c}}=+\left(2_{-18}^{+23}\right)^{\circ},
\end{array}
$$

where our treatment of errors is described in Appendix C. Interestingly, the values in (4.24) mimic the picture of QCD factorization [9, 81], whereas (4.22) and (4.23) differ strongly from the corresponding predictions.

The parameter $r_{\mathrm{c}}$ in (4.24), which follows directly from the simple expression

$$
r_{\mathrm{c}}=\frac{\epsilon}{d} \sqrt{\left[1-2 d \cos \theta \cos \gamma+d^{2}\right] R_{+-}^{\pi \pi}},
$$

can be determined alternatively with the help of the following well-known relation [82]:

$$
r_{\mathrm{c}}=\sqrt{2}\left|\frac{V_{u s}}{V_{u d}}\right| \frac{f_{K}}{f_{\pi}} \sqrt{\frac{\mathrm{BR}\left(B^{ \pm} \rightarrow \pi^{ \pm} \pi^{0}\right)}{\mathrm{BR}\left(B^{ \pm} \rightarrow \pi^{ \pm} K^{0}\right)}}=0.196 \pm 0.016,
$$

which relies on the $S U(3)$ flavour symmetry and the neglect of the $\rho_{\mathrm{c}}$ term in (4.1). We consider the agreement between the numerical values in (4.24) and (4.26) as very remarkable. In particular, (4.24) does not rely on any assumption about the $\rho_{\mathrm{c}}$ parameter, thereby supporting its neglect, in addition to the arguments given above. Whereas the impact of $\rho_{\mathrm{c}}$ on (4.26) is maximal for $\theta_{\mathrm{c}} \sim 0^{\circ} \vee 180^{\circ}$, as this strong phase enters there through $\cos \theta_{\mathrm{c}},(4.11)$ is governed by $\sin \theta_{\mathrm{c}}$, and is hence affected most for $\theta_{\mathrm{c}} \sim \pm 90^{\circ}$. Consequently, we obtain complementary information on $\rho_{\mathrm{c}}$, suggesting that this parameter is indeed negligible. Interestingly, also enhanced colour-suppressed EW penguins (see Appendix D) could affect the determination of $r_{\mathrm{c}}$ through (4.26), thereby leading to a possible discrepancy with the value following from (4.25). The agreement between these two determinations of $r_{\mathrm{c}}$ does therefore also not point towards an anomalous behaviour of the colour-suppressed EW penguins. Moreover, it suggests moderate non-factorizable $S U(3)$-breaking corrections. ${ }^{8}$ A comprehensive study of the $S U(3)$-breaking corrections to the relation in (4.26) was recently performed with the help of QCD sum rules [83], with the result that the dominant effects are actually described by the "factorizable" $f_{K} / f_{\pi}$ factor, which is in accordance with the picture following from our phenomenological analysis. In this context, it is also interesting to note that (4.21) is not affected by any $S U(3)$-breaking corrections within the factorization approximation, as the corresponding decay constants and form factors cancel [53]. On the other hand, (4.20) is affected by factorizable $S U(3)$-breaking corrections in the following manner:

$$
x^{\prime} e^{i \Delta^{\prime}}=\left[\frac{f_{\pi} F_{B K}\left(M_{\pi}^{2} ; 0^{+}\right)}{f_{K} F_{B \pi}\left(M_{K}^{2} ; 0^{+}\right)}\right] x e^{i \Delta},
$$

\footnotetext{
${ }^{8}$ Otherwise, these effects have to conspire in a very contrived way with the $\rho_{\mathrm{c}} e^{i \theta_{\mathrm{c}}}$ parameter and the colour-suppressed EW penguins, which does not seem plausible to us.
} 
where the Bauer-Stech-Wirbel model [84] points to a small deviation from 1 of the correction factor, which we shall neglect in the following discussion. These $S U(3)$-breaking effects are neglected in (4.26) as well.

\subsubsection{Further Theoretical Aspects of $\rho_{\mathrm{c}} e^{i \theta_{\mathrm{c}}}$}

From the theoretical point of view, it is instructive to complement the considerations of Subsection 3.3.1 with our $B \rightarrow \pi K$ results, and to explore the implications for $\rho_{\mathrm{c}} e^{i \theta_{\mathrm{c}}}$. If we employ (3.46) and the relations given above, we obtain

$$
\begin{aligned}
\rho_{\mathrm{c}} e^{i \theta_{\mathrm{c}}} & =\left[\left(\frac{\mathcal{P}_{t}^{\prime}-\tilde{\mathcal{P}}_{u}^{\prime}}{\mathcal{P}_{t}^{\prime}-\mathcal{P}_{u}^{\prime}}\right)-\left(\frac{\mathcal{A}^{\prime}}{\mathcal{P}_{t}^{\prime}-\mathcal{P}_{u}^{\prime}}\right)\right]\left[\frac{\zeta e^{i \Delta_{\zeta}}}{1-\zeta e^{i \Delta_{\zeta}}}\right] r e^{i \delta} \\
& \sim\left[\left(\frac{\mathcal{P}_{t}^{\prime}-\tilde{\mathcal{P}}_{u}^{\prime}}{\mathcal{P}_{t}^{\prime}-\mathcal{P}_{u}^{\prime}}\right)-\left(\frac{\mathcal{A}^{\prime}}{\mathcal{P}_{t}^{\prime}-\mathcal{P}_{u}^{\prime}}\right)\right] \tilde{\zeta} e^{i \Delta_{\tilde{\zeta}} \times 0.1 \times e^{i 42^{\circ}},}
\end{aligned}
$$

where we have used the parameter $\tilde{\zeta} e^{i \Delta_{\tilde{\zeta}}}$ introduced in (3.48) and have taken the numerical values in (4.22) into account. As can be seen in Fig. 4, $\tilde{\zeta}$ may - in principle - be as large as $\mathcal{O}(2)$. However, for values of $a_{2}^{\pi \pi} e^{i \Delta_{2}^{\pi \pi}} \sim 0.5 \times e^{-i 70^{\circ}}$, which fit nicely into the picture developed in Subsection 3.3.1, we obtain $\tilde{\zeta} e^{i \Delta_{\tilde{\zeta}}} \sim 0.57 \times e^{-i 50^{\circ}}$, thereby suppressing $\rho_{\mathrm{c}}$ to a sufficient extent. Interestingly, $\Delta_{\tilde{\zeta}}$ would then largely cancel $\delta$ in (4.28). Moreover, also the first term in square brackets on the right-hand side of this equation may suppress $\rho_{\mathrm{c}}$ even further.

\subsection{Refined Determination of $\gamma$}

Imposing the constraint that the values of $r_{\mathrm{c}}$ following from (4.25) and (4.26) agree with each other allows us also to refine the determination of $\gamma$ discussed in Subsection 3.4. In particular, it allows us to lift the degeneracy of the two solutions for $\gamma$ given in (3.64). In order to illustrate this feature, we show in Fig. 7 how the $\chi^{2}$ of a fit of the hadronic parameters to $\mathcal{A}_{\mathrm{CP}}^{\mathrm{dir}}\left(B_{d} \rightarrow \pi^{+} \pi^{-}\right), \mathcal{A}_{\mathrm{CP}}^{\operatorname{mix}}\left(B_{d} \rightarrow \pi^{+} \pi^{-}\right)$and $H$ with and without a simultaneous fit to $r_{\mathrm{c}}$ varies as a function of $\gamma$. These fits are performed by fixing $\gamma$ and then fitting the hadronic parameters for each value of $\gamma$. We observe that the inclusion of the constraint from $r_{\mathrm{c}}$ does actually lift the degeneracy, leaving us with

$$
\gamma=\left(64.7_{-6.9}^{+6.3}\right)^{\circ}
$$

The fact that the curve corresponding to the fit including $r_{\mathrm{c}}$ has its minimum in one of the minima of the other curve and practically touches the $x$-axis is non-trivial, but yet another sign of the consistency of our approach.

Using $R_{b}$ as given in (2.19) and the result for $\gamma$ in (4.29), the simple relations

$$
\bar{\rho}=R_{b} \cos \gamma, \quad \bar{\eta}=R_{b} \sin \gamma,
$$

which follow from the unitarity of the CKM matrix, allow us to calculate straightforwardly the other two angles $\alpha$ and $\beta$ of the UT, where we obtain

$$
\alpha=\left(93.6_{-9.1}^{+10.3}\right)^{\circ}, \quad \beta=\left(21.7_{-2.6}^{+2.5}\right)^{\circ} .
$$




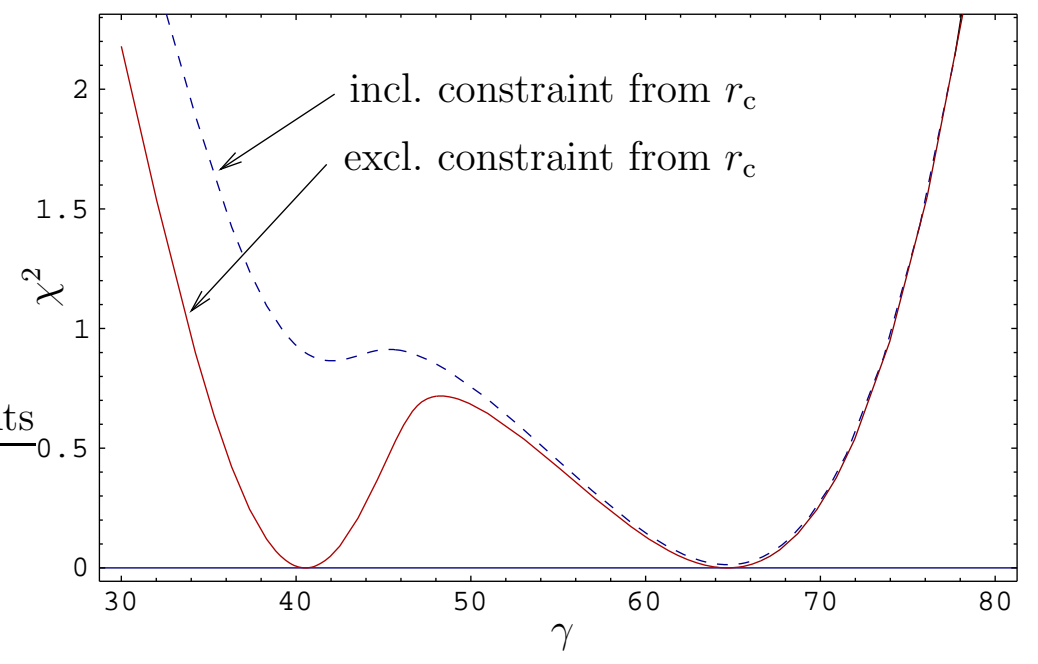

Figure 7: $\chi^{2}$ of a fit of the hadronic parameters to $\mathcal{A}_{\mathrm{CP}}^{\mathrm{dir}}\left(B_{d} \rightarrow \pi^{+} \pi^{-}\right), \mathcal{A}_{\mathrm{CP}}^{\operatorname{mix}}\left(B_{d} \rightarrow \pi^{+} \pi^{-}\right)$ and $H$ with (dashed) and without (solid) a simultaneous fit to $r_{\mathrm{c}}$ as a function of $\gamma$. By including the constraint from $r_{\mathrm{c}}$, the degeneracy is lifted.

In Fig. 8, we follow [56], and compare these results with the allowed region for the apex of the UT following from the "standard analysis" [85]. ${ }^{9}$ The solid window corresponds to the range for $\gamma$ in (4.29), whereas the dashed window indicates how the results change when the new Belle data (see Appendix B) are used. It should be noted that we show $1 \sigma$ regions, while the elliptic areas in the original UT plot show 95\% C.L. contours. Needless to note, the consistency of the overall picture is very remarkable.

\subsection{Analysis of the $B \rightarrow \pi K$ Observables}

Having all relevant hadronic parameters at hand, we may now analyse the observables provided by the $B \rightarrow \pi K$ modes within the SM, and may search for discrepancies that may shed light on NP.

\subsubsection{The $B_{d} \rightarrow \pi^{\mp} K^{ \pm}, B^{ \pm} \rightarrow \pi^{ \pm} K$ System}

Let us first turn to the decays $B_{d} \rightarrow \pi^{\mp} K^{ \pm}$and $B^{ \pm} \rightarrow \pi^{ \pm} K$. The characteristic feature of these modes is that EW penguins may only contribute in colour-suppressed form, as discussed in detail in Appendix D. Consequently, EW penguins are expected to have a marginal impact on the $B_{d} \rightarrow \pi^{\mp} K^{ \pm}, B^{ \pm} \rightarrow \pi^{ \pm} K$ system. The relevant observables are the ratio $R$ introduced in (1.5), which involves the CP-averaged branching ratios, and the corresponding direct $\mathrm{CP}$ asymmetries. If we assume again that $\rho_{\mathrm{c}}=0,(4.1)$ and (4.3) imply the following well-known expression [15]:

$$
R=1-2 r \cos \delta \cos \gamma+r^{2} .
$$

\footnotetext{
${ }^{9}$ The small and large ellipses in Fig. 8 correspond to the SM and MFV, respectively, as obtained in an update [3] of [85].
} 


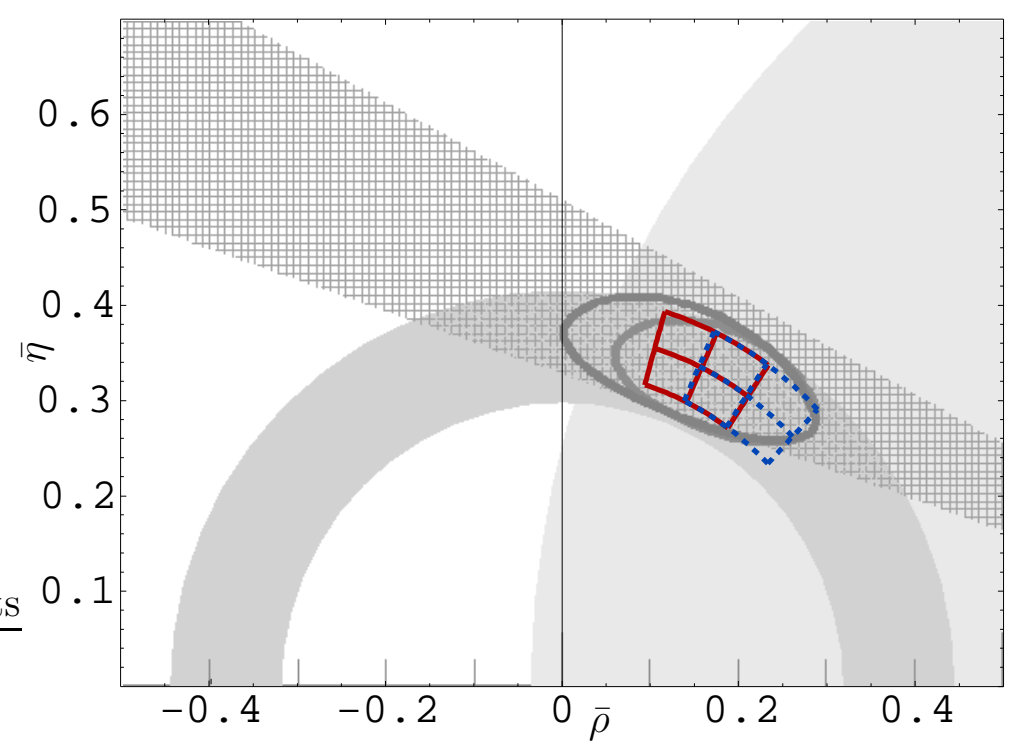

Figure 8: Comparison of our determination of $\gamma$ from the $B \rightarrow \pi \pi, \pi K$ data with the standard UT fit, where our $1 \sigma$ results are indicated by the solid and dashed windows, as described in the text.

Using the SM value of $\gamma$ in (3.31) and the hadronic parameters given in (4.22), we obtain

$$
\left.R\right|_{\mathrm{SM}}=0.943_{-0.026}^{+0.033},
$$

which agrees well with the experimental result in (1.5).

Additional information is provided by the $B_{d} \rightarrow \pi^{\mp} K^{ \pm}, B^{ \pm} \rightarrow \pi^{ \pm} K$ system through direct $\mathrm{CP}$ violation. As we have seen in (4.11), the CP asymmetry $\mathcal{A}_{\mathrm{CP}}^{\text {dir }}\left(B^{ \pm} \rightarrow \pi^{ \pm} K\right)$ vanishes for $\rho_{\mathrm{c}}=0$, in accordance with the average of the current $B$-factory data given in (4.12). Consequently, we are left with the direct CP asymmetry of the $B_{d} \rightarrow \pi^{\mp} K^{ \pm}$ modes, which we addressed already in (3.65) and (3.66). In terms of $r$ and $\delta$, this observable is given by

$$
\mathcal{A}_{\mathrm{CP}}^{\mathrm{dir}}\left(B_{d} \rightarrow \pi^{\mp} K^{ \pm}\right)=\frac{2 r \sin \delta \sin \gamma}{1-2 r \cos \delta \cos \gamma+r^{2}} .
$$

If we employ again - as in (4.33) - the SM expectation for $\gamma$ in (3.31) and the values of $r$ and $\delta$ in (4.22), this expression yields the prediction

$$
\left.\mathcal{A}_{\mathrm{CP}}^{\mathrm{dir}}\left(B_{d} \rightarrow \pi^{\mp} K^{ \pm}\right)\right|_{\mathrm{SM}}=0.140_{-0.087}^{+0.139},
$$

which is in accordance with the current $B$-factory average given in (3.65). Although we find that $r e^{i \delta}$ is strongly affected by non-factorizable effects, which is in particular reflected by the sizeable strong phase $\delta$ in (4.22), the rather small value of $r$ suppresses the direct $B_{d} \rightarrow \pi^{\mp} K^{ \pm} \mathrm{CP}$ asymmetry, in agreement with the data.

To conclude our analysis of the $B_{d} \rightarrow \pi^{\mp} K^{ \pm}, B^{ \pm} \rightarrow \pi^{ \pm} K$ system, we emphasize that the corresponding $B$-factory measurements give a current picture that does not 
show any anomalous behaviour and is nicely consistent with the SM description. From this feature, we may in particular conclude that no anomalous enhancement of coloursuppressed EW penguins is indicated by the data. Further strategies to address these topologies are discussed in Appendix D.

\subsubsection{The Charged and Neutral $B \rightarrow \pi K$ Systems}

Let us now analyse the data provided by the charged and neutral $B \rightarrow \pi K$ decays, where the quantities $R_{\mathrm{c}}$ and $R_{\mathrm{n}}$ introduced in (1.3) and (1.4), respectively, play a key rôle. In analogy to $R$, they involve CP-averaged branching ratios. Using (4.1)-(4.4) with $\rho_{\mathrm{c}}=0$, we obtain

$$
\begin{aligned}
R_{\mathrm{c}}= & 1-2 r_{\mathrm{c}} \cos \delta_{\mathrm{c}} \cos \gamma+r_{\mathrm{c}}^{2} \\
& +q r_{\mathrm{c}}\left[2\left\{\cos \left(\delta_{\mathrm{c}}+\omega\right) \cos \phi-r_{\mathrm{c}} \cos \omega \cos (\gamma-\phi)\right\}+q r_{\mathrm{c}}\right],
\end{aligned}
$$

whereas

$$
R_{\mathrm{n}}=\frac{1}{b}\left[1-2 r \cos \delta \cos \gamma+r^{2}\right]
$$

with

$$
\begin{aligned}
b= & 1-2 q r_{\mathrm{c}} \cos \left(\delta_{\mathrm{c}}+\omega\right) \cos \phi+q^{2} r_{\mathrm{c}}^{2} \\
& +2 \rho_{\mathrm{n}}\left[\cos \theta_{\mathrm{n}} \cos \gamma-q r_{\mathrm{c}} \cos \left(\theta_{\mathrm{n}}-\delta_{\mathrm{c}}-\omega\right) \cos (\gamma-\phi)\right]+\rho_{\mathrm{n}}^{2} .
\end{aligned}
$$

The quantity $b$ was introduced in [16] through

$$
b \equiv \frac{R}{R_{\mathrm{n}}}=2\left[\frac{\mathrm{BR}\left(B_{d}^{0} \rightarrow \pi^{0} K^{0}\right)+\mathrm{BR}\left(\bar{B}_{d}^{0} \rightarrow \pi^{0} \bar{K}^{0}\right)}{\mathrm{BR}\left(B^{+} \rightarrow \pi^{+} K^{0}\right)+\mathrm{BR}\left(B^{-} \rightarrow \pi^{-} \bar{K}^{0}\right)}\right] \frac{\tau_{B^{+}}}{\tau_{B_{d}^{0}}}=1.19 \pm 0.16
$$

where the experimental value follows from [11]. This variable coincides with $R_{00}$ in [9]. In contrast to (4.32), (4.36) and (4.37) are significantly affected by the EW penguin parameter $q e^{i \phi} e^{i \omega}$. Using the SM result

$$
\left.q e^{i \phi} e^{i \omega}\right|_{\mathrm{SM}}=0.69 \times\left[\frac{0.086}{\left|V_{u b} / V_{c b}\right|}\right]
$$

with $\left|V_{u b} / V_{c b}\right|$ given in (2.19) and the SM value of $\gamma$ in (3.31), the hadronic parameters in (4.22)-(4.24) yield

$$
\left.R_{\mathrm{c}}\right|_{\mathrm{SM}}=1.14_{-0.07}^{+0.08}
$$

and

$$
\left.R_{\mathrm{n}}\right|_{\mathrm{SM}}=1.11_{-0.07}^{+0.06}
$$

exhibiting a pattern that is not in accordance with the current experimental picture given in (1.3) and (1.4), respectively.

In this context, it is also interesting to consider the quantity

$$
L \equiv \frac{\left(R_{\mathrm{c}}-1\right)+b\left(1-R_{\mathrm{n}}\right)}{2 r_{\mathrm{c}}^{2}}=5.9 \pm 2.8,
$$


which was introduced in [16] and measures the violation of the Lipkin sum rule [86]; the numerical value in this expression corresponds to (4.26) and the averages of the $B$-factory data compiled in [11]. Using (4.36) and (4.37) with (4.38), we arrive at

$$
\begin{aligned}
r_{\mathrm{c}}^{2} L= & q r_{\mathrm{c}}\left[q r_{\mathrm{c}}-\left\{r_{\mathrm{c}} \cos \omega+\rho_{\mathrm{n}} \cos \left(\theta_{\mathrm{n}}-\delta_{\mathrm{c}}-\omega\right)\right\} \cos (\gamma-\phi)\right] \\
& +\left[r \cos \delta-r_{\mathrm{c}} \cos \delta_{\mathrm{c}}\right] \cos \gamma+\rho_{\mathrm{n}} \cos \theta_{\mathrm{n}} \cos \gamma+\frac{1}{2}\left(r_{\mathrm{c}}^{2}-r^{2}+\rho_{\mathrm{n}}^{2}\right) .
\end{aligned}
$$

Taking, moreover, (4.17) into account, this expression can be simplified as follows:

$$
\begin{aligned}
L= & q[q-\cos \omega \cos (\gamma-\phi)] \\
& +\frac{\rho_{\mathrm{n}}}{r_{\mathrm{c}}}\left[\left\{\frac{\rho_{\mathrm{n}}+r \cos \left(\delta-\theta_{\mathrm{n}}\right)}{r_{\mathrm{c}}}\right\}-q \cos \left(\theta_{\mathrm{n}}-\delta_{\mathrm{c}}-\omega\right) \cos (\gamma-\phi)\right] .
\end{aligned}
$$

In analogy to (4.41) and (4.42), we may then calculate

$$
\left.L\right|_{\mathrm{SM}}=0.59_{-0.13}^{+0.14}
$$

which is in conflict with the experimental number in (4.43).

Already back in 2000, when the observation of the $B_{d}^{0} \rightarrow \pi^{0} K^{0}$ channel was announced by the CLEO collaboration, two of us pointed out puzzling features that were indicated by the corresponding results for $R_{\mathrm{n}}$ and $R_{\mathrm{c}}$, emphasizing the possibility of having NP in the EW penguin sector [14]. Now we have a much better experimental picture, where the BaBar, Belle and CLEO data show the same pattern for the observables $R_{\mathrm{n}}$ and $R_{\mathrm{c}}$. In a recent paper [16], we showed that enhanced EW penguins may in fact provide a solution to this puzzle. Here we perform a considerably more refined analysis and go beyond our previous study in the following respects:

- We fix the relevant hadronic parameters $r e^{i \delta}, r_{\mathrm{c}} e^{i \delta_{\mathrm{c}}}$ and $\rho_{\mathrm{n}} e^{i \theta_{\mathrm{n}}}$ through the $B \rightarrow \pi \pi$ analysis, as discussed above. In particular, we take also $\rho_{\mathrm{n}} e^{i \theta_{\mathrm{n}}}$ into account, which is found to be sizeable because of the very recent data for the $B_{d} \rightarrow \pi^{0} \pi^{0}$ channel.

- In addition to an enhancement of $q$, we also allow for a CP-violating NP phase $\phi$ in the EW penguin sector, and for a CP-conserving strong phase $\omega$, which could be induced by large non-factorizable $S U(3)$-breaking corrections.

In order to make our analysis more transparent, let us first assume that $\omega=0^{\circ}$, as implied by the $S U(3)$ flavour symmetry [13, 45, 48]. We are then left with the two EW penguin parameters $q$ and $\phi$. In Fig. 9, we follow [16] and consider the $R_{\mathrm{n}}-R_{\mathrm{c}}$ plane for the central values of the hadronic parameters in (4.22)-(4.24) and $\gamma=65^{\circ}$, showing contours for different values of $q$ and $\phi \in\left[0^{\circ}, 360^{\circ}\right]$. We observe that we may in fact move to the experimental region for an enhanced value of $q \sim 1.8$ and $\phi \sim-90^{\circ}$, where in particular the large CP-violating phase is in stark contrast to the SM picture characterized by (4.40). In order to put these observations on a more quantitative level, we use the value of $\gamma$ in (3.31) and the hadronic parameters in (4.22)-(4.24), which allow us to convert the experimental results for $R_{\mathrm{c}}$ and $R_{\mathrm{n}}$ in (1.3) and (1.4), respectively, into values of $q$ and $\phi$. Following these lines, we obtain

$$
q=1.75_{-0.99}^{+1.27}, \quad \phi=-\left(85_{-14}^{+11}\right)^{\circ},
$$




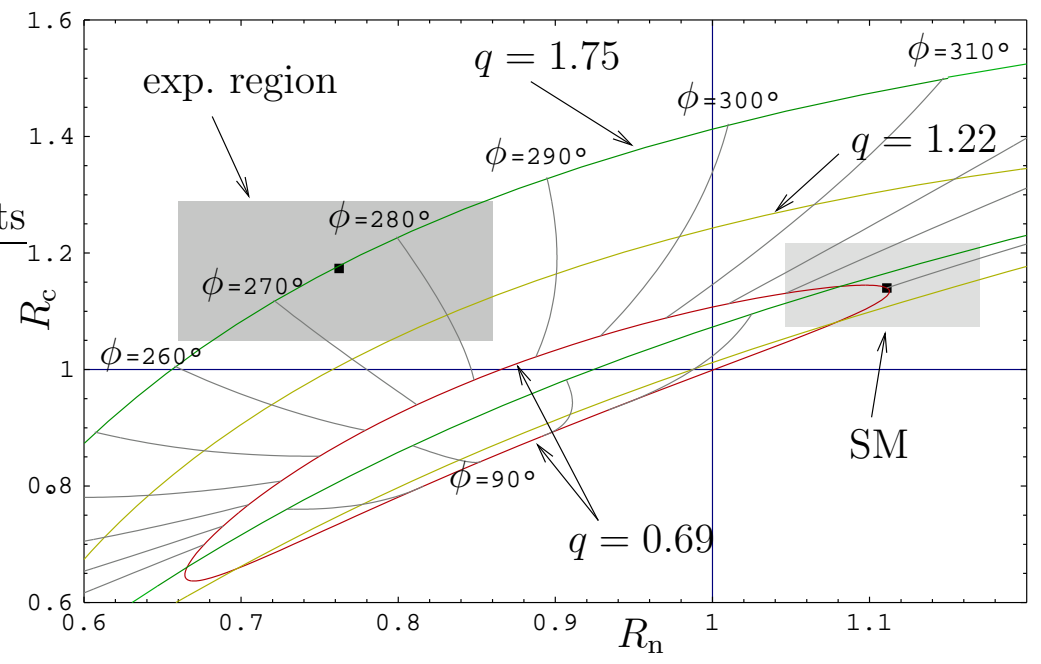

Figure 9: The situation in the $R_{\mathrm{n}}-R_{\mathrm{c}}$ plane. We show contours for values of $q=0.69$, $q=1.22$ and $q=1.75$, with $\phi \in\left[0^{\circ}, 360^{\circ}\right]$. The ranges from (1.3) and (1.4) (experiment) as well as (4.41) and (4.42) (SM) are indicated in grey.

where our treatment of errors is discussed in Appendix C. We may now also calculate the quantity $L$ in (4.45) within our NP scenario, yielding

$$
L=6.02_{-4.67}^{+7.37}
$$

in accordance with the experimental result given in (4.43).

In addition to the CP-violating asymmetries that we considered already in our analysis, there is yet another one that is strongly constrained by the $B$-factory data [11]:

$$
\mathcal{A}_{\mathrm{CP}}^{\mathrm{dir}}\left(B^{ \pm} \rightarrow \pi^{0} K^{ \pm}\right) \equiv \frac{\mathrm{BR}\left(B^{+} \rightarrow \pi^{0} K^{+}\right)-\mathrm{BR}\left(B^{-} \rightarrow \pi^{0} K^{-}\right)}{\mathrm{BR}\left(B^{+} \rightarrow \pi^{0} K^{+}\right)+\mathrm{BR}\left(B^{-} \rightarrow \pi^{0} K^{-}\right)}=0.00 \pm 0.07 .
$$

Using (4.2) with $\rho_{\mathrm{c}}=0$, we obtain

$$
\mathcal{A}_{\mathrm{CP}}^{\mathrm{dir}}\left(B^{ \pm} \rightarrow \pi^{0} K^{ \pm}\right)=\frac{2}{R_{\mathrm{c}}}\left[r_{\mathrm{c}} \sin \delta_{\mathrm{c}} \sin \gamma-q r_{\mathrm{c}}\left\{\sin \left(\delta_{\mathrm{c}}+\omega\right) \sin \phi+r_{\mathrm{c}} \sin \omega \sin (\gamma-\phi)\right\}\right],
$$

where the expression for $R_{\mathrm{c}}$ is given in (4.36). We are now in a position to calculate this $\mathrm{CP}$ asymmetry, where we obtain

$$
\left.\mathcal{A}_{\mathrm{CP}}^{\operatorname{dir}}\left(B^{ \pm} \rightarrow \pi^{0} K^{ \pm}\right)\right|_{\mathrm{SM}}=0.01_{-0.10}^{+0.14}
$$

within the SM, and

$$
\mathcal{A}_{\mathrm{CP}}^{\operatorname{dir}}\left(B^{ \pm} \rightarrow \pi^{0} K^{ \pm}\right)=0.04_{-0.28}^{+0.37}
$$

for our NP scenario, in accordance with the experimental value given in (4.49). On the other hand, the analysis performed in [16] favoured generically larger CP asymmetries. 
Consequently, the determination of the hadronic parameters through the $B \rightarrow \pi \pi$ analysis and the introduction of the CP-violating NP phase $\phi$ allow us now to achieve a much better agreement with the experimental picture.

Interestingly, we may also employ the experimental information on $R_{\mathrm{c}}, R_{\mathrm{n}}$ and the $\mathrm{CP}$ asymmetry $\mathcal{A}_{\mathrm{CP}}^{\text {dir }}\left(B^{ \pm} \rightarrow \pi^{0} K^{ \pm}\right)$to determine the three EW penguin parameters $q, \phi$ and $\omega$ simultaneously. This analysis gives

$$
q=1.74_{-0.95}^{+1.28}, \quad \phi=-\left(85_{-15}^{+11}\right)^{\circ}, \quad \omega=-\left(4_{-32}^{+44}\right)^{\circ},
$$

where a central value of $\omega$ being nicely consistent with $0^{\circ}$ - as expected in the strict $S U(3)$ limit - is very remarkable. While this feature supports also our working assumption of using the $S U(3)$ flavour symmetry, the reduction of the error on $\omega$ would be very desirable.

Let us finally note, for completeness, that there is also a mathematical solution with $\phi \sim 0^{\circ}$ and $\omega \sim 90^{\circ}$,

$$
q=2.32_{-1.04}^{+1.38}, \quad \phi=-\left(10_{-12}^{+11}\right)^{\circ}, \quad \omega=\left(82_{-28}^{+20}\right)^{\circ} .
$$

However, since such a value of $\omega$ looks completely unrealistic, we will not consider this solution further.

\subsubsection{Elimination of the Second Solution for $x e^{i \Delta}$ in (3.43)}

Let us now come back to the second solution for $x e^{i \Delta}$ represented by (3.43). Using (4.22) and the relations in (4.17)-(4.20), we obtain

$$
\begin{array}{ll}
\rho_{\mathrm{n}}=0.11_{-0.04}^{+0.05}, & \theta_{\mathrm{n}}=+\left(94_{-40}^{+36}\right)^{\circ} \\
r_{\mathrm{c}}=0.20_{-0.07}^{+0.09}, & \delta_{\mathrm{c}}=+\left(68_{-25}^{+26}\right)^{\circ},
\end{array}
$$

where the agreement between the values of $r_{\mathrm{c}}$ in (4.24) and (4.56) is obvious from (4.25). If we assume that $\omega$ vanishes, (1.3) and (1.4) give

$$
q=1.73_{-0.69}^{+1.32}, \quad \phi=-\left(102_{-29}^{+130}\right)^{\circ},
$$

yielding

$$
\mathcal{A}_{\mathrm{CP}}^{\mathrm{dir}}\left(B^{ \pm} \rightarrow \pi^{0} K^{ \pm}\right)=0.81_{-0.82}^{+0.07},
$$

which is very much disfavoured by the experimental result given in (4.49). Even the inclusion of a non-zero strong phase $\omega$ does not provide any physically attractive solution for the EW penguin parameters, implying, for instance, values for $q$ as high as 2.4 that are totally exluded by the rare-decay constraints discussed in Section 5. Consequently, we will not consider the second solution for $x e^{i \Delta}$ in (3.43) further. 


\subsection{Prediction of CP Violation in $B_{d} \rightarrow \pi^{0} K_{\mathrm{S}}$}

The decay $B_{d} \rightarrow \pi^{0} K_{\mathrm{S}}$ is a transition into a final state with CP eigenvalue -1 . Using the standard formalism for the calculation of the observables provided by the corresponding time-dependent CP asymmetry [2], as discussed for $B_{d} \rightarrow \pi^{+} \pi^{-}$in Subsection 3.1, (4.4) yields

$$
\begin{gathered}
\mathcal{A}_{\mathrm{CP}}^{\mathrm{dir}}\left(B_{d} \rightarrow \pi^{0} K_{\mathrm{S}}\right)=\frac{2}{b}\left[q r_{\mathrm{c}} \sin \left(\delta_{\mathrm{c}}+\omega\right) \sin \phi\right. \\
\left.-\rho_{\mathrm{n}}\left\{\sin \theta_{\mathrm{n}} \sin \gamma-q r_{\mathrm{c}} \sin \left(\theta_{\mathrm{n}}-\delta_{\mathrm{c}}-\omega\right) \sin (\gamma-\phi)\right\}\right] \\
\mathcal{A}_{\mathrm{CP}}^{\operatorname{mix}}\left(B_{d} \rightarrow \pi^{0} K_{\mathrm{S}}\right)=-\frac{1}{b}\left[\sin \phi_{d}-2 q r_{\mathrm{c}} \cos \left(\delta_{\mathrm{c}}+\omega\right) \sin \left(\phi_{d}+\phi\right)+q^{2} r_{\mathrm{c}}^{2} \sin \left(\phi_{d}+2 \phi\right)(4.60)\right. \\
\left.+2 \rho_{\mathrm{n}}\left\{\cos \theta_{\mathrm{n}} \sin \left(\phi_{d}+\gamma\right)-q r_{\mathrm{c}} \cos \left(\theta_{\mathrm{n}}-\delta_{\mathrm{c}}-\omega\right) \sin \left(\phi_{d}+\gamma+\phi\right)\right\}+\rho_{\mathrm{n}}^{2} \sin \left(\phi_{d}+2 \gamma\right)\right],
\end{gathered}
$$

where the expression for $b$ is given in (4.38). In the special case of $\rho_{\mathrm{n}}=0$ and $\phi=0^{\circ}$, we arrive at the following, well-known results [59]:

$$
\begin{aligned}
& \mathcal{A}_{\mathrm{CP}}^{\mathrm{dir}}\left(B_{d} \rightarrow \pi^{0} K_{\mathrm{S}}\right)=0 \\
& \mathcal{A}_{\mathrm{CP}}^{\mathrm{mix}}\left(B_{d} \rightarrow \pi^{0} K_{\mathrm{S}}\right)=-\sin \phi_{d}=\mathcal{A}_{\mathrm{CP}}^{\mathrm{mix}}\left(B_{d} \rightarrow J / \psi K_{\mathrm{S}}\right),
\end{aligned}
$$

where the average of the current $B$-factory data for the mixing-induced $B_{d} \rightarrow J / \psi K_{\mathrm{S}}$ $\mathrm{CP}$ asymmetry is given in (1.8). Recently, the BaBar collaboration reported the first results for these observables [24], which are given by

$$
\begin{aligned}
& \mathcal{A}_{\mathrm{CP}}^{\mathrm{dir}}\left(B_{d} \rightarrow \pi^{0} K_{\mathrm{S}}\right)=+0.40_{-0.28}^{+0.27} \pm 0.10 \\
& \mathcal{A}_{\mathrm{CP}}^{\mathrm{mix}}\left(B_{d} \rightarrow \pi^{0} K_{\mathrm{S}}\right)=-0.48_{-0.38}^{+0.47} \pm 0.11 .
\end{aligned}
$$

Moreover, there is also the following measurement of the direct $\mathrm{CP}$ asymmetry of the $B_{d}^{0} \rightarrow \pi^{0} K^{0}$ channel available [11]:

$$
\mathcal{A}_{\mathrm{CP}}^{\mathrm{dir}}\left(B_{d}^{0} \rightarrow \pi^{0} K^{0}\right)=-0.03 \pm 0.36 \pm 0.09
$$

which is supposed to agree with (4.63). Consequently, these experimental numbers are expected to change significantly in the future. It will also be very exciting to see the corrsponding first Belle results.

Following the analysis performed in Subsection 4.4.2, we may predict the CP-violating $B_{d} \rightarrow \pi^{0} K_{\mathrm{S}}$ observables. In order to illustrate the dependence on the EW penguin parameters, we employ - in analogy to Fig. 9 - the central values of the hadronic parameters in (4.22)-(4.24), $\omega=0^{\circ}, \gamma=65^{\circ}$, and show in Fig. 10 the contours in the $\mathcal{A}_{\mathrm{CP}}^{\mathrm{mix}}\left(B_{d} \rightarrow \pi^{0} K_{\mathrm{S}}\right)-\mathcal{A}_{\mathrm{CP}}^{\text {dir }}\left(B_{d} \rightarrow \pi^{0} K_{\mathrm{S}}\right)$ plane corresponding to various values of $q$ with $\phi \in\left[0^{\circ}, 360^{\circ}\right]$. If we take the uncertainties both of the parameters in (4.22)-(4.24) and of $\gamma$ in (3.31) into account, and assume again that $\omega=0^{\circ}$, the SM expression (4.40) yields

$$
\begin{aligned}
& \left.\mathcal{A}_{\mathrm{CP}}^{\mathrm{dir}}\left(B_{d} \rightarrow \pi^{0} K_{\mathrm{S}}\right)\right|_{\mathrm{SM}}=+0.12_{-0.13}^{+0.11} \\
& \left.\mathcal{A}_{\mathrm{CP}}^{\mathrm{mix}}\left(B_{d} \rightarrow \pi^{0} K_{\mathrm{S}}\right)\right|_{\mathrm{SM}}=-0.86_{-0.07}^{+0.05},
\end{aligned}
$$




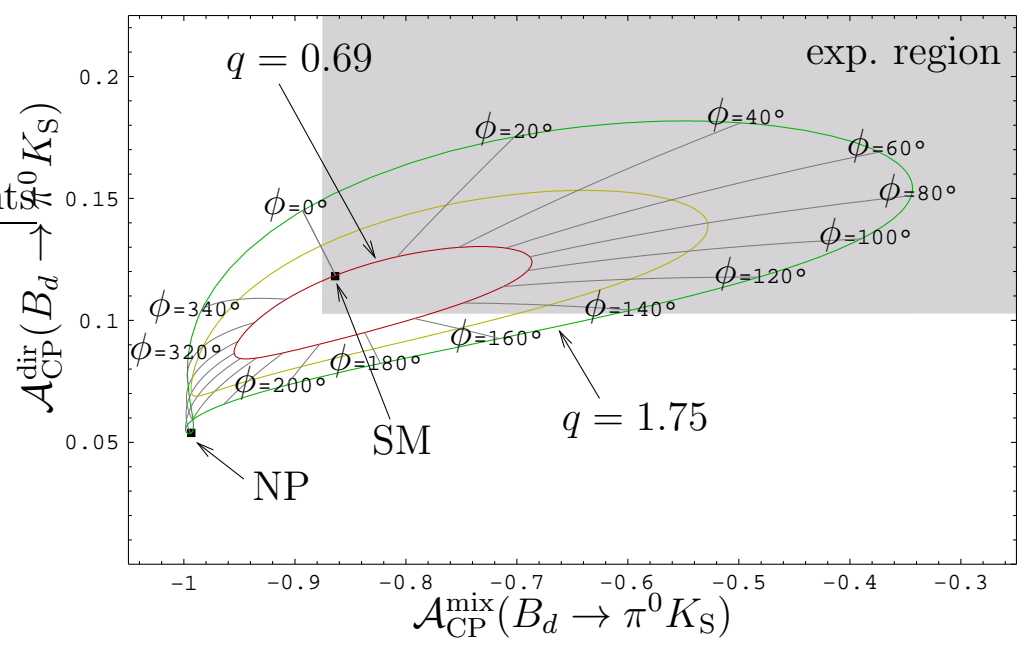

Figure 10: The situation in the $\mathcal{A}_{\mathrm{CP}}^{\text {mix }}\left(B_{d} \rightarrow \pi^{0} K_{\mathrm{S}}\right)-\mathcal{A}_{\mathrm{CP}}^{\mathrm{dir}}\left(B_{d} \rightarrow \pi^{0} K_{\mathrm{S}}\right)$ plane. We show contours for values of $q=0.69$ to $q=1.75$ and $\phi \in\left[0^{\circ}, 360^{\circ}\right]$. The grey area shows the lower left-hand corner of the $\mathrm{BaBar} 1 \sigma$ range, whilst the central value is outside the plotted area. The central values of (4.66), (4.67) and (4.68), (4.69) are indicated by the labels "SM" and "NP", respectively.

whereas our NP scenario, which is characterized by the values of $q$ and $\phi$ in (4.47), corresponds to the following prediction:

$$
\begin{aligned}
& \mathcal{A}_{\mathrm{CP}}^{\mathrm{dir}}\left(B_{d} \rightarrow \pi^{0} K_{\mathrm{S}}\right)=+0.05_{-0.29}^{+0.24} \\
& \mathcal{A}_{\mathrm{CP}}^{\text {mix }}\left(B_{d} \rightarrow \pi^{0} K_{\mathrm{S}}\right)=-0.99_{-0.01}^{+0.04} .
\end{aligned}
$$

The measurement of these CP asymmetries will allow a crucial test of our NP scenario. By the time solid experimental numbers are available, the uncertainties of the parameters entering (4.68) and (4.69) are expected to be significantly smaller, thereby leading to much more stringent predictions of $\mathrm{CP}$ violation in $B_{d} \rightarrow \pi^{0} K_{\mathrm{S}}{ }^{10}$ Constraints for these observables were also recently derived, within the framework of the SM, in [87].

\subsection{Future Avenues Offered by $B_{s}$ Decays}

The physics potential of $B_{s}$-meson decays, which can be exploited at hadronic $B$-decay experiments, i.e. at run II of the Tevatron [71] and later on at the LHC [72], provides interesting strategies to explore $\mathrm{CP}$ violation and to obtain insights into hadronic physics. These results will nicely complement the $B_{u, d} \rightarrow \pi \pi, \pi K$ methods proposed above.

\subsection{1 $\quad B_{s} \rightarrow K^{+} K^{-}$}

As pointed out in [53], the decay $B_{s} \rightarrow K^{+} K^{-}$can be related to $B_{d} \rightarrow \pi^{+} \pi^{-}$through the $U$-spin flavour symmetry of strong interactions, thereby providing attractive simultaneous determinations of $\gamma, d$ and $\theta$, as well as insights into $U$-spin-breaking effects. It will

\footnotetext{
${ }^{10} \mathrm{It}$ is evident from Fig. 10 that the error in (4.69) is accidentally small.
} 
be very exciting to see whether we will arrive at a picture that is consistent with the one developed in Section 3. Interestingly, we may use the hadronic parameters determined there to make predictions for the CP-violating $B_{s} \rightarrow K^{+} K^{-}$observables with the help of the $U$-spin flavour symmetry, which implies the following expressions:

$$
\begin{gathered}
\mathcal{A}_{\mathrm{CP}}^{\mathrm{dir}}\left(B_{s} \rightarrow K^{+} K^{-}\right)=\frac{2 \epsilon d \sin \theta \sin \gamma}{\epsilon^{2}+2 \epsilon d \cos \theta \cos \gamma+d^{2}} \\
\mathcal{A}_{\mathrm{CP}}^{\mathrm{mix}}\left(B_{s} \rightarrow K^{+} K^{-}\right)=\frac{\epsilon^{2} \sin \left(\phi_{s}+2 \gamma\right)+2 \epsilon d \cos \theta \sin \left(\phi_{s}+\gamma\right)+d^{2} \sin \phi_{s}}{\epsilon^{2}+2 \epsilon d \cos \theta \cos \gamma+d^{2}},
\end{gathered}
$$

where the $B_{s}^{0}-\bar{B}_{s}^{0}$ mixing phase $\phi_{s}$ is given by

$$
\phi_{s}=-2 \lambda^{2} \bar{\eta}=2 \beta_{s}
$$

in the SM, with the numerical value of $\beta_{s}$ in (2.20). Note that the expression for $\mathcal{A}_{\mathrm{CP}}^{\mathrm{dir}}\left(B_{s} \rightarrow K^{+} K^{-}\right)$agrees with the one for $\mathcal{A}_{\mathrm{CP}}^{\text {dir }}\left(B_{d} \rightarrow \pi^{\mp} K^{ \pm}\right)$in (3.66). Using now the range for $\gamma$ in (3.31) and the hadronic parameters in (3.41), we obtain the following SM predictions:

$$
\begin{aligned}
& \left.\mathcal{A}_{\mathrm{CP}}^{\mathrm{dir}}\left(B_{s} \rightarrow K^{+} K^{-}\right)\right|_{\mathrm{SM}}=0.14_{-0.09}^{+0.14} \\
& \left.\mathcal{A}_{\mathrm{CP}}^{\mathrm{mix}}\left(B_{s} \rightarrow K^{+} K^{-}\right)\right|_{\mathrm{SM}}=-0.18_{-0.07}^{+0.08}
\end{aligned}
$$

where the latter observable may be affected by NP contributions to $B_{s}^{0}-\bar{B}_{s}^{0}$ mixing. By the time the CP-violating $B_{s} \rightarrow K^{+} K^{-}$observables can be measured, more precise SM predictions will be available.

Moreover, we may also explore the branching ratio of this channel. To this end, we use the quantity $H$ introduced in (3.59). If we use again (3.31) and (3.41), we obtain

$$
\left.H\right|_{\mathrm{SM}}=7.0_{-4.7}^{+7.4}
$$

which is in nice accordance with the numerical value in (3.61). ${ }^{11}$ In order to be able to predict $\operatorname{BR}\left(B_{s} \rightarrow K^{+} K^{-}\right)$, we have to know the $U$-spin-breaking factor $\left|\mathcal{C}^{\prime} / \mathcal{C}\right|$, which is given - within the factorization approximation - as follows [53]:

$$
\left|\frac{\mathcal{C}^{\prime}}{\mathcal{C}}\right|_{\text {fact }}=\frac{f_{K}}{f_{\pi}} \frac{F_{B_{s} K}\left(M_{K}^{2} ; 0^{+}\right)}{F_{B_{d} \pi}\left(M_{\pi}^{2} ; 0^{+}\right)}\left(\frac{M_{B_{s}}^{2}-M_{K}^{2}}{M_{B_{d}}^{2}-M_{\pi}^{2}}\right) .
$$

In a recent analysis [83], this parameter has been calculated through QCD sum rules, with the following result:

$$
\left|\frac{\mathcal{C}^{\prime}}{\mathcal{C}}\right|_{\text {fact }}=1.76_{-0.17}^{+0.15} .
$$

If we now complement (3.59) with (4.75) and (4.77), and use the experimental result $\mathrm{BR}\left(B_{d} \rightarrow \pi^{+} \pi^{-}\right)=(4.6 \pm 0.4) \times 10^{-6}[11]$, we obtain

$$
\mathrm{BR}\left(B_{s} \rightarrow K^{+} K^{-}\right)=\left(35_{-20}^{+73}\right) \times 10^{-6} .
$$

\footnotetext{
${ }^{11}$ Because of the discussion in Subsection 3.4, this feature is of course not surprising.
} 
The very large uncertainty reflects the fact that our analysis is rather insensitive to $H$ (this can also be seen in Fig. 1), and therefore the predicted value (4.75) has a large uncertainty that propagates to (4.78).

Alternatively, we may - in the spirit of (3.61) - assume that the penguin annihilation and exchange topologies discussed in Subsection 3.3.2 play a minor rôle. Taking, moreover, factorizable $S U(3)$-breaking corrections into account, we obtain

$$
\begin{aligned}
\frac{\operatorname{BR}\left(B_{s} \rightarrow K^{+} K^{-}\right)}{\operatorname{BR}\left(B_{d} \rightarrow \pi^{\mp} K^{ \pm}\right)}= & {\left[\frac{M_{B_{d}}}{M_{B_{s}}} \frac{\Phi\left(M_{K} / M_{B_{s}}, M_{K} / M_{B_{s}}\right)}{\Phi\left(M_{\pi} / M_{B_{d}}, M_{K} / M_{B_{d}}\right)} \frac{\tau_{B_{s}^{0}}}{\tau_{B_{d}^{0}}}\right] } \\
& \times\left[\frac{F_{B_{s} K}\left(M_{K}^{2} ; 0^{+}\right)}{F_{B_{d}}\left(M_{\pi}^{2} ; 0^{+}\right)}\left(\frac{M_{B_{s}}^{2}-M_{K}^{2}}{M_{B_{d}}^{2}-M_{\pi}^{2}}\right)\right]^{2},
\end{aligned}
$$

where

$$
\frac{F_{B_{s} K}\left(M_{K}^{2} ; 0^{+}\right)}{F_{B_{d} \pi}\left(M_{\pi}^{2} ; 0^{+}\right)}\left(\frac{M_{B_{s}}^{2}-M_{K}^{2}}{M_{B_{d}}^{2}-M_{\pi}^{2}}\right)=1.45_{-0.14}^{+0.13}
$$

corresponds to (4.77) [83]. If we then use the experimental result $\mathrm{BR}\left(B_{d} \rightarrow \pi^{\mp} K^{ \pm}\right)=$ $(18.2 \pm 0.8) \times 10^{-6}[11]$, we arrive at

$$
\operatorname{BR}\left(B_{s} \rightarrow K^{+} K^{-}\right)=(35 \pm 7) \times 10^{-6}
$$

A measurement of this branching ratio, which should soon be available from run II of the Tevatron, will be very interesting, allowing in particular valuable insights into (4.77). It is interesting to note that - in contrast to (4.78) - the predictions of the CPviolating observables in (4.73) and (4.74) are not affected by factorizable $U$-spin-breaking corrections [53], i.e. do not involve a ratio of form factors as in (4.76).

\subsection{2 $\quad B_{s} \rightarrow \pi^{ \pm} K^{\mp}$}

The decay $B_{s}^{0} \rightarrow \pi^{+} K^{-}$is related to the $B_{d}^{0} \rightarrow \pi^{-} K^{+}$mode through the $U$-spin flavour symmetry of strong interactions [88], which allows us to write

$$
A\left(B_{s}^{0} \rightarrow \pi^{+} K^{-}\right)=\lambda^{3} A\left(\mathcal{P}_{t}^{\prime}-\mathcal{P}_{c}^{\prime}\right)\left[1+\frac{1}{\epsilon} r e^{i \delta} e^{i \gamma}\right]
$$

complementing the $B_{d}^{0} \rightarrow \pi^{-} K^{+}$amplitude in (4.3). If we then combine the direct CP asymmetry

$$
\mathcal{A}_{\mathrm{CP}}^{\mathrm{dir}}\left(B_{s} \rightarrow \pi^{ \pm} K^{\mp}\right)=-\left[\frac{2 \epsilon r \sin \delta \sin \gamma}{\epsilon^{2}+2 \epsilon r \cos \delta \cos \gamma+r^{2}}\right]
$$

with its $B_{d} \rightarrow \pi^{\mp} K^{ \pm}$counterpart in (4.34), we may determine $r$ and $\delta$ for given values of $\gamma \cdot{ }^{12}$ Fixing then $r_{\mathrm{c}}$ through (4.26), we may use (4.17) to eliminate $\rho_{\mathrm{n}} e^{i \theta_{\mathrm{n}}}$ in the $B_{d}^{0} \rightarrow \pi^{0} K^{0}$ amplitude, so that $R_{\mathrm{n}}$ and the two $B_{d} \rightarrow \pi^{0} K_{\mathrm{S}} \mathrm{CP}$ asymmetries depend on

\footnotetext{
${ }^{12}$ If we consider, in addition, the ratio of the CP-averaged $B_{s} \rightarrow \pi^{ \pm} K^{\mp}$ and $B^{ \pm} \rightarrow \pi^{ \pm} K$ branching ratios, we may determine $\gamma$ as well [88]. This extraction involves, however, the ratio $F_{B_{s} K}\left(M_{\pi}^{2} ; 0^{+}\right) / F_{B_{d} \pi}\left(M_{K}^{2} ; 0^{+}\right)$of $S U(3)$-breaking form factors.
} 
the strong phase $\delta_{\mathrm{c}}$ and the EW penguin parameters $q, \phi$ (and $\omega$ ). If we complement these observables with $R_{\mathrm{c}}$ and the direct $B^{ \pm} \rightarrow \pi^{0} K^{ \pm}$CP asymmetry, we may extract these parameters and may perform internal consistency checks.

The advantage of this avenue is that it is not affected by the penguin annihilation and exchange topologies discussed in Subsection 3.3.2. Consequently, it will be very interesting to see whether we will eventually arrive at a consistent overall picture. If we neglect the penguin annihilation and exchange topologies and use the $S U(3)$ flavour symmetry, we obtain the simple relation

$$
\mathcal{A}_{\mathrm{CP}}^{\mathrm{dir}}\left(B_{s} \rightarrow \pi^{ \pm} K^{\mp}\right)=\mathcal{A}_{\mathrm{CP}}^{\mathrm{dir}}\left(B_{d} \rightarrow \pi^{+} \pi^{-}\right),
$$

which should already provide important insights into this issue.

Let us finally note that EW penguins enter the $B_{s}$ modes considered above only in colour-suppressed form. In the case of $\phi=0^{\circ}$, these topologies would not affect our $B_{s}$ strategies at all, as becomes obvious from the discussion in Appendix D. On the other hand, for $\phi \neq 0^{\circ}$, anomalously enhanced colour-suppressed EW penguins would manifest themselves in the corresponding data. Consequently, the $B_{s}$ studies complement the strategies to address the colour-suppressed EW penguins discussed in Appendix D.

\subsection{Summary}

Before turning to rare $K$ and $B$ decays as well as $\varepsilon^{\prime} / \varepsilon$ in the next section, let us summarize the main results of our analysis of the $B \rightarrow \pi K$ system:

- Employing the $S U(2)$ isospin flavour symmetry of strong interactions, we have given a parametrization of the $B \rightarrow \pi K$ amplitudes, which allows us to deal with $\mathrm{CP}$-violating NP effects in the EW penguin sector. Moreover, the relevant hadronic parameters are introduced in such a manner that we may determine them with the help of the $B \rightarrow \pi \pi$ analysis performed in Section 3. To this end, we have to neglect penguin annihilation and exchange topologies, and have to employ the $S U(3)$ flavour symmetry of strong interactions.

- We find a remarkable agreement between the corresponding determination of $r_{\mathrm{c}}$ and the value following from an alternative strategy, i.e. we arrive at a consistent picture, which would be spoiled if the working assumptions specified in the previous item were not satisfied. On the other hand, if we impose the constraint that the two values of $r_{\mathrm{c}}$ agree with each other, we may refine the extraction of $\gamma$ discussed in Subsection 3.4. In particular, this additional input allows us to resolve the twofold ambiguity, leaving us with $\gamma=\left(64.7_{-6.9}^{+6.3}\right)^{\circ}$, which is in excellent agreement with the SM expectation given in (3.31).

- Having all relevant parameters at hand, we may analyse the $B \rightarrow \pi K$ observables within the SM. As far as the $B_{d} \rightarrow \pi^{\mp} K^{ \pm}, B^{ \pm} \rightarrow \pi^{ \pm} K$ system is concerned, where EW penguins may only enter in colour-suppressed form and are hence expected to play a minor rôle, we arrive at a picture that is in nice agreement with the $B$-factory data and does not indicate any anomalous behaviour. On the other hand, the SM analysis of the observables $R_{\mathrm{c}}$ and $R_{\mathrm{n}}$ of the charged and neutral 
$B \rightarrow \pi K$ systems, respectively, which are significantly affected by EW penguins, is in conflict with the pattern of the $B$-factory data.

- Interestingly, we can resolve this "puzzle" with the help of NP in the EW penguin sector. In particular, we arrive at the values of $q=1.75_{-0.99}^{+1.27}$ and $\phi=-\left(85_{-14}^{+11}\right)^{\circ}$, i.e. at enhanced EW penguins with a large CP-violating NP phase. Moreover, we find the strong phase $\omega=-\left(4_{-32}^{+44}\right)^{\circ}$, in agreement with the implication of the $S U(3)$ flavour symmetry that this phase vanishes. Let us emphasize that this picture corresponds to small direct $\mathrm{CP}$ violation in $B^{ \pm} \rightarrow \pi^{0} K^{ \pm}$, as indicated by the $B$-factory data. Finally, we may predict the CP-violating observables of the $B_{d} \rightarrow \pi^{0} K_{\mathrm{S}}$ channel, which will provide a crucial test of our NP scenario.

- The impact of colour-suppressed EW penguins on our analysis has been addressed in Appendix D. The current $B$-factory data do not indicate any enhancement of these topologies.

- The decays $B_{s} \rightarrow K^{+} K^{-}$and $B_{s} \rightarrow \pi^{ \pm} K^{\mp}$, which are very accessible at hadronic $B$-decay experiments, complement the analysis of the $B_{u, d} \rightarrow \pi \pi, \pi K$ modes proposed above in a variety of ways. In particular the latter channel allows us to avoid assumptions about penguin annihilation and exchange topologies.

\section{$5 \quad$ Rare $K$ and $B$ Decays and $\varepsilon^{\prime} / \varepsilon$}

\subsection{Preliminaries}

As discussed in Section 2, the rare $K$ and $B$ decays are governed by the functions $X, Y$ and $Z$, for which the parametrization in terms of $|C|$ and $\theta_{C}$ was given in (2.8). In order to obtain elegant expressions for rare decays, it is useful to go one step further and to rewrite (2.8) as follows:

$$
X=|X| e^{i \theta_{X}}, \quad Y=|Y| e^{i \theta_{Y}}, \quad Z=|Z| e^{i \theta_{Z}},
$$

with

$$
\begin{aligned}
& |X|=\sqrt{5.52 \bar{q}^{2}-0.42 \bar{q} \cos \phi+0.01}, \quad \tan \theta_{X}=\frac{2.35 \bar{q} \sin \phi}{2.35 \bar{q} \cos \phi-0.09} \\
& |Y|=\sqrt{5.52 \bar{q}^{2}-3.00 \bar{q} \cos \phi+0.41}, \quad \tan \theta_{Y}=\frac{2.35 \bar{q} \sin \phi}{2.35 \bar{q} \cos \phi-0.64} \\
& |Z|=\sqrt{5.52 \bar{q}^{2}-4.42 \bar{q} \cos \phi+0.88}, \quad \tan \theta_{Z}=\frac{2.35 \bar{q} \sin \phi}{2.35 \bar{q} \cos \phi-0.94}
\end{aligned}
$$

It should be emphasized that only two independent parameters, $\bar{q}$ and $\phi$, appear in these expressions.

Next we define the following weak phases:

$$
\beta_{X} \equiv \beta-\beta_{s}-\theta_{X}, \quad \beta_{Y} \equiv \beta-\beta_{s}-\theta_{Y}, \quad \beta_{Z} \equiv \beta-\beta_{s}-\theta_{Z}
$$


In [12], we have suppressed $\beta_{s}$ in all formulae for rare decays but we have included its effect in the numerical analysis.

Finally, the numerical constants in the formulae below correspond to [89] and

$$
\sin ^{2} \theta_{\mathrm{w}}=0.231, \quad \alpha=\frac{1}{128}, \quad \lambda=0.224,
$$

with the first two given in the $\overline{\mathrm{MS}}$ scheme. They are the same as in [39]. The values of the remaining CKM parameters have been given in Section 2.

With the result for $(q, \phi)$ in (4.47) at hand, we can calculate the functions $X, Y$ and $Z$ and the weak phases $\beta_{X}, \beta_{Y}$ and $\beta_{Z}$. Setting $\left|V_{u b} / V_{c b}\right|=0.086$, that is $\bar{q}=q$, we find

$$
|X| \approx|Y| \approx|Z| \approx 4.3_{-2.4}^{+3.0} .
$$

While the central value of $|X|$ is still compatible with the data on $K \rightarrow \pi \nu \bar{\nu}$ and $B \rightarrow$ $X_{s, d} \nu \bar{\nu}$, the central value of $|Y|$ violates the upper bound $|Y| \leq 2.2$ following from the BaBar and Belle data on $B \rightarrow X_{s} \mu^{+} \mu^{-}$[90], and the upper bound on $\mathrm{BR}\left(K_{\mathrm{L}} \rightarrow \pi^{0} e^{+} e^{-}\right)$ in (5.63) from $\mathrm{KTeV}$ [91]. In addition, $|Z|$ is too large to be consistent with the data on $\varepsilon^{\prime} / \varepsilon$, even if the hadronic uncertainties in this ratio are large.

On the other hand, the $B \rightarrow \pi K$ data seem to signal the possibility of enhanced values of $|X|,|Y|$ and $|Z|$ and of large weak phases $\theta_{i}$. Consequently, we may still encounter significant deviations from the SM predictions for rare decays, while being consistent with all experimental data. In order to illustrate this exciting feature, we consider only the subset of those values of $(q, \phi)$ in (4.47) that satisfy the constraint of $|Y|=2.2$. Using (5.3) and expressing $\bar{q}$ in terms of $|Y|$ and $\phi$, and subsequently varying $\phi$ in the full range given in (4.47), we obtain

$$
\bar{q}=0.92_{-0.05}^{+0.07}, \quad \phi=-\left(85_{-14}^{+11}\right)^{\circ} .
$$

These values are compatible with all data on rare decays and also with the $B \rightarrow \pi K$ data. In particular, we find the values for $R_{\mathrm{c}}, R_{\mathrm{n}}, \mathcal{A}_{\mathrm{CP}}^{\mathrm{dir}}\left(B^{ \pm} \rightarrow \pi^{0} K^{ \pm}\right), \mathcal{A}_{\mathrm{CP}}^{\mathrm{dir}}\left(B_{d} \rightarrow \pi^{0} K_{\mathrm{S}}\right)$, $\mathcal{A}_{\mathrm{CP}}^{\operatorname{mix}}\left(B_{d} \rightarrow \pi^{0} K_{\mathrm{S}}\right), L$ and $b$ given in the third column of Table 2 . To this end we have varied $\phi$ in the range given in (5.8), keeping $|Y|=2.2$. We compare the result of this exercise with the values for the $B \rightarrow \pi K$ observables obtained without the $|Y|=2.2$ constraint and with the data.

Proceeding in the same manner, we also find

$$
\begin{array}{ccc}
|C|=2.24 \pm 0.04, \quad \theta_{C}=-(105 \pm 12)^{\circ} \\
|X|=2.17 \pm 0.12, \quad \theta_{X}=-(86 \pm 12)^{\circ}, & \beta_{X}=(111 \pm 12)^{\circ}, \\
|Y|=2.2 \text { (input), } & \theta_{Y}=-(100 \pm 12)^{\circ}, & \beta_{Y}=(124 \pm 12)^{\circ}, \\
|Z|=2.27 \pm 0.06, & \theta_{Z}=-(108 \pm 12)^{\circ}, & \beta_{Z}=(132 \pm 12)^{\circ},
\end{array}
$$

to be compared with $C=0.79, X=1.53, Y=0.98$ and $Z=0.68$ in the SM for $m_{t}=167 \mathrm{GeV}$. We will now turn to the implications of these results for rare $K$ and $B$ decays. 


\begin{tabular}{|c||c|c|c|}
\hline Quantity & Without RD constraint & With RD constraint & Experiment \\
\hline$R_{\mathrm{c}}$ & $1.17 \pm 0.12$ (input) & $1.00_{-0.08}^{+0.12}$ & $1.17 \pm 0.12$ \\
\hline$R_{\mathrm{n}}$ & $0.76 \pm 0.10$ (input) & $0.82_{-0.11}^{+0.12}$ & $0.76 \pm 0.10$ \\
\hline $\mathcal{A}_{\mathrm{CP}}^{\text {dir }}\left(B^{ \pm} \rightarrow \pi^{0} K^{ \pm}\right)$ & $0.04_{-0.28}^{+0.37}$ & $0.03_{-0.24}^{+0.32}$ & $0.00 \pm 0.07$ \\
\hline $\mathcal{A}_{\mathrm{CP}}^{\text {dir }}\left(B_{d} \rightarrow \pi^{0} K_{\mathrm{S}}\right)$ & $0.05_{-0.29}^{+0.24}$ & $0.08_{-0.22}^{+0.18}$ & $0.40_{-0.30}^{+0.29}$ \\
\hline $\mathcal{A}_{\mathrm{CP}}^{\text {mix }}\left(B_{d} \rightarrow \pi^{0} K_{\mathrm{S}}\right)$ & $-0.99_{-0.01}^{+0.04}$ & $-0.98_{-0.02}^{+0.05}$ & $-0.48_{-0.40}^{+0.48}$ \\
\hline$L$ & $6.02_{-4.67}^{+7.37}$ & $2.67_{-0.30}^{+0.34}$ & $5.9_{-2.7}^{+3.0}$ \\
\hline$b$ & $1.24_{-0.15}^{+0.19}$ & $1.15_{-0.13}^{+0.16}$ & $1.19 \pm 0.16$ \\
\hline
\end{tabular}

Table 2: Theoretical predictions with and without the $|Y|=2.2$ rare decays (RD) constraint and experimental data for the most important observables.

\section{$5.2 \quad K \rightarrow \pi \nu \bar{\nu}$}

\subsubsection{Basic Formulae}

The rare decays $K^{+} \rightarrow \pi^{+} \nu \bar{\nu}$ and $K_{\mathrm{L}} \rightarrow \pi^{0} \nu \bar{\nu}$ proceed through $Z^{0}$-penguin and box diagrams. As the required hadronic matrix elements can be extracted from the leading semileptonic decays and other long-distance contributions turn out to be negligible [92], the relevant branching ratios can be computed to an exceptionally high degree of precision [93]-[95]. In [31]-[33], these decays have already been discussed in the NP scenario considered here. Below we update the formulae of these papers, adapt them to our notation, derive a few new ones, and include $\mathcal{O}\left(\lambda^{2}\right)$ terms that were neglected there. The branching ratios are then given as follows:

$$
\begin{gathered}
\operatorname{BR}\left(K^{+} \rightarrow \pi^{+} \nu \bar{\nu}\right)=4.78 \times 10^{-11} \times\left[\tilde{r}^{2} A^{4} R_{t}^{2}|X|^{2}+2 \tilde{r} \bar{P}_{c}(X) A^{2} R_{t}|X| \cos \beta_{X}+\bar{P}_{c}(X)^{2}\right] \\
\operatorname{BR}\left(K_{\mathrm{L}} \rightarrow \pi^{0} \nu \bar{\nu}\right)=2.09 \times 10^{-10} \times \tilde{r}^{2} A^{4} R_{t}^{2}|X|^{2} \sin ^{2} \beta_{X}
\end{gathered}
$$

with

$$
\bar{P}_{c}(X)=\left(1-\frac{\lambda^{2}}{2}\right) P_{c}(X),
$$

where $P_{c}(X)=0.39 \pm 0.06$ results from the internal charm contribution [93, 94], $\beta_{X}$ is defined in (5.5) and $\tilde{r}$ in (2.16).

Once $\operatorname{BR}\left(K^{+} \rightarrow \pi^{+} \nu \bar{\nu}\right)$ and $\operatorname{BR}\left(K_{\mathrm{L}} \rightarrow \pi^{0} \nu \bar{\nu}\right)$ have been measured, the parameters $|X|$ and $\beta_{X}$ can be determined, subject to ambiguities that can be resolved by considering other processes, such as the non-leptonic $B$ decays discussed before and the rare decays discussed below. Combining (5.13) and (5.14), we find

$$
r_{s}=\frac{\varepsilon_{1} \sqrt{B_{1}-B_{2}}-\bar{P}_{c}(X)}{\varepsilon_{2} \sqrt{B_{2}}}=\cot \beta_{X}
$$

where $\varepsilon_{i}= \pm 1$, and consequently

$$
\sin 2 \beta_{X}=\frac{2 r_{s}}{1+r_{s}^{2}} .
$$


Moreover,

$$
|X|=\frac{\varepsilon_{2} \sqrt{B_{2}}}{\tilde{r} A^{2} R_{t} \sin \beta_{X}}, \quad \varepsilon_{2} \sin \beta_{X}>0 .
$$

The "reduced" branching ratios $B_{i}$ are given by

$$
B_{1}=\frac{\mathrm{BR}\left(K^{+} \rightarrow \pi^{+} \nu \bar{\nu}\right)}{4.78 \times 10^{-11}}, \quad B_{2}=\frac{\mathrm{BR}\left(K_{\mathrm{L}} \rightarrow \pi^{0} \nu \bar{\nu}\right)}{2.09 \times 10^{-10}} .
$$

The formulae (5.17) and (5.18) were already presented in [32]. They are valid for arbitrary $\beta_{X} \neq 0^{\circ}$ and generalize the ones given for the SM and MFV models in [96] and [97], respectively. For $\theta_{X}=0^{\circ}$ and $\varepsilon_{1}=\varepsilon_{2}=1$, one obtains from (5.17) the SM result that differs at first sight from the one given in [96]. However, in that paper and subsequent studies in the literature, a formula for $\sin 2 \beta$ and not $\sin 2\left(\beta-\beta_{s}\right)$ was given. Using the fact that $\beta_{s}=\mathcal{O}\left(\lambda^{2}\right)$, one can verify that (5.17), while being slightly more accurate, is numerically very close to the formula of [96]. In the scenario considered here, we have $99^{\circ} \leq \beta_{X} \leq 125^{\circ}$ and, consequently, $\varepsilon_{1}=-1$ and $\varepsilon_{2}=1$.

It should be stressed that $\sin 2 \beta_{X}$ determined this way depends only on two measurable branching ratios and on $\bar{P}_{c}(X)$, which is completely calculable in perturbation theory. Consequently, this determination is free from any hadronic uncertainties and its accuracy can be estimated with a high degree of confidence. As in our scenario $\beta$ and $\beta_{s}$ are already known from the usual analysis of the UT, the measurement of $r_{s}$ in $K \rightarrow \pi \nu \bar{\nu}$ decays will provide a theoretically clean determination of $\theta_{X}$. Similarly, a clean determination of $|X|$ is possible by means of (5.18), so that (5.2) will allow us to determine $\bar{q}$ and $\phi$. Assuming that the measurements of $\mathrm{BR}\left(K^{+} \rightarrow \pi^{+} \nu \bar{\nu}\right)$ and $\mathrm{BR}\left(K_{\mathrm{L}} \rightarrow \pi^{0} \nu \bar{\nu}\right)$ with $10 \%$ accuracy will be performed one day, the decays in question will most probably provide the cleanest measurements of $\bar{q}$ and $\phi$. This determination could then be compared with the one from other decays, in particular from $\mathcal{A}_{\mathrm{CP}}^{\mathrm{dir}}\left(B_{d} \rightarrow \pi^{0} K_{\mathrm{S}}\right)$ and $\mathcal{A}_{\mathrm{CP}}^{\operatorname{mix}}\left(B_{d} \rightarrow \pi^{0} K_{\mathrm{S}}\right)$ that we proposed in Section 4 . It could also be used to calculate other $B \rightarrow \pi K$ observables.

\subsubsection{Numerical Results}

Using the results for $\bar{q}$ and $\phi$ in (5.8) and the parameters in (2.19), (2.20) and (5.6), we find

$$
\mathrm{BR}\left(K^{+} \rightarrow \pi^{+} \nu \bar{\nu}\right)=(7.5 \pm 2.1) \times 10^{-11}, \quad \mathrm{BR}\left(K_{\mathrm{L}} \rightarrow \pi^{0} \nu \bar{\nu}\right)=(3.1 \pm 1.0) \times 10^{-10}
$$

This should be compared with the SM prediction, for which we find

$$
\mathrm{BR}\left(K^{+} \rightarrow \pi^{+} \nu \bar{\nu}\right)_{\mathrm{SM}}=(8.0 \pm 1.1) \times 10^{-11}, \quad \mathrm{BR}\left(K_{\mathrm{L}} \rightarrow \pi^{0} \nu \bar{\nu}\right)_{\mathrm{SM}}=(3.2 \pm 0.6) \times 10^{-11}
$$

in the ballpark of other recent estimates [92, 98]. On the experimental side, the results of the AGS E787 [99] and KTeV [100] collaborations are

$$
\mathrm{BR}\left(K^{+} \rightarrow \pi^{+} \nu \bar{\nu}\right)=\left(15.7_{-8.2}^{+17.5}\right) \times 10^{-11} \text { and } \operatorname{BR}\left(K_{\mathrm{L}} \rightarrow \pi^{0} \nu \bar{\nu}\right)<5.9 \times 10^{-7} \text {, }
$$

respectively. 
We observe that in our scenario $\operatorname{BR}\left(K^{+} \rightarrow \pi^{+} \nu \bar{\nu}\right)$ does not significantly differ from the SM estimate because the enhancement of the first term in (5.13) is to a large extent compensated by the suppression of the second term $\left(\cos \beta_{X} \ll \cos \left(\beta-\beta_{s}\right)\right)$ and its reversed sign. Consequently, $\mathrm{BR}\left(K^{+} \rightarrow \pi^{+} \nu \bar{\nu}\right)$ is very strongly dominated by the "top" contribution given by the function $X$.

On the other hand, we observe a spectacular enhancement of $\mathrm{BR}\left(K_{\mathrm{L}} \rightarrow \pi^{0} \nu \bar{\nu}\right)$ by one order of magnitude. Consequently, while $\mathrm{BR}\left(K_{\mathrm{L}} \rightarrow \pi^{0} \nu \bar{\nu}\right) \approx(1 / 3) \mathrm{BR}\left(K^{+} \rightarrow \pi^{+} \nu \bar{\nu}\right)$ in the $\mathrm{SM}$, it is substantially larger than $\operatorname{BR}\left(K^{+} \rightarrow \pi^{+} \nu \bar{\nu}\right)$ in our scenario. The huge enhancement of $\mathrm{BR}\left(K_{\mathrm{L}} \rightarrow \pi^{0} \nu \bar{\nu}\right)$ found here is mainly due to the large weak phase $\beta_{X} \approx 111^{\circ}$, as

$$
\frac{\mathrm{BR}\left(K_{\mathrm{L}} \rightarrow \pi^{0} \nu \bar{\nu}\right)}{\operatorname{BR}\left(K_{\mathrm{L}} \rightarrow \pi^{0} \nu \bar{\nu}\right)_{\mathrm{SM}}}=\left|\frac{X}{X_{\mathrm{SM}}}\right|^{2}\left[\frac{\sin \beta_{X}}{\sin \left(\beta-\beta_{s}\right)}\right]^{2} .
$$

Inspecting (5.13) and (5.14), we observe that the very strong dominance of the "top" contribution in these expressions implies a simple approximate expression:

$$
\frac{\operatorname{BR}\left(K_{\mathrm{L}} \rightarrow \pi^{0} \nu \bar{\nu}\right)}{\operatorname{BR}\left(K^{+} \rightarrow \pi^{+} \nu \bar{\nu}\right)} \approx 4.4 \times\left(\sin \beta_{X}\right)^{2} \approx 4.2 \pm 0.2 .
$$

We note that $\mathrm{BR}\left(K_{\mathrm{L}} \rightarrow \pi^{0} \nu \bar{\nu}\right)$ is then rather close to its model-independent upper bound [101]

$$
\mathrm{BR}\left(K_{\mathrm{L}} \rightarrow \pi^{0} \nu \bar{\nu}\right) \leq 4.4 \mathrm{BR}\left(K^{+} \rightarrow \pi^{+} \nu \bar{\nu}\right) .
$$

It is evident from (5.16) that this bound is reached when the reduced branching ratios $B_{1}$ and $B_{2}$ in (5.19) are equal to each other.

A spectacular implication of these findings is a strong violation of the relation [96]

$$
(\sin 2 \beta)_{\pi \nu \bar{\nu}}=(\sin 2 \beta)_{\psi K_{\mathrm{S}}},
$$

which is valid in the SM and any model with MFV. Indeed, we find

$$
(\sin 2 \beta)_{\pi \nu \bar{\nu}}=\sin 2 \beta_{X}=-\left(0.69_{-0.41}^{+0.23}\right),
$$

in striking disagreement with $(\sin 2 \beta)_{\psi K_{\mathrm{S}}}=0.736 \pm 0.049$ following from (1.8).

In Fig. 11, we plot - in the spirit of [97] $-\mathrm{BR}\left(K^{+} \rightarrow \pi^{+} \nu \bar{\nu}\right)$ as a function of $\mathrm{BR}\left(K_{\mathrm{L}} \rightarrow\right.$ $\left.\pi^{0} \nu \bar{\nu}\right)$ for fixed values of $\beta_{X}$. As this plot is independent of $|X|$, it offers a direct measurement of the phase $\beta_{X}$. The first line on the left represents the MFV models with $\beta_{X}=\beta-\beta_{s}$, whereas the first line on the right corresponds to the model-independent Grossman-Nir bound [101] given in (5.25). The central value $\beta_{X}=111^{\circ}$ found here is very close to this bound. Note that the value of $\beta_{X}$ corresponding to this bound, where $B_{1}=B_{2}$, depends on the actual value of these reduced branching ratios. As can be easily seen from (5.16), we have

$$
\left(\cot \beta_{X}\right)_{\text {Bound }}=-\frac{\bar{P}_{c}(X)}{\varepsilon_{2} \sqrt{B_{2}}} .
$$

For the central values of $\bar{P}_{c}(X)$ and $B_{2}$ found here the bound corresponds to $\beta_{X}=107.3^{\circ}$. As only $\cot \beta_{X}$ and not $\beta_{X}$ is directly determined by the values of the branching ratios in question, the angle $\beta_{X}$ is determined only up to discrete ambiguities, seen already in Fig. 11. These ambiguities can be resolved by considering simultaneously other quantities discussed in our paper. The corresponding plot for different values of $\beta_{X}$ that are close to $\beta$ can be found in [97]. 


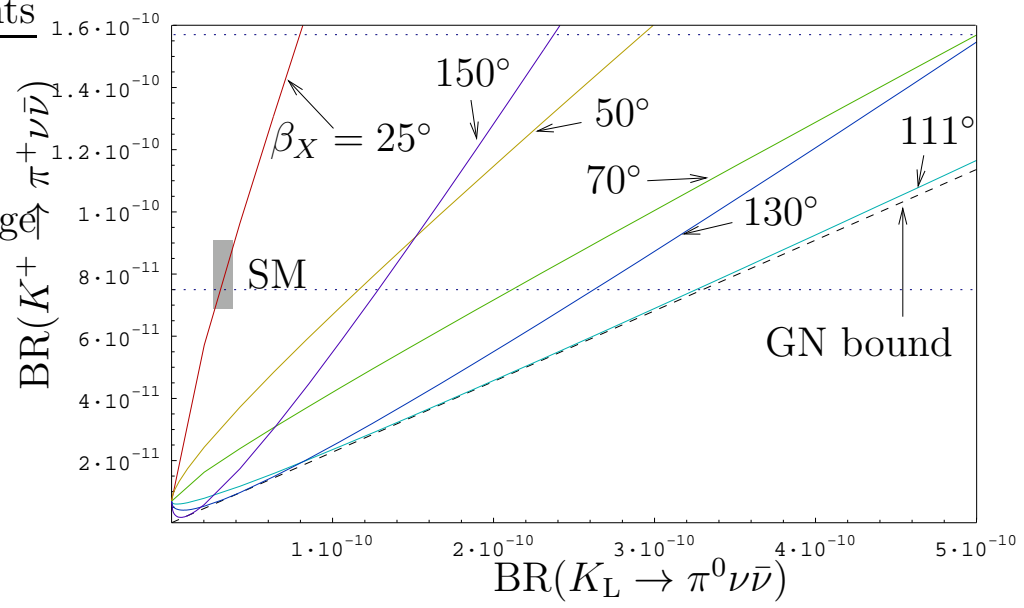

Figure 11: $\mathrm{BR}\left(K^{+} \rightarrow \pi^{+} \nu \bar{\nu}\right)$ as a function of $\mathrm{BR}\left(K_{\mathrm{L}} \rightarrow \pi^{0} \nu \bar{\nu}\right)$ for various values of $\beta_{X}$. The dotted horizontal lines indicate the lower part of the experimental range (5.22) and the grey area the SM prediction. We also show the bound in (5.25).

\section{$5.3 \quad B \rightarrow X_{s, d} \nu \bar{\nu}$ and $B_{s, d} \rightarrow \mu^{+} \mu^{-}$}

The inclusive decays $B \rightarrow X_{s, d} \nu \bar{\nu}$ are also theoretically clean [93, 102]. Generalizing the known MFV formula to our NP scenario, we obtain

$$
\mathrm{BR}\left(B \rightarrow X_{q} \nu \bar{\nu}\right)=1.58 \times 10^{-5}\left[\frac{\mathrm{BR}\left(B \rightarrow X_{c} e \bar{\nu}\right)}{0.104}\right]\left[\frac{0.54}{f(z)}\right] \frac{\left|V_{t q}\right|^{2}}{\left|V_{c b}\right|^{2}}|X(v)|^{2},
$$

where $q=s, d$, and $f(z)$ is a phase-space factor for $B \rightarrow X_{c} e \bar{\nu}$. The SM expectation

$$
\mathrm{BR}\left(B \rightarrow X_{s} \nu \bar{\nu}\right)_{\mathrm{SM}}=(3.5 \pm 0.5) \times 10^{-5}
$$

is to be compared with the $90 \%$ C.L. ALEPH upper bound of $6.4 \times 10^{-4}$. The exclusive channels are less clean but experimentally more easily accessible, with the $90 \%$ C.L. BaBar upper bound of $7.0 \times 10^{-5}$.

Next, the branching ratios for the rare decays $B_{q} \rightarrow \mu^{+} \mu^{-}$are given by

$$
\begin{gathered}
\operatorname{BR}\left(B_{s} \rightarrow \mu^{+} \mu^{-}\right)=2.42 \times 10^{-6} \times\left[\frac{\tau_{B_{s}}}{1.46 \mathrm{ps}}\right]\left[\frac{F_{B_{s}}}{238 \mathrm{MeV}}\right]^{2}\left|V_{t b}^{*} V_{t s}\right|^{2}|Y(v)|^{2} \\
\operatorname{BR}\left(B_{d} \rightarrow \mu^{+} \mu^{-}\right)=1.82 \times 10^{-6} \times\left[\frac{\tau_{B_{d}}}{1.54 \mathrm{ps}}\right]\left[\frac{F_{B_{d}}}{203 \mathrm{MeV}}\right]^{2}\left|V_{t b}^{*} V_{t d}\right|^{2}|Y(v)|^{2},
\end{gathered}
$$

with $\tau_{B_{d, s}}$ and $F_{B_{d, s}}$ being the lifetime and decay constants, respectively.

In the SM, we find [103]

$$
\begin{gathered}
\mathrm{BR}\left(B_{s} \rightarrow \mu^{+} \mu^{-}\right)_{\mathrm{SM}}=(3.42 \pm 0.53)\left[\frac{\Delta M_{s}}{18.0 / \mathrm{ps}}\right] \times 10^{-9} \\
\mathrm{BR}\left(B_{d} \rightarrow \mu^{+} \mu^{-}\right)_{\mathrm{SM}}=(1.00 \pm 0.14) \times 10^{-10}
\end{gathered}
$$


This should be compared with the $90 \%$ C.L. bounds

$$
\operatorname{BR}\left(B_{s} \rightarrow \mu^{+} \mu^{-}\right)<9.5(16) \times 10^{-7} \text { and } \operatorname{BR}\left(B_{d} \rightarrow \mu^{+} \mu^{-}\right)<1.6 \times 10^{-7}
$$

from the CDF (D0) and Belle collaborations, respectively [104, 105].

Inspecting formulae (5.2) and (5.3), we observe that for a fixed value of $\bar{q}$, the effect of a non-vanishing phase $\phi \neq 0^{\circ}$ is to suppress $|X|$ and $|Y|$. Thus, in spite of an enhancement of $\bar{q}$, it is possible that for certain values of $\phi$ a suppression of the branching ratios given in (5.29), (5.31) and (5.32) relative to the SM expectations could be found. However, this does not happen in our case, and we find

$$
\frac{\mathrm{BR}\left(B \rightarrow X_{s} \nu \bar{\nu}\right)}{\operatorname{BR}\left(B \rightarrow X_{s} \nu \bar{\nu}\right)_{\mathrm{SM}}}=\left|\frac{X}{X_{\mathrm{SM}}}\right|^{2} \approx 2.0 \quad \text { and } \quad \frac{\mathrm{BR}\left(B_{s} \rightarrow \mu^{+} \mu^{-}\right)}{\mathrm{BR}\left(B_{s} \rightarrow \mu^{+} \mu^{-}\right)_{\mathrm{SM}}}=\left|\frac{Y}{Y_{\mathrm{SM}}}\right|^{2} \approx 5.0,
$$

with the same enhancements for $B \rightarrow X_{d} \nu \bar{\nu}$ and $B_{d} \rightarrow \mu^{+} \mu^{-}$, respectively. We also find $\operatorname{BR}\left(B \rightarrow X_{s} \nu \bar{\nu}\right) \approx 7 \times 10^{-5}, \operatorname{BR}\left(B_{s} \rightarrow \mu^{+} \mu^{-}\right) \approx 17 \times 10^{-9}, \operatorname{BR}\left(B_{d} \rightarrow \mu^{+} \mu^{-}\right) \approx 5 \times 10^{-10}$, which are still well below the experimental bounds.

\section{$5.4 \quad K_{\mathrm{L}} \rightarrow \mu^{+} \mu^{-}$}

For the short-distance contribution to the dispersive part of $K_{\mathrm{L}} \rightarrow \mu^{+} \mu^{-}$, we obtain in our NP scenario

$$
\operatorname{BR}\left(K_{\mathrm{L}} \rightarrow \mu^{+} \mu^{-}\right)_{\mathrm{SD}}=1.95 \times 10^{-9} \times\left[\bar{P}_{c}(Y)+A^{2} R_{t}|Y(v)| \cos \beta_{Y}\right]^{2},
$$

where $\beta_{Y}$ is defined in (5.5), and

$$
\bar{P}_{c}(Y)=\left(1-\frac{\lambda^{2}}{2}\right) P_{c}(Y)
$$

with $P_{c}(Y)=0.121 \pm 0.012$ [94]. Unfortunately, because of long-distance contributions to the dispersive part of $K_{\mathrm{L}} \rightarrow \mu^{+} \mu^{-}$, the extraction of $\mathrm{BR}\left(K_{\mathrm{L}} \rightarrow \mu^{+} \mu^{-}\right)_{\mathrm{SD}}$ from the data is subject to considerable uncertainties [106, 107]. While the chapter on this extraction is certainly not closed, let us quote the estimate of [106], which reads

$$
\mathrm{BR}\left(K_{\mathrm{L}} \rightarrow \mu^{+} \mu^{-}\right)_{\mathrm{SD}} \leq 2.5 \times 10^{-9},
$$

to be compared with $\mathrm{BR}\left(K_{\mathrm{L}} \rightarrow \mu^{+} \mu^{-}\right)_{\mathrm{SD}}^{\mathrm{SM}}=(0.8 \pm 0.3) \times 10^{-9}$ in the SM.

In the scenario with enhanced $Z^{0}$ penguins but no new weak phases considered by us in [16], $\mathrm{BR}\left(K_{\mathrm{L}} \rightarrow \mu^{+} \mu^{-}\right)_{\mathrm{SD}}$ was substantially enhanced; in the present case, however, with $\theta_{Y} \neq 0^{\circ}$, the small value of $\cos \beta_{Y}$ compensates the enhancement of $|Y|$, so that we find

$$
\operatorname{BR}\left(K_{\mathrm{L}} \rightarrow \mu^{+} \mu^{-}\right)_{\mathrm{SD}}=(0.9 \pm 0.6) \times 10^{-9} .
$$




\subsection{Forward-Backward Asymmetries in $b \rightarrow s \mu^{+} \mu^{-}$Modes}

\subsubsection{Basic Formulae}

It has been pointed out in [34] that the exclusive decays $B_{d} \rightarrow K^{*} \mu^{+} \mu^{-}$and their inclusive counterparts $B \rightarrow X_{s} \mu^{+} \mu^{-}$offer excellent means to probe enhanced $Z^{0}$ penguins and, in particular, their complex weak phases.

During the last two years significant progress for these transitions has been made both by experimentalists and theorists. On the experimental side, the Belle and BaBar collaborations [90] reported the observation of this decay and of the $B \rightarrow X_{s} e^{+} e^{-}$channel. The $90 \%$ C.L. ranges extracted from these papers [108] read as follows:

$$
\begin{aligned}
& 3.5 \times 10^{-6} \leq \mathrm{BR}\left(B \rightarrow X_{s} \mu^{+} \mu^{-}\right) \leq 10.4 \times 10^{-6} \\
& 2.8 \times 10^{-6} \leq \mathrm{BR}\left(B \rightarrow X_{s} e^{+} e^{-}\right) \leq 8.8 \times 10^{-6} .
\end{aligned}
$$

On the theoretical side, important NNLO corrections have been calculated in [109]-[111]. The most recent reviews summarizing the theoretical status can be found in $[112,113]$. The NNLO formulae are very complicated and it is not the purpose of our paper to present a detailed NNLO analysis here. For this reason, we give below NLO formulae that show transparently the size of various effects that we could expect in our scenario.

Let us recall that these decays are dominated by the operators

$$
Q_{9 V}=(\bar{s} b)_{V-A}(\bar{\mu} \mu)_{V}, \quad Q_{10 A}=(\bar{s} b)_{V-A}(\bar{\mu} \mu)_{A},
$$

which are generated through EW penguin diagrams. At low

$$
\hat{s}=\frac{\left(p_{\mu^{+}}+p_{\mu^{-}}\right)^{2}}{m_{b}^{2}},
$$

also the magnetic operator $Q_{7 \gamma}$ plays a significant rôle.

At the NLO level $[114,115]$, the invariant dilepton mass spectrum in the inclusive decay $B \rightarrow X_{s} \mu^{+} \mu^{-}$is given by

$$
\frac{d / d \hat{s} \Gamma\left(b \rightarrow s \mu^{+} \mu^{-}\right)}{\Gamma(b \rightarrow c e \bar{\nu})}=\frac{\alpha^{2}}{4 \pi^{2}}\left|\frac{V_{t s}}{V_{c b}}\right|^{2} \frac{(1-\hat{s})^{2}}{f(z) \kappa(z)} U(\hat{s}),
$$

where

$$
U(\hat{s})=(1+2 \hat{s})\left(\left|\tilde{C}_{9}^{\text {eff }}(\hat{s})\right|^{2}+\left|\tilde{C}_{10}\right|^{2}\right)+4\left(1+\frac{2}{\hat{s}}\right)\left|C_{7 \gamma}^{(0) \text { eff }}\right|^{2}+12 C_{7 \gamma}^{(0) \text { eff }} \operatorname{Re} \tilde{C}_{9}^{\text {eff }}(\hat{s})
$$

and $\tilde{C}_{9}^{\text {eff }}(\hat{s})$ is a function of $\hat{s}$ that depends on the Wilson coefficient $\tilde{C}_{9}$ and includes also contributions from four-quark operators. $C_{7 \gamma}^{(0) \text { eff }}$ is the Wilson coefficient of the magnetic operator $Q_{7 \gamma}$. Explicit formulae can be found in $[114,115]$. The Wilson coefficients $\tilde{C}_{9}$ and $\tilde{C}_{10}$ are given as follows:

$$
\tilde{C}_{9}(v)=P_{0}+\frac{Y(v)}{\sin ^{2} \theta_{\mathrm{w}}}-4 Z(v)+P_{E} E(v), \quad \tilde{C}_{10}(v)=-\frac{Y(v)}{\sin ^{2} \theta_{\mathrm{w}}},
$$


with $P_{0}=2.60 \pm 0.25$ in the NDR scheme, $P_{E}=\mathcal{O}\left(10^{-2}\right)$ and $v$ denoting collectively the parameters involved. $\tilde{C}_{9}$ and $\tilde{C}_{10}$ are defined by

$$
C_{9 V}(v)=\frac{\alpha}{2 \pi} \tilde{C}_{9}(v), \quad C_{10 A}(v)=\frac{\alpha}{2 \pi} \tilde{C}_{10}(v) .
$$

Of particular interest is the forward-backward asymmetry in $B \rightarrow X_{s} \mu^{+} \mu^{-}$[116]. It becomes non-zero only at the NLO level. In our scenario it is given - in this approximation - as follows:

$$
A_{\mathrm{FB}}(\hat{s})=-3 \operatorname{Re}\left[\tilde{C}_{10}^{*} \frac{\hat{s} \tilde{C}_{9}^{\mathrm{eff}}(\hat{s})+2 C_{7 \gamma}^{(0) \text { eff }}}{U(\hat{s})}\right],
$$

where $U(\hat{s})$ is given in (5.47). The expression for the corresponding asymmetry in the exclusive decay $B_{d} \rightarrow K^{*} \mu^{+} \mu^{-}$can be found in [34]. Both asymmetries vanish at a certain $\hat{s}=\hat{s}_{0}$ [117], which is determined in the case of the inclusive decay considered here through

$$
\hat{s}_{0} \operatorname{Re} \tilde{C}_{9}^{\text {eff }}\left(\hat{s}_{0}\right)+2 C_{7 \gamma}^{(0) \text { eff }}=0 .
$$

As in our scenario $\tilde{C}_{9}^{\text {eff }}\left(\hat{s}_{0}\right)$ and $C_{7 \gamma}^{(0) \text { eff }}$ are assumed to be marginally affected by NP contributions, the value of $\hat{s}_{0}$ is bound to be very close to the one predicted in the SM $[109,110]: \hat{s}_{0}=0.162 \pm 0.008$ after the inclusion of NNLO corrections. On the other hand, as we will see below, the magnitude of $A_{\mathrm{FB}}(\hat{s})$ and its sign could be strongly affected by the size of $\tilde{C}_{10}$ and its phase $\theta_{Y}$.

Equally interesting is the forward-backward CP asymmetry introduced for $B_{d} \rightarrow$ $K^{*} \mu^{+} \mu^{-}$in [34], with the explicit expression given in equation (63) of that paper. This asymmetry can be substantially different from zero above the $c \bar{c}$ threshold if $\widetilde{C}_{10}$ contains a large weak phase. The integrated asymmetry is found then to be [34]

$$
\Delta A_{\mathrm{FB}}^{\mathrm{CP}}=\int_{0.52}^{0.69} d \hat{s} A_{\mathrm{FB}}^{\mathrm{CP}}(\hat{s})=(0.03 \pm 0.01) \times \frac{\operatorname{Im} \tilde{C}_{10}}{\operatorname{Re} \tilde{C}_{10}}
$$

where the range of the integration is chosen to decrease the effect of non-perturbative uncertainties.

\subsubsection{Numerical Results}

Using the NNLO formulae of [113] and comparing with the Belle and BaBar data for the branching ratios in (5.42) and (5.43) allows us to obtain a conservative upper bound

$$
|Y| \leq 2.2
$$

which we already used in our previous numerical analysis [16]. Taking this upper bound into account and the values of $|Y|$ and $\theta_{Y}$ in (5.11), we find the following results that were anticipated in [34] in the case of a large CP-violating weak phase in $\tilde{C}_{10}$. Our scenario is a concrete realization of such a situation. The most important findings are the following: 
- As

$$
\operatorname{sgn}\left(\operatorname{Re} \tilde{C}_{10}\right)=-\operatorname{sgn}(\operatorname{Re} Y)=-\operatorname{sgn}\left(\cos \theta_{Y}\right)
$$

governs the sign of $A_{\mathrm{FB}}(\hat{s})$, and $\theta_{Y}=0^{\circ}$ in the $\mathrm{SM}$, in our scenario with $\theta_{Y}>90^{\circ}$, the sign of $A_{\mathrm{FB}}(\hat{s})$ is opposite to the one in the SM: the asymmetry is positive for $\hat{s}<\hat{s}_{0}$ and negative for $\hat{s}>\hat{s}_{0}$. The value of $\hat{s}_{0}$ is essentially unaffected.

- However,

$$
\frac{\operatorname{Re} \tilde{C}_{10}}{\left(\operatorname{Re} \tilde{C}_{10}\right)_{\mathrm{SM}}}=\frac{|Y|}{|Y|_{\mathrm{SM}}} \cos \theta_{Y} \approx-0.5,
$$

which through (5.50) implies the suppression of $A_{\mathrm{FB}}(\hat{s})$ in our scenario with respect to the SM case.

- On the other hand,

$$
\Delta A_{\mathrm{FB}}^{\mathrm{CP}}=(0.03 \pm 0.01) \times \tan \theta_{Y}= \begin{cases}-0.06 \pm 0.02 & \left(\theta_{Y}=115^{\circ}\right) \\ -0.11 \pm 0.04 & \left(\theta_{Y}=105^{\circ}\right) \\ -0.34 \pm 0.11 & \left(\theta_{Y}=95^{\circ}\right)\end{cases}
$$

is still another spectacular effect of the large weak phase $\phi$ implied by the $B \rightarrow \pi K$ data. The large sensitivity of $\Delta A_{\mathrm{FB}}^{\mathrm{CP}}$ to $\theta_{Y}$ for $\theta_{Y}=\mathcal{O}\left(100^{\circ}\right)$ offers a useful method for the determination of this phase.

\section{$5.6 \quad K_{\mathrm{L}} \rightarrow \pi^{0} e^{+} e^{-}$}

The rare decay $K_{\mathrm{L}} \rightarrow \pi^{0} e^{+} e^{-}$is dominated by CP-violating contributions. It has recently been reconsidered within the SM [118] in view of the most recent NA48 data on $K_{\mathrm{S}} \rightarrow \pi^{0} e^{+} e^{-}$and $K_{\mathrm{L}} \rightarrow \pi^{0} \gamma \gamma$ [119], which allow a much better evaluation of the $\mathrm{CP}$-conserving and indirectly (mixing) CP-violating contributions. The CP-conserving part is found to be below $3 \times 10^{-12}$. Moreover, in the SM the indirectly (mixing) CPviolating contribution and the interference of both $\mathrm{CP}$-violating contributions dominate the branching ratio in question, while the directly $\mathrm{CP}$-violating contribution alone is significantly smaller and in the ballpark of $4 \times 10^{-12}$. In our scenario, this pattern is significantly changed, the latter part becoming the dominant contribution. Indeed, similar to $\mathrm{BR}\left(K_{\mathrm{L}} \rightarrow \pi^{0} \nu \bar{\nu}\right)$, the directly CP-violating contribution to $\mathrm{BR}\left(K_{\mathrm{L}} \rightarrow \pi^{0} e^{+} e^{-}\right)$is enhanced by more than one order of magnitude.

Generalizing formula (33) in [118] to our scenario, we find

$$
\mathrm{BR}\left(K_{\mathrm{L}} \rightarrow \pi^{0} e^{+} e^{-}\right)_{\mathrm{CPV}}=10^{-12} \times\left[C_{\mathrm{mix}}+\bar{C}_{\mathrm{int}}\left(\frac{\kappa}{3 \times 10^{-4}}\right)+\bar{C}_{\mathrm{dir}}\left(\frac{\kappa}{3 \times 10^{-4}}\right)^{2}\right],
$$

where

$$
\begin{gathered}
\kappa=\frac{\operatorname{Im} \lambda_{t}}{\sin \left(\beta-\beta_{s}\right)}=\tilde{r} A^{2} \lambda^{5} R_{t}, \quad \tilde{r}=\left|\frac{V_{t s}}{V_{c b}}\right| \approx 0.98, \\
C_{\text {mix }}=(15.7 \pm 0.3)\left|a_{s}\right|^{2}, \quad\left|a_{s}\right|=1.08_{-0.21}^{+0.26}, \\
\bar{C}_{\text {int }}=1.02 \hat{y}_{7 V} \sqrt{C_{\text {mix }}}, \quad \bar{C}_{\text {dir }}=0.56\left(\hat{y}_{7 A}^{2}+\hat{y}_{7 V}^{2}\right) .
\end{gathered}
$$


Here

$$
\begin{gathered}
\hat{y}_{7 V}=\left[P_{0}+P_{E} E(v)\right] \sin \left(\beta-\beta_{s}\right)+\frac{|Y(v)|}{\sin ^{2} \theta_{\mathrm{w}}} \sin \beta_{Y}-4|Z(v)| \sin \beta_{Z} \\
\hat{y}_{7 A}=-\frac{|Y(v)|}{\sin ^{2} \theta_{\mathrm{w}}} \sin \beta_{Y},
\end{gathered}
$$

where $P_{0}=2.89 \pm 0.06[120]$ and $P_{E}$ is $\mathcal{O}\left(10^{-2}\right)$. The effect of the NP contributions is mainly felt in $\hat{y}_{7 A}$, as the corresponding contributions in $\hat{y}_{7 V}$ cancel each other to a large extent.

The present experimental bound from $\mathrm{KTeV}$ [91],

$$
\operatorname{BR}\left(K_{\mathrm{L}} \rightarrow \pi^{0} e^{+} e^{-}\right)<2.8 \times 10^{-10} \quad(90 \% \text { C.L. }),
$$

should be compared with the SM prediction [118],

$$
\mathrm{BR}\left(K_{\mathrm{L}} \rightarrow \pi^{0} e^{+} e^{-}\right)_{\mathrm{SM}}=\left(3.2_{-0.8}^{+1.2}\right) \times 10^{-11}
$$

The enhancement of $\sin \beta_{Y}$ over $\sin \left(\beta-\beta_{s}\right)$ and of $|Y(v)|$ over $Y$ makes the directly $\mathrm{CP}$-violating contribution the dominant part of the branching ratio, for which we find

$$
\mathrm{BR}\left(K_{\mathrm{L}} \rightarrow \pi^{0} e^{+} e^{-}\right)_{\mathrm{CPV}}=(7.8 \pm 1.6) \times 10^{-11},
$$

which is lower than the upper bound in (5.63) by only a factor of 3 .

\section{$5.7 \varepsilon^{\prime} / \varepsilon$}

The formula for the CP-violating ratio $\varepsilon^{\prime} / \varepsilon$ of [121] generalizes for the NP scenario considered here as follows:

$$
\frac{\varepsilon^{\prime}}{\varepsilon}=\tilde{r} A^{2} R_{t} \lambda^{5} \times \tilde{F}_{\varepsilon^{\prime}}(v)
$$

with

$\tilde{F}_{\varepsilon^{\prime}}(v)=\left[P_{0}+P_{E} E(v)\right] \sin \left(\beta-\beta_{s}\right)+P_{X}|X(v)| \sin \beta_{X}+P_{Y}|Y(v)| \sin \beta_{Y}+P_{Z}|Z(v)| \sin \beta_{Z}$.

Here the $P_{i}$ encode the information about the physics at scales $\mu \leq \mathcal{O}\left(m_{t}, M_{W}\right)$, and are given in terms of the hadronic parameters

$$
R_{6} \equiv B_{6}^{(1 / 2)}\left[\frac{121 \mathrm{MeV}}{m_{s}\left(m_{c}\right)+m_{d}\left(m_{c}\right)}\right]^{2}, \quad R_{8} \equiv B_{8}^{(3 / 2)}\left[\frac{121 \mathrm{MeV}}{m_{s}\left(m_{c}\right)+m_{d}\left(m_{c}\right)}\right]^{2}
$$

as follows:

$$
P_{i}=r_{i}^{(0)}+r_{i}^{(6)} R_{6}+r_{i}^{(8)} R_{8}
$$

The coefficients $r_{i}^{(0)}, r_{i}^{(6)}$ and $r_{i}^{(8)}$ comprise information on the Wilson-coefficient functions of the $\Delta S=1$ weak effective Hamiltonian at the next-to-leading order [35]; their numerical values for different choices of $\Lambda_{\overline{\mathrm{MS}}}^{(4)}$ at $\mu=m_{c}$ in the NDR renormalization scheme can be found in [121]. The hadronic parameters $B_{6}^{(1 / 2)}$ and $B_{8}^{(3 / 2)}$ represent the 
matrix elements of the dominant QCD penguin operator $Q_{6}$ and the dominant EW penguin operator $Q_{8}$, respectively. The numerical values of the $P_{i}$ are sensitive functions of $R_{6}$ and $R_{8}$, as well as of $\alpha_{s}$.

On the experimental side, the world average based on the latest results from NA48 [122] and $\mathrm{KTeV}$ [123] and the previous results from NA31 and E731 reads

$$
\varepsilon^{\prime} / \varepsilon=(16.6 \pm 1.6) \times 10^{-4} .
$$

While several analyses made in recent years within the SM found results that are compatible with (5.70), it is fair to say that the large hadronic uncertainties in the coefficients $P_{i}$ still allow for sizeable NP contributions. The relevant list of references can be found in $[121]$.

In the SM, $\theta_{X}=\theta_{Y}=\theta_{Z}=0^{\circ}$ and therefore $\beta_{X}=\beta_{Y}=\beta_{Z}=\beta-\beta_{s}$. We then find [121] that with

$$
P_{0}=19.5, \quad P_{X}=0.6, \quad P_{Y}=0.5, \quad P_{Z}=-12.4, \quad P_{E}=-1.6,
$$

corresponding to $R_{6}=1.2, R_{8}=1.0$ and $\alpha_{s}\left(M_{Z}\right)=0.119$, an agreement with the experimental data can be obtained.

In our scenario, the first term in (5.67) involving $P_{0}$ and originating dominantly in the matrix elements of the QCD penguin operator $Q_{6}$ does not contain any NP contributions, while the important negative last term involving $P_{Z}$ and being related to the EW penguin operator $Q_{8}$ is strongly enhanced. With the values in (5.71), a negative $\varepsilon^{\prime} / \varepsilon$ is then obtained. Thus for our scenario to be consistent with the data, the hadronic matrix element of $Q_{6}$ or equivalently $R_{6}$ must be significantly enhanced over $R_{8}$. For instance for $B_{6}^{(1 / 2)}=2.0, B_{8}^{(3 / 2)}=0.62$ and $m_{s}\left(m_{c}\right)+m_{s}\left(m_{c}\right)=106 \mathrm{MeV}$, corresponding to $R_{6}=2.6$ and $R_{8}=0.81$, one finds for $\alpha_{s}\left(M_{Z}\right)=0.121$ :

$$
P_{0}=48.0, \quad P_{X}=0.65, \quad P_{Y}=0.73, \quad P_{Z}=-10.4, \quad P_{E}=-4.9 .
$$

Setting $\beta_{X}, \beta_{Y}, \beta_{Z}$ at their central values, we obtain

$$
\varepsilon^{\prime} / \varepsilon=15.2 \times 10^{-4},
$$

which is consistent with the experimental data but the result for $\varepsilon^{\prime} / \varepsilon$ is very sensitive to the actual values of the coefficients $P_{i}$ and the angles $\beta_{i}$. As reviewed in [121], $R_{8}=0.8$ used here is consistent with the most recent estimates that give $R_{8}=1.0 \pm 0.2$. Values for $R_{6}$ as high as needed here have been reported in $[124,125]$. In particular the values for $R_{6}=2.2 \pm 0.4$ and $R_{8}=1.1 \pm 0.3$ found in [124], that within the SM would give $\varepsilon^{\prime} / \varepsilon$ substantially higher than the experimental data, would be very welcome within the scenario considered here.

\subsection{Summary}

In this section, we have demonstrated that the sizeably enhanced EW penguins with their large CP-violating NP phase - as implied by the $B \rightarrow \pi K$ data - have important implications for rare $K$ and $B$ decays, as well as for $\varepsilon^{\prime} / \varepsilon$. We find several predictions that differ significantly from the SM expectations and could easily be identified once the data improve. The most interesting results of this study can be summarized as follows: 
- An enhancement of $\operatorname{BR}\left(K_{\mathrm{L}} \rightarrow \pi^{0} \nu \bar{\nu}\right)$ by one order of magnitude without any significant change in $\operatorname{BR}\left(K^{+} \rightarrow \pi^{+} \nu \bar{\nu}\right)$, implying $\operatorname{BR}\left(K_{\mathrm{L}} \rightarrow \pi^{0} \nu \bar{\nu}\right)$ to be close to its absolute upper bound derived in [101].

- A spectacular violation of $(\sin 2 \beta)_{\pi \nu \bar{\nu}}=(\sin 2 \beta)_{\psi K_{\mathrm{S}}}$ [96], which is valid in the SM and any model with minimal flavour violation.

- A large branching ratio $\mathrm{BR}\left(K_{\mathrm{L}} \rightarrow \pi^{0} e^{+} e^{-}\right)=(7.8 \pm 1.6) \times 10^{-11}$, which is governed by direct $\mathrm{CP}$ violation in this scenario, as opposed to the $\mathrm{SM}$, where indirect $\mathrm{CP}$ violation dominates [118].

- A strong enhancement of the integrated forward-backward CP asymmetry for $B_{d} \rightarrow K^{*} \mu^{+} \mu^{-}$.

- Enhancements of $\mathrm{BR}\left(B \rightarrow X_{s, d} \nu \bar{\nu}\right)$ and $\mathrm{BR}\left(B_{s, d} \rightarrow \mu^{+} \mu^{-}\right)$by factors of 2 and 5 , respectively.

- As far as $\varepsilon^{\prime} / \varepsilon$ is concerned, the enhanced EW penguins with their large CPviolating NP phase, as suggested by the $B \rightarrow \pi K$ analysis, require a significant enhancement of the relevant hadronic matrix elements of the QCD penguin operators with respect to the one of the EW penguin operator $Q_{8}$ to be consistent with the $\varepsilon^{\prime} / \varepsilon$ data.

\section{Other Prominent Non-Leptonic B-Meson Decays}

As the last element of our analysis, we explore the implications of our NP scenario of enhanced EW penguins with a new CP-violating weak phase for other prominent nonleptonic $B$-meson decays, which play another key rôle in the physics programme of the $B$ factories.

\section{$6.1 \quad B \rightarrow \phi K$}

In the SM, the $B \rightarrow \phi K$ system is governed by QCD penguins [21] and receives sizeable contributions from EW penguin topologies [22, 23]. Consequently, these modes offer an interesting tool to search for signals of NP, and may of course also be affected within our specific scenario.

\subsubsection{Observables}

In order to address this exciting issue, we follow [29], and consider first

$$
\mathcal{B}_{\phi K} \equiv \frac{1-\mathcal{A}_{\phi K}}{1+\mathcal{A}_{\phi K}},
$$

with

$$
\mathcal{A}_{\phi K} \equiv\left[\frac{\mathrm{BR}\left(B^{+} \rightarrow \phi K^{+}\right)+\mathrm{BR}\left(B^{-} \rightarrow \phi K^{-}\right)}{\operatorname{BR}\left(B_{d}^{0} \rightarrow \phi K^{0}\right)+\operatorname{BR}\left(\bar{B}_{d}^{0} \rightarrow \phi \bar{K}^{0}\right)}\right]\left[\frac{\tau_{B_{d}^{0}}}{\tau_{B^{+}}}\right],
$$


and

$$
\begin{aligned}
& \mathcal{D}_{\phi K}^{-} \equiv \frac{1}{2}\left[\mathcal{A}_{\mathrm{CP}}^{\mathrm{dir}}\left(B_{d} \rightarrow \phi K_{\mathrm{S}}\right)-\mathcal{A}_{\mathrm{CP}}^{\mathrm{dir}}\left(B^{ \pm} \rightarrow \phi K^{ \pm}\right)\right] \\
& \mathcal{D}_{\phi K}^{+} \equiv \frac{1}{2}\left[\mathcal{A}_{\mathrm{CP}}^{\mathrm{dir}}\left(B_{d} \rightarrow \phi K_{\mathrm{S}}\right)+\mathcal{A}_{\mathrm{CP}}^{\mathrm{dir}}\left(B^{ \pm} \rightarrow \phi K^{ \pm}\right)\right] .
\end{aligned}
$$

If we use the experimental results given in [11] and add the errors in quadrature, we obtain

$$
\begin{gathered}
\mathcal{B}_{\phi K}=\left\{\begin{array}{l}
-0.05 \pm 0.10 \text { (BaBar) } \\
+0.06 \pm 0.13 \text { (Belle) }
\end{array}\right. \\
\mathcal{D}_{\phi K}^{-}=\left\{\begin{array}{l}
-0.17 \pm 0.20 \text { (BaBar) } \\
+0.08 \pm 0.16 \text { (Belle) }
\end{array}\right. \\
\mathcal{D}_{\phi K}^{+}=\left\{\begin{array}{l}
-0.21 \pm 0.20 \text { (BaBar) } \\
+0.07 \pm 0.16 \text { (Belle), }
\end{array}\right.
\end{gathered}
$$

whereas the $B$-factory averages correspond to

$$
\mathcal{B}_{\phi K}=0.00 \pm 0.08, \quad \mathcal{D}_{\phi K}^{-}=-0.01 \pm 0.13, \quad \mathcal{D}_{\phi K}^{+}=-0.04 \pm 0.13
$$

We note that these observables are consistent with zero and hence do not indicate any deviation from the SM picture. In particular, as discussed in detail in [29], $\mathcal{B}_{\phi K}$ and $\mathcal{D}_{\phi K}^{-}$ are sensitive to the $I=1$ isospin sector, thereby indicating that the corresponding amplitude $v_{1}$ is in fact suppressed, as is expected on the basis of plausible general arguments. On the other hand, $\mathcal{D}_{\phi K}^{+}$is sensitive to NP in the $I=0$ isospin sector, which involves an amplitude $v_{0}$ with the sine of a CP-conserving strong phase $\Delta_{0}$. The same contribution governs also the difference of $\mathcal{A}_{\mathrm{CP}}^{\operatorname{mix}}\left(B_{d} \rightarrow \phi K_{\mathrm{S}}\right)$ and $\mathcal{A}_{\mathrm{CP}}^{\mathrm{mix}}\left(B_{d} \rightarrow J / \psi K_{\mathrm{S}}\right)$, where it enters, however, with the cosine of $\Delta_{0}$. Within the SM, this difference is expected to vanish to a good approximation [26]-[30], whereas the current $B$-factory data in (1.7) and (1.8) yield

$$
\mathcal{A}_{\mathrm{CP}}^{\mathrm{mix}}\left(B_{d} \rightarrow \phi K_{\mathrm{S}}\right)-\mathcal{A}_{\mathrm{CP}}^{\mathrm{mix}}\left(B_{d} \rightarrow J / \psi K_{\mathrm{S}}\right)=\left\{\begin{array}{l}
+0.3 \pm 0.4 \text { (BaBar) } \\
+1.7 \pm 0.5 \text { (Belle) }
\end{array}\right.
$$

Consequently, we may well arrive at a discrepancy with the SM in the future, although the experimental picture is unclear at the moment. Should such a discrepancy really emerge and $\mathcal{D}_{\phi K}^{+}$still be consistent with zero - in addition to $\mathcal{B}_{\phi K}$ and $\mathcal{D}_{\phi K}^{-}$, we would have an indication for NP in the $I=0$ isospin sector, with a small CP-conserving strong phase $\Delta_{0}$ relative to the SM contribution.

\subsubsection{NP Analysis}

Since our scenario of enhanced EW penguins with a CP-violating weak phase $\phi$ belongs to this category of NP, let us explore its implications in more detail. To this end, we neglect the amplitude $v_{1}$ of the $I=1$ isospin sector, which corresponds to $\mathcal{B}_{\phi K}=\mathcal{D}_{\phi K}^{-}=0$, and write [29]

$$
\begin{aligned}
& A\left(\bar{B}_{d}^{0} \rightarrow \phi \bar{K}^{0}\right)=A_{0}\left[1+v_{0} e^{i\left(\Delta_{0}-\phi\right)}\right]=A\left(B^{-} \rightarrow \phi K^{-}\right) \\
& A\left(B_{d}^{0} \rightarrow \phi K^{0}\right)=A_{0}\left[1+v_{0} e^{i\left(\Delta_{0}+\phi\right)}\right]=A\left(B^{+} \rightarrow \phi K^{+}\right)
\end{aligned}
$$


where the CP-conserving strong amplitude $A_{0}$ describes the QCD penguin contributions, $v_{0}$ measures the strength of the EW penguins with respect to the QCD penguins, which are governed by the $(\bar{s} s)(\bar{b} s)$ quark-flavour structures having $I=0$, and $\Delta_{0}$ is the CP-conserving strong phase mentioned above. Applying now once more the standard formalism for the evaluation of the CP-violating observables, (6.10) and (6.11) yield

$$
\begin{gathered}
\mathcal{A}_{\mathrm{CP}}^{\mathrm{dir}}\left(B_{d} \rightarrow \phi K_{\mathrm{S}}\right)=-\left[\frac{2 v_{0} \sin \Delta_{0} \sin \phi}{1+2 v_{0} \cos \Delta_{0} \cos \phi+v_{0}^{2}}\right]=\mathcal{A}_{\mathrm{CP}}^{\mathrm{dir}}\left(B^{ \pm} \rightarrow \phi K^{ \pm}\right) \\
\mathcal{A}_{\mathrm{CP}}^{\mathrm{mix}}\left(B_{d} \rightarrow \phi K_{\mathrm{S}}\right)=-\left[\frac{\sin \phi_{d}+2 v_{0} \cos \Delta_{0} \sin \left(\phi_{d}+\phi\right)+v_{0}^{2} \sin \left(\phi_{d}+2 \phi\right)}{1+2 v_{0} \cos \Delta_{0} \cos \phi+v_{0}^{2}}\right] .
\end{gathered}
$$

For a simple order-of-magnitude estimate of $v_{0} e^{i \Delta_{0}}$ within the SM, where $\phi=0^{\circ}$, we assume that $A_{0}$ is dominated by internal top-quark exchanges, and use naïve factorization with the leading-order Wilson coefficients given in [126] for $\Lambda_{\frac{(5)}{\mathrm{MS}}}=225 \mathrm{MeV}$. Following these lines, we arrive at

$$
\begin{aligned}
& \left.v_{0} e^{i \Delta_{0}}\right|_{\mathrm{fact}} ^{\mathrm{SM}} \approx-\left[\frac{2\left(C_{9}\left(m_{b}\right)+C_{10}\left(m_{b}\right)\right)}{4\left(C_{3}\left(m_{b}\right)+C_{4}\left(m_{b}\right)\right)+3 C_{5}\left(m_{b}\right)+C_{6}\left(m_{b}\right)}\right] \\
& =-\left[\frac{2 \times(-1.280+0.328)}{4 \times(0.014-0.030)+3 \times 0.009-0.038}\right] \times \frac{1}{128}=-0.20,
\end{aligned}
$$

which corresponds to $\left.\Delta_{0}\right|_{\text {fact }} \approx 180^{\circ}$; for a more refined treatment addressing also QCD penguins with internal up- and charm-quark exchanges, see [22]. In our scenario of NP, the enhancement of $C_{9}+C_{10}$ is the same as that of our EW penguin parameter $q$. Taking into account also the constraints from the rare-decay analysis in Section 5 , we conclude that this enhancement may be at most $\sim 1.3$ with respect to the SM, corresponding to values of $v_{0}$ at the 0.25 level. We may then expand the CP-violating observables in $v_{0}$, which gives

$$
\begin{gathered}
\mathcal{D}_{\phi K}^{+}=-2 v_{0} \sin \Delta_{0} \sin \phi+\mathcal{O}\left(v_{0}^{2}\right) \\
\mathcal{A}_{\mathrm{CP}}^{\operatorname{mix}}\left(B_{d} \rightarrow \phi K_{\mathrm{S}}\right)-\mathcal{A}_{\mathrm{CP}}^{\operatorname{mix}}\left(B_{d} \rightarrow J / \psi K_{\mathrm{S}}\right)=-2 v_{0} \cos \Delta_{0} \sin \phi \cos \phi_{d}+\mathcal{O}\left(v_{0}^{2}\right) .
\end{gathered}
$$

If we use $\cos \phi_{d}=0.68, v_{0} \approx 0.25$, which suffers from large hadronic uncertainties, and take into account that our $B \rightarrow \pi K$ analysis points towards values of $\phi$ around $-90^{\circ}$, corresponding to $\sin \phi \approx-1$, we obtain

$$
\begin{gathered}
\mathcal{D}_{\phi K}^{+} \approx 0.5 \times \sin \Delta_{0} \\
\mathcal{A}_{\mathrm{CP}}^{\text {mix }}\left(B_{d} \rightarrow \phi K_{\mathrm{S}}\right)-\mathcal{A}_{\mathrm{CP}}^{\text {mix }}\left(B_{d} \rightarrow J / \psi K_{\mathrm{S}}\right) \approx 0.3 \times \cos \Delta_{0} .
\end{gathered}
$$

Consequently, the experimental value of $\mathcal{D}_{\phi K}^{+}$in (6.8) favours a small value of $\sin \Delta_{0}$. If we assume that the sign of $\cos \Delta_{0}$ is negative, as in factorization, we conclude that the difference in (6.18) is negative as well. Since in our notation both mixing-induced CP asymmetries would equal $-\sin 2 \beta \approx-0.7$ in the SM, we generically arrive at

$$
(\sin 2 \beta)_{\phi K_{\mathrm{S}}}>(\sin 2 \beta)_{\psi K_{\mathrm{S}}},
$$

where $(\sin 2 \beta)_{\phi K_{\mathrm{S}}}$ around +1 may well be possible, whereas the current result of the Belle collaboration in (1.7) points to a value with the opposite sign around -1 . From the theoretical point of view, there are the following two possible loopholes: 


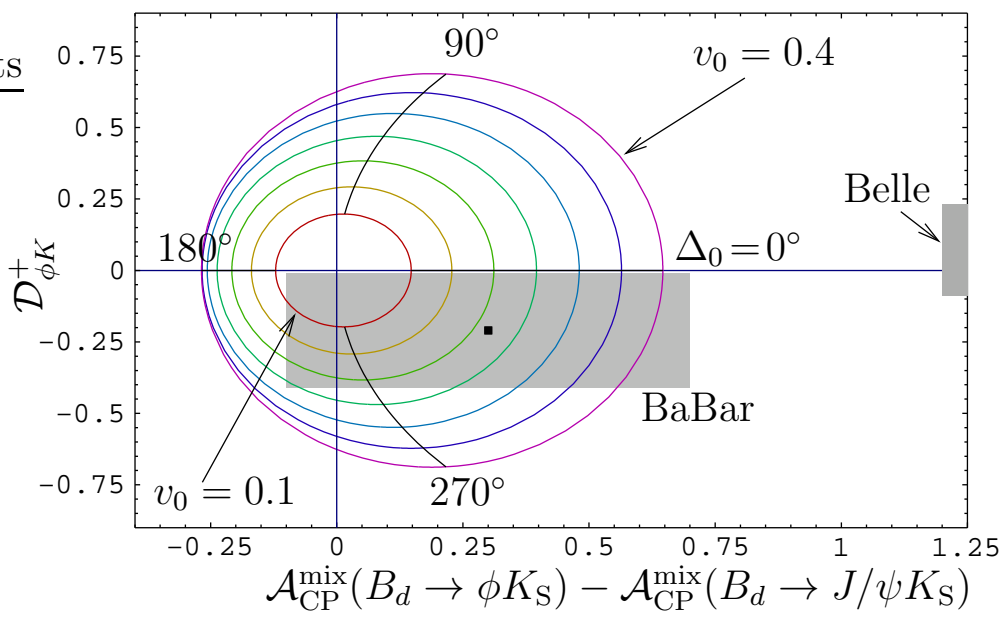

Figure 12: The contours in the plane of $\mathcal{A}_{\mathrm{CP}}^{\operatorname{mix}}\left(B_{d} \rightarrow \phi K_{\mathrm{S}}\right)-\mathcal{A}_{\mathrm{CP}}^{\operatorname{mix}}\left(B_{d} \rightarrow J / \psi K_{\mathrm{S}}\right)$ and $\mathcal{D}_{\phi K}^{+}$for various values of $v_{0}$ with $\Delta_{0} \in\left[0^{\circ}, 360^{\circ}\right]$ and $\phi=-85^{\circ}$, corresponding to the central value of (5.8).

i) In the case of the unconventional solution $\phi_{d}=133^{\circ}$, the sign of $\cos \phi_{d}$ would be negative [55, 56]. However, as we have noted in Subsection 3.4, this possibility appears to be disfavoured.

ii) We may encounter large non-factorizable effects, although it does not look likely that they may flip the sign of $\cos \Delta_{0}$, which would require $\Delta_{0}$ around $0^{\circ}$. On the other hand, for $\Delta_{0}$ around $\pm 90^{\circ}$, the impact of NP on $\mathcal{A}_{\mathrm{CP}}^{\operatorname{mix}}\left(B_{d} \rightarrow \phi K_{\mathrm{S}}\right)$ would be very small, whereas $\mathcal{D}_{\phi K}^{+}$would be large, in conflict with the data.

It is useful to consider the plane of $\mathcal{A}_{\mathrm{CP}}^{\mathrm{mix}}\left(B_{d} \rightarrow \phi K_{\mathrm{S}}\right)-\mathcal{A}_{\mathrm{CP}}^{\operatorname{mix}}\left(B_{d} \rightarrow J / \psi K_{\mathrm{S}}\right)$ and $\mathcal{D}_{\phi K}^{+}$, as done in Fig. 12, where we show also the current experimental results of the BaBar and Belle collaborations. Applying (6.12) and (6.13), we may calculate contours for different values of $v_{0}$, where each point is parametrized by the strong phase $\Delta_{0}$. As far as the NP phase $\phi$ is concerned, we use $\phi=-85^{\circ}$, which is the central value in (5.8). In the future, plots of this kind will allow us to read off the hadronic parameters $v_{0}$ and $\Delta_{0}$ for our NP scenario easily from the improved $B \rightarrow \phi K$ data.

\section{$6.2 \quad B \rightarrow J / \psi K$}

As emphasized in [27], EW penguins may have a non-negligible impact on the $B \rightarrow J / \psi K$ system. Let us, therefore, also address these effects in a more quantitative manner. The starting point is a systematic search for signals of NP arising at the decay-amplitude level of these modes [127], which is - from a formal point of view - completely analogous to the $B \rightarrow \phi K$ system discussed in Subsection 6.1. Using the data for the CP-averaged $B \rightarrow J / \psi K$ branching ratios and the direct CP asymmetries compiled in [11] and [89], respectively, we arrive at the following picture of the $B \rightarrow J / \psi K$ counterparts of (6.1), 
(6.3) and (6.4):

$$
\mathcal{B}_{\psi K}=-0.04 \pm 0.04, \quad \mathcal{D}_{\psi K}^{-}=+0.023 \pm 0.025, \quad \mathcal{D}_{\psi K}^{+}=+0.030 \pm 0.025 .
$$

Consequently, these three observables do not indicate any deviation from the SM.

Within our scenario of NP, we may write the $B \rightarrow J / \psi K$ amplitudes in the same form as (6.10) and (6.11), with expressions for the direct and mixing-induced $\mathrm{CP}$ asymmetries as given in (6.12) and (6.13), respectively. Using again the naïve factorization approach to estimate the relevant EW penguin parameter, we obtain

$$
\begin{gathered}
\left.\left(v_{0} e^{i \Delta_{0}}\right)_{\psi K}\right|_{\mathrm{fact}} ^{\mathrm{SM}} \approx \frac{C_{9}\left(m_{b}\right)+C_{10}\left(m_{b}\right)-\left(1-1 / N_{\mathrm{C}}\right) C_{10}\left(m_{b}\right)}{C_{1}\left(m_{b}\right)+C_{2}\left(m_{b}\right) / N_{\mathrm{C}}} \\
\approx \frac{C_{9}\left(m_{b}\right)+C_{10}\left(m_{b}\right)}{a_{2}^{\psi K}} \approx\left[\frac{-1.280+0.328}{0.25}\right] \times \frac{1}{128} \approx-0.03 .
\end{gathered}
$$

It should be emphasized that this expression suffers from large hadronic uncertainties. In particular, it is very challenging to estimate the effects of QCD penguins to the whole amplitude, which we may consider to be "effectively" included in the phenomenological $B \rightarrow J / \psi K$ colour-suppression factor $a_{2}^{\psi K}$. Nevertheless, it is instructive to consider again an enhancement of $C_{9}+C_{10}$ of 1.3 and a NP phase $\phi$ around $-90^{\circ}$, which yields

$$
\begin{gathered}
\mathcal{D}_{\psi K}^{+} \approx 0.08 \times \sin \left(\Delta_{0}\right)_{\psi K} \\
\mathcal{A}_{\mathrm{CP}}^{\operatorname{mix}}\left(B_{d} \rightarrow J / \psi K_{\mathrm{S}}\right) \approx-\sin 2 \beta+0.05 \times \cos \left(\Delta_{0}\right)_{\psi K},
\end{gathered}
$$

which should be compared with (6.17) and (6.18). Consequently, we may encounter a NP correction to the determination of $\sin 2 \beta$ from the golden mode $B_{d} \rightarrow J / \psi K_{\mathrm{S}}$ at the 0.05 level in our scenario, which corresponds to a shift of at most $\pm 2^{\circ}$ in $\beta$. Such small effects are still beyond the present experimental and theoretical accuracy but could be reinvestigated in the LHC era. Moreover the comparison with similar effects in $\Delta M_{s, d}$ could, in the future, shed some light on the size of the parameter $\left(v_{0} e^{i \Delta_{0}}\right)_{\psi K}$. It should also be noted that this parameter is expected to be different for higher $J / \psi$ resonances, so that the NP effects may largely cancel in the averages over such modes, which are usually formed by the BaBar and Belle collaborations and are also at the basis of (1.8).

In order to search for NP effects in the "golden" mode $B_{d} \rightarrow J / \psi K_{\mathrm{S}}$, decays of the kind $B_{d} \rightarrow D \pi^{0}, D \rho^{0}, \ldots$ are particularly interesting. If the neutral $D$ mesons are observed through their decays into CP eigenstates $D_{ \pm}$, these pure "tree" decays, which do not receive any penguin contributions, allow extremely clean determinations of $\sin 2 \beta$ [128]. Consequently, a future tiny discrepancy with $(\sin 2 \beta)_{\psi K_{\mathrm{S}}}$ could also provide valuable insights into our specific scenario of NP.

\subsection{Summary}

The main results of our analysis of the $B \rightarrow \phi K$ and $B \rightarrow J / \psi K$ systems can be summarized as follows: 
- Within our NP scenario, we expect generically $(\sin 2 \beta)_{\phi K_{\mathrm{S}}}>(\sin 2 \beta)_{\psi K_{\mathrm{S}}}$, where $(\sin 2 \beta)_{\phi K_{\mathrm{S}}} \sim+1$ may well be possible. This pattern is qualitatively different from the current $B$-factory data, which are, however, not yet conclusive. On the other hand, a future confirmation of this pattern would be another signal of enhanced CP-violating EW penguins at work.

- The measurement of $\sin 2 \beta$ through the golden mode $B_{d} \rightarrow J / \psi K_{\mathrm{S}}$ may receive NP corrections as large as 0.05 , which are, however, affected by large - essentially unknown - hadronic effects. The current $B$-factory data disfavour an enhancement of the relevant NP parameter through such effects.

\section{Conclusions}

In this paper, we have developed a strategy that allows us to study simultaneously nonleptonic two-body $B$ decays and rare $K$ and $B$ decays within the SM and its simple extension in which the dominant NP effects come from modified $Z^{0}$-penguin contributions with a new CP-violating weak phase. This simple scenario is parametrized by only two variables $(q, \phi)$ that take the values $q=0.69$ and $\phi=0^{\circ}$ in the SM.

The aim of our study was not only to learn about possible NP effects in the processes in question but also to determine the angle $\gamma$ and to gain some insight into the hadron dynamics, which plays an important rôle in non-leptonic $B$ decays and $\varepsilon^{\prime} / \varepsilon$, is less important in decays such as $B \rightarrow X_{s} \mu^{+} \mu^{-}$, and is essentially irrelevant in theoretically clean processes such as $K \rightarrow \pi \nu \bar{\nu}$.

If we had at our disposal precise experimental data for all processes considered here, the most efficient strategy would be to choose decays that are theoretically clean and free from NP contributions to determine the angle $\gamma$, use then this value in clean rare decays sensitive to NP in order to determine $(q, \phi)$, and finally use $\gamma$ and $(q, \phi)$ in processes sensitive to hadron dynamics with the aim to obtain insight into the latter. In order to implement this procedure, clean tree-level strategies for the determination of $\gamma$ (see $[2,72,128]$ and references therein), the rare decays $K \rightarrow \pi \nu \bar{\nu}$, and non-leptonic decays such as $B \rightarrow \pi \pi$ and $B \rightarrow \pi K$ could be used, respectively.

Unfortunately, at present, the first two steps of this strategy cannot be made in view of the lack of data required for the clean strategies for $\gamma$ in question and in view of the insufficient experimental information on $K \rightarrow \pi \nu \bar{\nu}$ decays. On the other hand, data on $B \rightarrow \pi \pi$ and $B \rightarrow \pi K$ are already available. Even though they are not yet very accurate, the progress expected in the coming years in measuring the relevant observables at the $B$ factories allows us to expect that these non-leptonic decays will be known at an acceptable precision well before $K \rightarrow \pi \nu \bar{\nu}$ decays - in particular $K_{\mathrm{L}} \rightarrow \pi^{0} \nu \bar{\nu}$ - and clean tree-level strategies for $\gamma$ will become useful.

In view of this situation, it is necessary to concentrate first on the usual UT fits for $\gamma, B \rightarrow \pi \pi$ and $B \rightarrow \pi K$ decays, determine $\gamma, q, \phi$ and hadronic parameters by using appropriate observables and $S U(3)$ flavour-symmetry and plausible dynamical assumptions, and subsequently make predictions for rare decays. The three-step procedure for achieving this goal was presented in Section 1 and executed in the rest of the paper. 
Our analysis has been summarized briefly in [12] and presented in detail in Sections 36 of the present work. A list of results can be found at the end of each section. Here we recall the most important findings of our "anatomy":

- Using the available data for $B \rightarrow \pi \pi$ decays and neglecting small EW penguin contributions to the relevant decay amplitudes, we were able to determine four hadronic parameters using only the isospin symmetry of strong interactions and the information for the angle $\gamma$ from the UT fits. The result is given in (3.41) and (3.42). This determination is essentially free from theoretical uncertainties and the large errors in (3.41) and (3.42), which reflect the large experimental uncertainties in $R_{+-}^{\pi \pi}, R_{00}^{\pi \pi}, \mathcal{A}_{\mathrm{CP}}^{\text {dir }}\left(B_{d} \rightarrow \pi^{+} \pi^{-}\right)$and $\mathcal{A}_{\mathrm{CP}}^{\text {mix }}\left(B_{d} \rightarrow \pi^{+} \pi^{-}\right)$, should be significantly reduced in the coming years. Contour plots in Figs. 1 and 2 will allow a monitoring of these improvements.

- Having the determined hadronic parameters of the $B \rightarrow \pi \pi$ system at hand, we may predict the $\mathrm{CP}$-violating observables of the $B_{d} \rightarrow \pi^{0} \pi^{0}$ channel as given in (3.71) and (3.72), with the interesting perspective of having large direct and mixinginduced $\mathrm{CP}$ violation in this decay. Moreover, if at least one of these observables is measured, $\gamma$ can be determined in a theoretically clean way.

- If we make the plausible assumption that penguin annihilation and exchange topologies play a negligible rôle and employ the $S U(3)$ flavour symmetry of strong interactions, we may complement the CP-violating observables of $B_{d} \rightarrow \pi^{+} \pi^{+}$in a variety of ways with the data provided by the $B_{d} \rightarrow \pi^{\mp} K^{ \pm}$modes, which are only insignificantly affected by EW penguin contributions. Following these lines, we may extract $\gamma$, as given in (3.64). This determination can be refined with the help of other $B \rightarrow \pi K$ observables, with the result in (4.29), which is in very good agreement with the UT fits, as can be seen in Fig. 8. The remarkably consistent overall picture of our analysis also indicates that non-factorizable $S U(3)$-breaking effects are moderate and that our other dynamical assumptions are justified.

- Using the hadronic $B \rightarrow \pi \pi$ parameters determined in the first step of our strategy, we may fix their $B \rightarrow \pi K$ counterparts with the help of the $S U(3)$ flavour symmetry and plausible dynamical assumptions (see previous item), allowing us to analyse the $B \rightarrow \pi K$ system. Interestingly, we find that the SM cannot properly describe those $B \rightarrow \pi K$ observables that are sensitive to EW penguin contributions. This is in particular the case for $R_{\mathrm{c}}$ and $R_{\mathrm{n}}$, for which we obtain $R_{\mathrm{c}} \sim 1.14$ and $R_{\mathrm{n}} \sim 1.11$ in the SM, whereas experiments give $R_{\mathrm{c}} \sim 1.17$ and $R_{\mathrm{n}} \sim 0.76$. On the other hand, the pattern of the $B$-factory data for those $B \rightarrow \pi K$ observables that are only insignificantly affected by EW penguins does not show any anomalous behaviour, i.e. is in accordance with the SM picture.

- We have demonstrated that all features of the present $B \rightarrow \pi K$ data can be described within a NP scenario, where EW penguin topologies are moderatly enhanced and carry a large CP-violating NP phase $\phi$ around $-90^{\circ}$, thereby requiring new sources for $\mathrm{CP}$ violation that lie beyond the KM mechanism. Of particular interest are our predictions for $\mathcal{A}_{\mathrm{CP}}^{\mathrm{dir}}\left(B_{d} \rightarrow \pi^{0} K_{\mathrm{S}}\right)$ and $\mathcal{A}_{\mathrm{CP}}^{\text {mix }}\left(B_{d} \rightarrow \pi^{0} K_{\mathrm{S}}\right)$, and the 
suggestion to use these observables in the future for the determination of the parameters of the EW penguin sector. Moreover, also studies of $B_{s} \rightarrow K^{+} K^{-}$and $B_{s} \rightarrow \pi^{ \pm} K^{\mp}$ decays, which are very accessible at LHCb, complement our strategy nicely. We made also predictions for the corresponding $B_{s}$-decay observables.

- Restricting ourselves to a more specific NP scenario with enhanced $Z^{0}$ penguins, we may explore the implications of our $B \rightarrow \pi K$ analysis for rare $K$ and $B$ decays, where in particular the CP-violating NP phase $\phi$ has important consequences. In turn, the currently available rare-decay data have already some impact on the allowed ranges for the $B \rightarrow \pi K$ observables as summarized in Table 2. A detailed analysis of these effects has been presented in Section 5. Possibly the most interesting effects are found in the $K \rightarrow \pi \nu \bar{\nu}$ system, where the enhancement of $\mathrm{BR}\left(K_{\mathrm{L}} \rightarrow \pi^{0} \nu \bar{\nu}\right)$ by one order of magnitude and a strong violation of the MFV relation $(\sin 2 \beta)_{\pi \nu \bar{\nu}}=(\sin 2 \beta)_{\psi K_{\mathrm{S}}}$ are very spectacular. Similarly to the asymmetries $\mathcal{A}_{\mathrm{CP}}^{\mathrm{dir}}\left(B_{d} \rightarrow \pi^{0} K_{\mathrm{S}}\right)$ and $\mathcal{A}_{\mathrm{CP}}^{\mathrm{mix}}\left(B_{d} \rightarrow \pi^{0} K_{\mathrm{S}}\right)$, the branching ratios for $K^{+} \rightarrow \pi^{+} \nu \bar{\nu}$ and $K_{\mathrm{L}} \rightarrow \pi^{0} \nu \bar{\nu}$ will allow a useful determination of the EW penguin parameters and the comparison of these two very different determinations will be a very important test of the NP scenario considered here.

- Finally, as discussed in Section 6, we expect a drastic modification of the Belle result $(\sin 2 \beta)_{\psi K_{\mathrm{S}}} \gg(\sin 2 \beta)_{\phi K_{\mathrm{S}}}$, in that we find $(\sin 2 \beta)_{\phi K_{\mathrm{S}}} \gtrsim(\sin 2 \beta)_{\psi K_{\mathrm{S}}}$. Consequently, within the NP scenario considered here, we find $(\sin 2 \beta)_{\phi K_{\mathrm{S}}}$ to be of the same magnitude as the central value found by the Belle collaboration but of opposite sign!

As we have seen in our analysis, studies of $B \rightarrow \pi \pi$ and $B \rightarrow \pi K$ decays are not only interesting in the context of the exploration of $\mathrm{CP}$ violation and the search for NP, but also to obtain valuable insights into hadron physics. Consequently, improved measurements of the corresponding observables are also very important in order to see whether the theoretical approaches like QCDF [10], PQCD [129] and SCET [130], in addition to their interesting theoretical structures, are also phenomenologically useful. Independently of the outcome of these measurements, the phenomenological strategy presented here will be very useful in correlating the experimental results for $B \rightarrow \pi \pi$ and $B \rightarrow \pi K$ with those for rare $K$ and $B$ decays, $B_{d} \rightarrow \phi K_{\mathrm{S}}$ and $\varepsilon^{\prime} / \varepsilon$.

Assuming that future, more accurate $B \rightarrow \pi \pi, \pi K$ data will not modify significantly the currently observed pattern in these decays, the scenario of enhanced $Z^{0}$ penguins with a large NP phase will remain an attractive possibility. While the enhancement of $\mathrm{BR}\left(K_{\mathrm{L}} \rightarrow \pi^{0} \nu \bar{\nu}\right)$ by one order of magnitude would be very welcome to our experimental colleagues and $(\sin 2 \beta)_{\pi \nu \bar{\nu}}<0$ would be a very spectacular signal of NP, even more moderate departures of this sort from the SM and the MFV expectations could be easily identified in the very clean $K \rightarrow \pi \nu \bar{\nu}$ decays as clear signals of NP.

\section{Acknowledgements}

The work presented here was supported in part by the German Bundesministerium für Bildung und Forschung under the contract 05HT1WOA3 and the DFG Project Bu. 706/1-2. 


\section{A Compendium}

\section{A.1 The $B \rightarrow \pi \pi$ System}

$$
\begin{gathered}
R_{+-}^{\pi \pi}=\frac{1+2 x \cos \Delta+x^{2}}{1-2 d \cos \theta \cos \gamma+d^{2}} \\
R_{00}^{\pi \pi}=\frac{d^{2}+2 d x \cos (\Delta-\theta) \cos \gamma+x^{2}}{1-2 d \cos \theta \cos \gamma+d^{2}} \\
\mathcal{A}_{\mathrm{CP}}^{\text {dir }}\left(B_{d} \rightarrow \pi^{+} \pi^{-}\right)=-\left[\frac{2 d \sin \theta \sin \gamma}{1-2 d \cos \theta \cos \gamma+d^{2}}\right] \\
\mathcal{A}_{\mathrm{CP}}^{\operatorname{mix}}\left(B_{d} \rightarrow \pi^{+} \pi^{-}\right)=\frac{\sin \left(\phi_{d}+2 \gamma\right)-2 d \cos \theta \sin \left(\phi_{d}+\gamma\right)+d^{2} \sin \phi_{d}}{1-2 d \cos \theta \cos \gamma+d^{2}} \\
\mathcal{A}_{\mathrm{CP}}^{\operatorname{dir}}\left(B_{d} \rightarrow \pi^{0} \pi^{0}\right)=\frac{2 d x \sin (\theta-\Delta) \sin \gamma}{d^{2}+2 d x \cos (\theta-\Delta) \cos \gamma+x^{2}} \\
\mathcal{A}_{\mathrm{CP}}^{\operatorname{mix}}\left(B_{d} \rightarrow \pi^{0} \pi^{0}\right)=\frac{d^{2} \sin \phi_{d}+2 d x \cos (\theta-\Delta) \sin \left(\phi_{d}+\gamma\right)+x^{2} \sin \left(\phi_{d}+2 \gamma\right)}{d^{2}+2 d x \cos (\theta-\Delta) \cos \gamma+x^{2}} .
\end{gathered}
$$

\section{A.2 The $B \rightarrow \pi K$ System}

$$
\begin{aligned}
& R=1-2 r \cos \delta \cos \gamma+r^{2} \\
& R_{\mathrm{c}}=1-2 r_{\mathrm{c}} \cos \delta_{\mathrm{c}} \cos \gamma+r_{\mathrm{c}}^{2} \\
& +q r_{\mathrm{c}}\left[2\left\{\cos \left(\delta_{\mathrm{c}}+\omega\right) \cos \phi-r_{\mathrm{c}} \cos \omega \cos (\gamma-\phi)\right\}+q r_{\mathrm{c}}\right] \\
& R_{\mathrm{n}}=\frac{1}{b}\left[1-2 r \cos \delta \cos \gamma+r^{2}\right] \\
& b \equiv \frac{R}{R_{\mathrm{n}}}=1-2 q r_{\mathrm{c}} \cos \left(\delta_{\mathrm{c}}+\omega\right) \cos \phi+q^{2} r_{\mathrm{c}}^{2} \\
& +2 \rho_{\mathrm{n}}\left[\cos \theta_{\mathrm{n}} \cos \gamma-q r_{\mathrm{c}} \cos \left(\theta_{\mathrm{n}}-\delta_{\mathrm{c}}-\omega\right) \cos (\gamma-\phi)\right]+\rho_{\mathrm{n}}^{2} \\
& \mathcal{A}_{\mathrm{CP}}^{\mathrm{dir}}\left(B_{d} \rightarrow \pi^{\mp} K^{ \pm}\right)=\frac{2 r \sin \delta \sin \gamma}{1-2 r \cos \delta \cos \gamma+r^{2}} \\
& \mathcal{A}_{\mathrm{CP}}^{\operatorname{dir}}\left(B^{ \pm} \rightarrow \pi^{ \pm} K\right)=-\left[\frac{2 \rho_{\mathrm{c}} \sin \theta_{\mathrm{c}} \sin \gamma}{1+2 \rho_{\mathrm{c}} \cos \theta_{\mathrm{c}} \cos \gamma+\rho_{\mathrm{c}}^{2}}\right] \\
& \mathcal{A}_{\mathrm{CP}}^{\mathrm{dir}}\left(B^{ \pm} \rightarrow \pi^{0} K^{ \pm}\right)=\frac{2}{R_{\mathrm{c}}}\left[r_{\mathrm{c}} \sin \delta_{\mathrm{c}} \sin \gamma-q r_{\mathrm{c}}\left\{\sin \left(\delta_{\mathrm{c}}+\omega\right) \sin \phi+r_{\mathrm{c}} \sin \omega \sin (\gamma-\phi)\right\}\right] \\
& \mathcal{A}_{\mathrm{CP}}^{\mathrm{dir}}\left(B_{d} \rightarrow \pi^{0} K_{\mathrm{S}}\right)=\frac{2}{b}\left[q r_{\mathrm{c}} \sin \left(\delta_{\mathrm{c}}+\omega\right) \sin \phi\right. \\
& \left.-\rho_{\mathrm{n}}\left\{\sin \theta_{\mathrm{n}} \sin \gamma-q r_{\mathrm{c}} \sin \left(\theta_{\mathrm{n}}-\delta_{\mathrm{c}}-\omega\right) \sin (\gamma-\phi)\right\}\right]
\end{aligned}
$$




$$
\begin{gathered}
\mathcal{A}_{\mathrm{CP}}^{\mathrm{mix}}\left(B_{d} \rightarrow \pi^{0} K_{\mathrm{S}}\right)=-\frac{1}{b}\left[\sin \phi_{d}-2 q r_{\mathrm{c}} \cos \left(\delta_{\mathrm{c}}+\omega\right) \sin \left(\phi_{d}+\phi\right)+q^{2} r_{\mathrm{c}}^{2} \sin \left(\phi_{d}+2 \phi\right)(\mathrm{A} .15)\right. \\
\left.+2 \rho_{\mathrm{n}}\left\{\cos \theta_{\mathrm{n}} \sin \left(\phi_{d}+\gamma\right)-q r_{\mathrm{c}} \cos \left(\theta_{\mathrm{n}}-\delta_{\mathrm{c}}-\omega\right) \sin \left(\phi_{d}+\gamma+\phi\right)\right\}+\rho_{\mathrm{n}}^{2} \sin \left(\phi_{d}+2 \gamma\right)\right] .
\end{gathered}
$$

\section{A.3 The $B_{s} \rightarrow K^{+} K^{-}, B_{s} \rightarrow \pi^{ \pm} K^{\mp}$ System}

Using the $U$-spin flavour symmetry of strong interactions, we may express the corresponding CP-violating observables in terms of the hadronic $B_{d} \rightarrow \pi^{+} \pi^{-}$and $B_{d} \rightarrow \pi^{\mp} K^{ \pm}$ parameters as follows:

$$
\begin{gathered}
\mathcal{A}_{\mathrm{CP}}^{\mathrm{dir}}\left(B_{s} \rightarrow K^{+} K^{-}\right)=\frac{2 \epsilon d \sin \theta \sin \gamma}{\epsilon^{2}+2 \epsilon d \cos \theta \cos \gamma+d^{2}} \\
\mathcal{A}_{\mathrm{CP}}^{\mathrm{mix}}\left(B_{s} \rightarrow K^{+} K^{-}\right)=\frac{\epsilon^{2} \sin \left(\phi_{s}+2 \gamma\right)+2 \epsilon d \cos \theta \sin \left(\phi_{s}+\gamma\right)+d^{2} \sin \phi_{s}}{\epsilon^{2}+2 \epsilon d \cos \theta \cos \gamma+d^{2}} \\
\mathcal{A}_{\mathrm{CP}}^{\mathrm{dir}}\left(B_{s} \rightarrow \pi^{ \pm} K^{\mp}\right)=-\left[\frac{2 \epsilon r \sin \delta \sin \gamma}{\epsilon^{2}+2 \epsilon r \cos \delta \cos \gamma+r^{2}}\right] .
\end{gathered}
$$

\section{B Comment on the New Belle $B_{d} \rightarrow \pi^{+} \pi^{-}$Results}

During the final stages of this work, the Belle collaboration announced the following update of the results for the CP-violating observables of the $B_{d} \rightarrow \pi^{+} \pi^{-}$channel [67]:

$$
\begin{aligned}
& \mathcal{A}_{\mathrm{CP}}^{\mathrm{dir}}\left(B_{d} \rightarrow \pi^{+} \pi^{-}\right)=-0.58 \pm 0.15 \pm 0.07 \\
& \mathcal{A}_{\mathrm{CP}}^{\mathrm{mix}}\left(B_{d} \rightarrow \pi^{+} \pi^{-}\right)=+1.00 \pm 0.21 \pm 0.07
\end{aligned}
$$

Using these new data, the averages in (3.29) and (3.30) change to

$$
\begin{aligned}
& \mathcal{A}_{\mathrm{CP}}^{\mathrm{dir}}\left(B_{d} \rightarrow \pi^{+} \pi^{-}\right)=-0.42 \pm 0.13 \\
& \mathcal{A}_{\mathrm{CP}}^{\mathrm{mix}}\left(B_{d} \rightarrow \pi^{+} \pi^{-}\right)=+0.70 \pm 0.19 .
\end{aligned}
$$

Whereas the global picture of the analysis presented in this paper is not affected by these new numbers, some numerical results change. In particular, instead of (3.64), the averages in (B.3) and (B.4) would correspond to the following smaller values of $\gamma$ :

$$
\gamma=\left(38.6_{-7.2}^{+6.1}\right)^{\circ} \vee\left(55.6_{-8.1}^{+7.0}\right)^{\circ}
$$

which would also be in accordance with UT fits, although a bit on the lower side. The contour corresponding to the new values was added to Fig. 8, and the central values in (4.31) change to $\alpha=103.3^{\circ}$ and $\beta=21.1^{\circ}$. 


\section{Error Treatment}

Unless otherwise stated (e.g. in the determination of $\gamma$ in Subsection 3.4), we treat the errors in the following way: all predicted quantities depend on the hadronic parameters $d, \theta, x$ and $\Delta$, and on some other input parameters. To take into account the fact that dependences on the hadronic parameters can cancel out, we do not vary the hadronic parameters inside their error bands, but rather vary the input parameters that are used to obtain them, i.e. $\mathcal{A}_{\mathrm{CP}}^{\mathrm{dir}}\left(B_{d} \rightarrow \pi^{+} \pi^{-}\right), \mathcal{A}_{\mathrm{CP}}^{\mathrm{mix}}\left(B_{d} \rightarrow \pi^{+} \pi^{-}\right), R_{+-}^{\pi \pi}$ and $R_{00}^{\pi \pi}$. Other input parameters that contribute to the errors are $\gamma$ and $\phi_{d}$, as well as $A$ and additional hadronic parameters appearing in the context of Section 5 (some quantities involve also $R_{b}$ ). The individual errors associated with the uncertainty of a specific input parameter are found by varying the corresponding parameter within its $1 \sigma$ band, while keeping the other parameters fixed at their central values. These errors are then added up in quadrature to obtain the total error for each observable.

\section{Colour-Suppressed EW Penguins in $B \rightarrow \pi K$}

\section{D.1 General Structure}

In order to discuss the EW penguin contributions to the decays $B^{+} \rightarrow \pi^{+} K^{0}$ and $B_{d}^{0} \rightarrow \pi^{-} K^{+}$, which are usually referred to as "colour-suppressed", we have to look at the EW penguin operators, exhibiting the following generic flavour structure:

$$
\mathcal{Q}_{\mathrm{EW}}^{\mathrm{pen}} \sim \frac{1}{2}[2(\bar{c} c)-(\bar{s} s)-(\bar{b} b)+\{2(\bar{u} u)-(\bar{d} d)\}](\bar{b} s) .
$$

Using then the well-known isospin decomposition

$$
(\bar{u} u)=\frac{1}{2} \underbrace{[(\bar{u} u)+(\bar{d} d)]}_{I=0}+\frac{1}{2} \underbrace{[(\bar{u} u)-(\bar{d} d)]}_{I=1},
$$

we may decompose the EW penguin operators into isospin singlet and triplet pieces as follows:

$$
\begin{aligned}
\left(\mathcal{Q}_{\mathrm{EW}}^{\mathrm{pen}}\right)_{I=0} \sim & \frac{1}{2}\left[2(\bar{c} c)-(\bar{s} s)-(\bar{b} b)+\frac{1}{2}\{(\bar{u} u)+(\bar{d} d)\}\right](\bar{b} s) \\
& \left(\mathcal{Q}_{\mathrm{EW}}^{\mathrm{pen}}\right)_{I=1} \sim \frac{3}{4}[(\bar{u} u)-(\bar{d} d)](\bar{b} s) .
\end{aligned}
$$

If we then apply the isospin flavour symmetry of strong interactions, as discussed in detail in [62], we obtain ${ }^{13}$

$$
\begin{aligned}
& A\left(B^{+} \rightarrow \pi^{+} K^{0}\right)_{\mathrm{EW}}=+\frac{1}{2}\left[P_{\mathrm{EW}}^{\mathrm{C}(0)}-P_{\mathrm{EW}}^{\mathrm{C}(1)}\right] \equiv-\frac{1}{3} P_{\mathrm{EW}}^{\mathrm{C}-} \\
& A\left(B_{d}^{0} \rightarrow \pi^{-} K^{+}\right)_{\mathrm{EW}}=-\frac{1}{2}\left[P_{\mathrm{EW}}^{\mathrm{C}(0)}+P_{\mathrm{EW}}^{\mathrm{C}(1)}\right] \equiv-\frac{2}{3} P_{\mathrm{EW}}^{\mathrm{C}+},
\end{aligned}
$$

\footnotetext{
${ }^{13}$ For simplicity, we suppress in the following discussion the primes introduced in Section 4 to distinguish the $B \rightarrow \pi K$ amplitudes from their $B \rightarrow \pi \pi$ counterparts.
} 
with

$$
\begin{gathered}
P_{\mathrm{EW}}^{\mathrm{C}(0)}=-\frac{G_{\mathrm{F}}}{\sqrt{2}} \lambda^{2} A \frac{1}{2} C\left\langle K^{+} \pi^{-}|[4(\bar{c} c)-2(\bar{s} s)-2(\bar{b} b)+\{(\bar{u} u)+(\bar{d} d)\}](\bar{b} s)| B_{d}^{0}\right\rangle \\
P_{\mathrm{EW}}^{\mathrm{C}(1)}=-\frac{G_{\mathrm{F}}}{\sqrt{2}} \lambda^{2} A \frac{3}{2} C\left\langle K^{+} \pi^{-}|[(\bar{u} u)-(\bar{d} d)](\bar{b} s)| B_{d}^{0}\right\rangle,
\end{gathered}
$$

summarizing the isospin singlet and triplet pieces, respectively. Here the combination of the generic Wilson coefficient $C$ with the four-quark operators denotes symbolically the sum over the relevant EW penguin operators. We observe that

$$
A\left(B^{+} \rightarrow \pi^{+} K^{0}\right)+A\left(B_{d}^{0} \rightarrow \pi^{-} K^{+}\right)=-P_{\mathrm{EW}}^{\mathrm{C}(1)},
$$

where the expression for $P_{\mathrm{EW}}^{\mathrm{C}(1)}$ in (D.8) agrees with the one for the colour-suppressed EW penguin amplitude introduced in [46].

It is instructive to consider the tree-diagram-like matrix elements entering (D.7) and (D.8), which read as follows:

$$
\begin{aligned}
& {\left[P_{\mathrm{EW}}^{\mathrm{C}(0)}\right]_{\mathrm{T}}=-\frac{G_{\mathrm{F}}}{\sqrt{2}} \lambda^{2} A \frac{1}{2} C\left\langle K^{+} \pi^{-}|(\bar{u} u)(\bar{b} s)| B_{d}^{0}\right\rangle_{\mathrm{T}}} \\
& {\left[P_{\mathrm{EW}}^{\mathrm{C}(1)}\right]_{\mathrm{T}}=-\frac{G_{\mathrm{F}}}{\sqrt{2}} \lambda^{2} A \frac{3}{2} C\left\langle K^{+} \pi^{-}|(\bar{u} u)(\bar{b} s)| B_{d}^{0}\right\rangle_{\mathrm{T}},}
\end{aligned}
$$

and imply

$$
\left[P_{\mathrm{EW}}^{\mathrm{C}-\mathrm{T}}\right]_{\mathrm{T}}=\left[P_{\mathrm{EW}}^{\mathrm{C}+}\right]_{\mathrm{T}}=\left[P_{\mathrm{EW}}^{\mathrm{C}(1)}\right]_{\mathrm{T}} .
$$

The EW penguin operator $Q_{9}$ with the largest Wilson coefficient has the colour structure $\left(\bar{u}_{\alpha} u_{\alpha}\right)\left(\bar{b}_{\beta} s_{\beta}\right)$ and its matrix elements (D.10) and (D.11) are consequently coloursuppressed. On the other hand, $Q_{10} \sim\left(\bar{u}_{\alpha} u_{\beta}\right)\left(\bar{b}_{\beta} s_{\alpha}\right)$ has a significantly smaller Wilson coefficient. The coefficients of the $(V-A) \otimes(V+A)$ EW penguin operators $Q_{7}$ and $Q_{8}$ are even further suppressed, so that these operators can be neglected. Consequently, the hadronic matrix elements in (D.10) and (D.11) are colour-suppressed, and (D.12) represents the picture of the colour-suppressed EW penguins that is usually adopted in the literature.

In our analysis of the $B \rightarrow \pi \pi$ system in Section 3, we have seen that penguin topologies with internal up- and charm-quark exchanges, which correspond to matrix elements with penguin-like contractions of $(\bar{u} u)(\bar{b} s)$ and $(\bar{c} c)(\bar{b} s)$ operators with $u$ and $c$ quarks running in the loops [62], play an important rôle. Consequently, the standard picture of colour-suppressed EW penguins in $B \rightarrow \pi K$ could also be affected through these penguin topologies. Moreover, the parameters

$$
\begin{gathered}
\tilde{a}_{\mathrm{C}} e^{i \tilde{\Delta}_{\mathrm{C}}} \equiv \frac{P_{\mathrm{EW}}^{\mathrm{C}-}}{P_{\mathrm{EW}}}=\frac{P_{\mathrm{EW}}^{\mathrm{C}-}}{P_{\mathrm{EW}}^{\mathrm{C}-}+\tilde{P}_{\mathrm{EW}}^{\mathrm{A}}} \\
a_{\mathrm{C}} e^{i \Delta_{\mathrm{C}}} \equiv \frac{P_{\mathrm{EW}}^{\mathrm{C}+}}{P_{\mathrm{EW}}}=\frac{P_{\mathrm{EW}}^{\mathrm{C}+}}{P_{\mathrm{EW}}^{\mathrm{C}+}+P_{\mathrm{EW}}^{\mathrm{A}}},
\end{gathered}
$$


where $\tilde{P}_{\mathrm{EW}}^{\mathrm{A}}$ and $P_{\mathrm{EW}}^{\mathrm{A}}$ are the colour-allowed $\mathrm{EW}$ penguin amplitudes contributing to $\sqrt{2} A\left(B^{+} \rightarrow \pi^{0} K^{+}\right)$and $\sqrt{2} A\left(B_{d}^{0} \rightarrow \pi^{0} K^{0}\right)$, respectively, may not be as small as naïvely expected. In (D.13) and (D.14), we have used that the isospin flavour symmetry implies

$$
P_{\mathrm{EW}}=P_{\mathrm{EW}}^{\mathrm{C}-}+\tilde{P}_{\mathrm{EW}}^{\mathrm{A}}=P_{\mathrm{EW}}^{\mathrm{C}+}+P_{\mathrm{EW}}^{\mathrm{A}},
$$

where $P_{\mathrm{EW}}$ enters the EW penguin parameter $q e^{i \phi} e^{i \omega}$. As in Subsection 2.3, we define the weak phases of $P_{\mathrm{EW}}^{\mathrm{C}-}$ and $P_{\mathrm{EW}}^{\mathrm{C}+}$ as the one associated with the combination $C_{9}+C_{10}$ of Wilson coefficients, which can be done approximately.

In the remainder of this appendix, we discuss in detail the possible impact of the parameters in (D.13) and (D.14) on the $B \rightarrow \pi K$ analysis performed in Section 4, and propose strategies to search for indications of their effects in the corresponding data.

\section{D.2 Generalization of the Decay Amplitudes}

The generalization of the decay amplitudes in (4.1)-(4.4), taking the colour-suppressed EW penguin topologies into account, is given by

$$
\begin{aligned}
A\left(B^{+} \rightarrow \pi^{+} K^{0}\right) & =-P^{\prime}\left[1+\rho_{\mathrm{c}} e^{i \theta_{\mathrm{c}}} e^{i \gamma}-\frac{1}{3} \tilde{a}_{\mathrm{EW}}^{\mathrm{C}} e^{i \tilde{\psi}_{\mathrm{C}}} e^{i \phi}\right] \\
\sqrt{2} A\left(B^{+} \rightarrow \pi^{0} K^{+}\right) & =P^{\prime}\left[1+\rho_{\mathrm{c}} e^{i \theta_{\mathrm{c}}} e^{i \gamma}-\left\{e^{i \gamma}-\left(1-\frac{1}{3} \tilde{a}_{\mathrm{C}} e^{i \tilde{\Delta}_{\mathrm{C}}}\right) q e^{i \phi} e^{i \omega}\right\} r_{\mathrm{c}} e^{i \delta_{\mathrm{c}}}\right] \\
A\left(B_{d}^{0} \rightarrow \pi^{-} K^{+}\right) & =P^{\prime}\left[1+\frac{2}{3} a_{\mathrm{EW}}^{\mathrm{C}} e^{i \psi_{\mathrm{C}}} e^{i \phi}-r e^{i \delta} e^{i \gamma}\right] \\
\sqrt{2} A\left(B_{d}^{0} \rightarrow \pi^{0} K^{0}\right) & =-P^{\prime}\left[1+\rho_{\mathrm{n}} e^{i \theta_{\mathrm{n}}} e^{i \gamma}-\left(1-\frac{2}{3} a_{\mathrm{C}} e^{i \Delta_{\mathrm{C}}}\right) q e^{i \phi} e^{i \omega} r_{\mathrm{c}} e^{i \delta_{\mathrm{c}}}\right]
\end{aligned}
$$

with

$$
\begin{aligned}
& \tilde{a}_{\mathrm{EW}}^{\mathrm{C}} e^{i \tilde{\psi}_{\mathrm{C}}} \equiv q e^{i \omega} r_{\mathrm{c}} e^{i \delta_{\mathrm{c}}} \tilde{a}_{\mathrm{C}} e^{i \tilde{\Delta}_{\mathrm{C}}} \\
& a_{\mathrm{EW}}^{\mathrm{C}} e^{i \psi_{\mathrm{C}}} \equiv q e^{i \omega} r_{\mathrm{c}} e^{i \delta_{\mathrm{c}}} a_{\mathrm{C}} e^{i \Delta_{\mathrm{C}}} .
\end{aligned}
$$

Because of the isospin relation in (D.15), the terms proportional to $\tilde{a}_{\mathrm{C}}$ and $a_{\mathrm{C}}$ have to cancel in the sums of the $A\left(B^{+} \rightarrow \pi^{+} K^{0}\right), \sqrt{2} A\left(B^{+} \rightarrow \pi^{0} K^{+}\right)$and $A\left(B_{d}^{0} \rightarrow \pi^{-} K^{+}\right)$, $\sqrt{2} A\left(B_{d}^{0} \rightarrow \pi^{0} K^{0}\right)$ amplitudes, respectively.

\section{D.3 The Case of $\phi=0^{\circ}$}

Let us first consider $\phi=0^{\circ}$, which applies also to the case of the SM. Although the analysis of the current $B$-factory data performed in Section 4 favours a large phase of $\phi \sim-90^{\circ}$, it is interesting and instructive to have a detailed look at the situation arising for $\phi=0^{\circ}$. In this case, we may straightforwardly absorb the terms in (D.18) and (D.19) proportional to $a_{\mathrm{C}} e^{i \Delta_{\mathrm{C}}}$ in the amplitude $P^{\prime}$, yielding

$$
\begin{aligned}
A\left(B_{d}^{0} \rightarrow \pi^{-} K^{+}\right) & =P^{\prime}\left[1-r e^{i \delta} e^{i \gamma}\right] \\
\sqrt{2} A\left(B_{d}^{0} \rightarrow \pi^{0} K^{0}\right) & =-P^{\prime}\left[1+\rho_{\mathrm{n}} e^{i \theta_{\mathrm{n}}} e^{i \gamma}-q e^{i \omega} r_{\mathrm{c}} e^{i \delta_{\mathrm{c}}}\right] .
\end{aligned}
$$


As far as (D.16) and (D.17) are concerned, we obtain amplitudes of the structure

$$
\begin{aligned}
A\left(B^{+} \rightarrow \pi^{+} K^{0}\right) & =-P^{\prime}\left[1+\rho_{\mathrm{c}} e^{i \theta_{\mathrm{c}}} e^{i \gamma}-a_{\mathrm{EW}}^{\mathrm{C}(1)} e^{i \psi_{\mathrm{C}}^{(1)}}\right] \\
\sqrt{2} A\left(B^{+} \rightarrow \pi^{0} K^{+}\right) & =P^{\prime}\left[1+\rho_{\mathrm{c}} e^{i \theta_{\mathrm{c}}} e^{i \gamma}-\left\{e^{i \gamma}-\left(1-a_{\mathrm{C}}^{(1)} e^{i \Delta_{\mathrm{C}}^{(1)}}\right) q e^{i \omega}\right\} r_{\mathrm{c}} e^{i \delta_{\mathrm{c}}}\right]
\end{aligned}
$$

with

$$
\begin{aligned}
a_{\mathrm{C}}^{(1)} e^{i \Delta_{\mathrm{C}}^{(1)}} & \equiv \frac{P_{\mathrm{EW}}^{\mathrm{C}(1)}}{P_{\mathrm{EW}}} \\
a_{\mathrm{EW}}^{\mathrm{C}(1)} e^{i \psi_{\mathrm{C}}^{(1)}} & \equiv q e^{i \omega} r_{\mathrm{c}} e^{i \delta_{\mathrm{c}}} a_{\mathrm{C}}^{(1)} e^{i \Delta_{\mathrm{C}}^{(1)}},
\end{aligned}
$$

where $P_{\mathrm{EW}}^{\mathrm{C}(1)}$ is given in (D.8). It should be noted that $P^{\prime}, r e^{i \delta}, \rho_{\mathrm{n}} e^{i \theta_{\mathrm{n}}}, r_{\mathrm{c}} e^{i \delta_{\mathrm{c}}}$ and $\rho_{\mathrm{c}} e^{i \theta_{\mathrm{c}}}$ now contain also contributions from colour-suppressed EW penguins. The expressions in (D.22) and (D.23) are the counterparts of (3.74) and (3.75), respectively.

We observe that the colour-suppressed EW penguins do not explicitly affect the analysis of the $B \rightarrow \pi \pi$ modes and the neutral $B \rightarrow \pi K$ decays. Because of this feature, $B_{d}^{0} \rightarrow \pi^{-} K^{+}$is actually the "natural" partner of $B_{d}^{0} \rightarrow \pi^{+} \pi^{-}$to deal with the famous penguin problem and not the $B^{+} \rightarrow \pi^{+} K^{0}$ channel, as is sometimes done in the literature. If we use the $B \rightarrow \pi \pi$ data as described above to fix the hadronic $B \rightarrow \pi K$ parameters, we may extract $q$ and $\omega$ from $R_{\mathrm{n}}$ and one of the CP-violating $B_{d} \rightarrow \pi^{0} K_{\mathrm{S}}$ observables; the remaining CP asymmetry provides an important consistency check of the $\phi=0^{\circ}$ scenario, in particular to see whether this weak phase actually vanishes. Interestingly, enhanced colour-suppressed EW penguins would yield a difference between the values of $r_{\mathrm{c}}$ following from (4.25) and (4.26), which is not indicated by the data.

If we fix the hadronic $B \rightarrow \pi K$ parameters through the $B \rightarrow \pi \pi$ system, the analysis of the charged $B \rightarrow \pi K$ modes may be affected both by the colour-suppressed EW penguins and by the $\rho_{\mathrm{c}}$ parameter. Following the avenue described above, we may predict the corresponding observables, and may check whether we obtain agreement with the experimental picture. Alternatively, we may use the value of $r_{\mathrm{c}}$ following from (4.26), and may shift the terms in (D.24) and (D.25) proportional to $a_{\mathrm{C}}^{(1)} e^{i \Delta_{\mathrm{C}}^{(1)}}$ into $P^{\prime}$. We may then also analyse the observables of the charged $B \rightarrow \pi K$ system in a manner that is not affected by the colour-suppressed EW penguins, whereas $\rho_{\mathrm{c}}$ may still enter.

\section{D.4 The Case of $\phi \neq 0^{\circ}$}

In the case of $\phi \neq 0^{\circ}$, we could of course still absorb colour-suppressed EW penguin contributions in the amplitude $P^{\prime}$. However, as these terms now contain a CP-violating weak phase, we have no longer the simple amplitude structure of $P^{\prime}=\left|P^{\prime}\right| e^{i \delta_{P^{\prime}}}$, where $\delta_{P^{\prime}}$ is a CP-conserving strong phase.

If we look at (D.16) and (D.18), we see that the observable $R$ and the direct CP asymmetries of the $B^{ \pm} \rightarrow \pi^{ \pm} K$ and $B_{d} \rightarrow \pi^{\mp} K^{ \pm}$modes would be significantly affected by the terms proportional to $\tilde{a}_{\mathrm{C}} e^{i \tilde{\Delta}_{\mathrm{C}}}$ and $a_{\mathrm{C}} e^{i \Delta_{\mathrm{C}}}$ should these coefficients not be small quantities, i.e. should the colour suppression of the EW penguins not be effective. However, as discussed in Subsection 4.4.1, these observables do not indicate any anomalous 
behaviour. Moreover, as we have already noted above, also the agreement between the values of $r_{\mathrm{c}}$ in (4.24) and (4.26) does not favour an enhancement of the colour-suppressed EW penguins. In this context, it is also important to note that the $B \rightarrow \pi K$ data do not require a dramatic enhancement of the parameter $q$. Moreover, the analysis of the rare decays in Section 5 favours $q$ to be even smaller than the central value given in (4.47).

In order to deal further with the colour-suppressed EW penguins in the $\phi \neq 0^{\circ}$ case, it is useful to introduce

$$
\langle q\rangle e^{i\langle\omega\rangle} \equiv\left[1-\frac{1}{2} a_{\mathrm{C}}^{(1)} e^{i \Delta_{\mathrm{C}}^{(1)}}\right] q e^{i \omega}
$$

so that

$$
\begin{aligned}
& {\left[1-\frac{1}{3} \tilde{a}_{\mathrm{C}} e^{i \tilde{\Delta}_{\mathrm{C}}}\right] q e^{i \omega}=\langle q\rangle e^{i\langle\omega\rangle}+\frac{1}{2} a_{\mathrm{C}}^{(0)} e^{i \Delta_{\mathrm{C}}^{(0)}} q e^{i \omega}} \\
& {\left[1-\frac{2}{3} a_{\mathrm{C}} e^{i \Delta_{\mathrm{C}}}\right] q e^{i \omega}=\langle q\rangle e^{i\langle\omega\rangle}-\frac{1}{2} a_{\mathrm{C}}^{(0)} e^{i \Delta_{\mathrm{C}}^{(0)}} q e^{i \omega},}
\end{aligned}
$$

with

$$
a_{\mathrm{C}}^{(0)} e^{i \Delta_{\mathrm{C}}^{(0)}} \equiv \frac{P_{\mathrm{EW}}^{\mathrm{C}(0)}}{P_{\mathrm{EW}}}
$$

in analogy to (D.26). Considering only tree-diagram-like matrix elements, as in (D.10) and (D.11), yields

$$
\left[\frac{a_{\mathrm{C}}^{(0)}}{a_{\mathrm{C}}^{(1)}}\right]_{\mathrm{T}}=\frac{1}{3}
$$

If we assume that also $a_{\mathrm{C}}^{(0)}$ is suppressed with respect to $a_{\mathrm{C}}^{(1)}$, we may identify the terms proportional to $q e^{i \omega}$ in (D.17) and (D.19) simply with the "effective" EW penguin parameter $\langle q\rangle e^{i\langle\omega\rangle}$. Interestingly, the strong phase $\Delta_{\mathrm{C}}^{(1)}$ may induce a sizeable value of $\langle\omega\rangle$, although $\omega$ could still be tiny. If we complement then

$$
\left[\frac{\mathrm{BR}\left(B^{+} \rightarrow \pi^{0} K^{+}\right)+\mathrm{BR}\left(\bar{B}^{-} \rightarrow \pi^{0} K^{-}\right)}{\operatorname{BR}\left(B_{d}^{0} \rightarrow \pi^{0} K^{0}\right)+\operatorname{BR}\left(\bar{B}_{d}^{0} \rightarrow \pi^{0} \bar{K}^{0}\right)}\right] \frac{\tau_{B_{d}^{0}}}{\tau_{B^{+}}} \equiv \frac{R_{\mathrm{c}}}{b}=0.99 \pm 0.15
$$

with two of the three CP-violating observables provided by the $B^{ \pm} \rightarrow \pi^{0} K^{ \pm}, B_{d} \rightarrow \pi^{0} K_{\mathrm{S}}$ modes, we may determine $\langle q\rangle,\langle\omega\rangle$ and $\phi$; the remaining third $\mathrm{CP}$ asymmetry can be predicted and allows a crucial consistency check.

To conclude, let us emphasize once more that our analysis of the current $B$-factory data for the $B \rightarrow \pi K$ modes performed in Section 4 points towards a consistent overall picture. In particular, $R$ and the direct CP asymmetries of the $B_{d} \rightarrow \pi^{\mp} K^{ \pm}, B^{ \pm} \rightarrow \pi^{ \pm} K$ modes do not show any anomalous behaviour. Consequently, we have no experimental indications for an enhancement of the colour-suppressed EW penguins, which have actually a very complicated internal structure. In the future, the strategies discussed in this appendix will allow us to explore these contributions in a more stringent manner with the help of improved experimental data. 


\section{References}

[1] M. Kobayashi and T. Maskawa, Prog. Theor. Phys. 49 (1973) 652.

[2] R. Fleischer, Phys. Rep. 370 (2002) 537.

[3] A.J. Buras, TUM-HEP-523-03 [hep-ph/0307203].

[4] M. Battaglia et al., hep-ph/0304132.

[5] Ya.I. Azimov, V.L. Rappoport and V.V. Sarantsev, Z. Phys. A356 (1997) 437;

Y. Grossman and H.R. Quinn, Phys. Rev. D56 (1997) 7259;

J. Charles et al., Phys. Lett. B425 (1998) 375;

B. Kayser and D. London, Phys. Rev. D61 (2000) 116012;

H.R. Quinn et al., Phys. Rev. Lett. 85 (2000) 5284;

A.S. Dighe, I. Dunietz and R. Fleischer, Phys. Lett. B433 (1998) 147;

I. Dunietz, R. Fleischer and U. Nierste, Phys. Rev. D63 (2001) 114015.

[6] R. Itoh, KEK-PREPRINT-2002-106 [hep-ex/0210025].

[7] B. Aubert et al. [BaBar Collaboration], BABAR-PUB-03-028 [hep-ex/0308012].

[8] K. Abe et al. [Belle Collaboration], BELLE-CONF-0354 [hep-ex/0308040].

[9] M. Beneke and M. Neubert, Nucl. Phys. B675 (2003) 333.

[10] M. Beneke, G. Buchalla, M. Neubert and C.T. Sachrajda, Phys. Rev. Lett. 83 (1999) 1914.

[11] Heavy Flavour Averaging Group, http://www.slac.stanford.edu/xorg/hfag/.

[12] A.J. Buras, R. Fleischer, S. Recksiegel and F. Schwab, CERN-TH/2003-314, to appear in Phys. Rev. Lett. [hep-ph/0312259].

[13] A.J. Buras and R. Fleischer, Eur. Phys. J. C11 (1999) 93.

[14] A.J. Buras and R. Fleischer, Eur. Phys. J. C16 (2000) 97.

[15] R. Fleischer and T. Mannel, Phys. Rev. D57 (1998) 2752.

[16] A.J. Buras, R. Fleischer, S. Recksiegel and F. Schwab, Eur. Phys. J. C32 (2003) 45.

[17] R. Fleischer and T. Mannel, TTP-97-22 [hep-ph/9706261].

[18] Y. Grossman, M. Neubert and A. Kagan, JHEP 9910 (1999) 029.

[19] T. Yoshikawa, Phys. Rev. D68 (2003) 054023.

[20] M. Gronau and J.L. Rosner, Phys. Lett. B572 (2003) 43. 
[21] D. London and R. Peccei, Phys. Lett. B223 (1989) 257;

N.G. Deshpande and J. Trampetic, Phys. Rev. D41 (1990) 895 and 2926;

J.-M. Gérard and W.-S. Hou, Phys. Rev. D43 (1991) 2909.

[22] R. Fleischer, Z. Phys. C62 (1994) 81.

[23] N.G. Deshpande and X.-G. He, Phys. Lett. B336 (1994) 471.

[24] T. Browder, hep-ex/0312024, talk at Lepton-Photon 2003, Fermilab, Batavia, Illinois, 11-16 August 2003, http://conferences.fnal.gov/lp2003/.

[25] K. Abe et al. [Belle Collaboration], hep-ex/0308035.

[26] Y. Grossman and M. Worah, Phys. Lett. B395 (1997) 241.

[27] R. Fleischer, Int. J. Mod. Phys. A12 (1997) 2459.

[28] D. London and A. Soni, Phys. Lett. B407 (1997) 61.

[29] R. Fleischer and T. Mannel, Phys. Lett. B511 (2001) 240.

[30] Y. Grossman, Z. Ligeti, Y. Nir and H.R. Quinn, Phys. Rev. D68 (2003) 015004.

[31] A.J. Buras and L. Silvestrini, Nucl. Phys. B546 (1999) 299.

[32] A.J. Buras, A. Romanino and L. Silvestrini, Nucl. Phys. B520 (1998) 3.

[33] A.J. Buras, G. Colangelo, G. Isidori, A. Romanino and L. Silvestrini, Nucl. Phys. B566 (2000) 3.

[34] G. Buchalla, G. Hiller and G. Isidori, Phys. Rev. D63 (2001) 014015;

D. Atwood and G. Hiller, LMU-09-03 [hep-ph/0307251].

[35] G. Buchalla, A.J. Buras and M.E. Lautenbacher, Rev. Mod. Phys. 68 (1996) 1125.

[36] G. Buchalla, A.J. Buras and M.K. Harlander, Nucl. Phys. B349 (1991) 1.

[37] A.J. Buras and M.K. Harlander, Adv. Ser. Direct. High Energy Phys. 10 (1992) 58.

[38] T. Inami and C.S. Lim, Progr. Theor. Phys. 65 (1981) 297.

[39] A.J. Buras, TUM-HEP-530-03 [hep-ph/0310208].

[40] A.J. Buras, P. Gambino, M. Gorbahn, S. Jäger and L. Silvestrini, Phys. Lett. B500 (2001) 161.

[41] G. D'Ambrosio, G.F. Giudice, G. Isidori and A. Strumia, Nucl. Phys. B645 (2002) 155.

[42] C. Bobeth, T. Ewerth, F. Krüger and J. Urban, Phys. Rev. D66 (2002) 074021. 
[43] Y. Grossman, Y. Nir and R. Rattazzi, Adv. Ser. Direct. High Energy Phys. 15 (1998) 755.

[44] V. Barger, C.W. Chiang, P. Langacker and H.S. Lee, Phys. Lett. B580 (2004) 186.

[45] M. Neubert and J.L. Rosner, Phys. Lett. B441 (1998) 403; Phys. Rev. Lett. 81 (1998) 5076.

[46] R. Fleischer, Eur. Phys. J. C6 (1999) 451.

[47] A.J. Buras, M. Jamin and M.E. Lautenbacher, Nucl. Phys. B408 (1993) 209.

[48] M. Neubert, JHEP 9902 (1999) 014.

[49] L. Wolfenstein, Phys. Rev. Lett. 51 (1983) 1945.

[50] A.J. Buras, M.E. Lautenbacher and G. Ostermaier, Phys. Rev. D50 (1994) 3433.

[51] M. Gronau and D. London, Phys. Rev. Lett. 65 (1990) 3381.

[52] M. Gronau, Phys. Lett. B300 (1993) 163;

R. Fleischer and T. Mannel, Phys. Lett. B397 (1997) 269;

Y. Grossman and H.R. Quinn, Phys. Rev. D58 (1998) 017504;

J. Charles, Phys. Rev. D59 (1999) 054007;

M. Gronau, D. London, N. Sinha and R. Sinha, Phys. Lett. B514 (2001) 315.

[53] R. Fleischer, Phys. Lett. B459 (1999) 306.

[54] R. Fleischer, Eur. Phys. J. C16 (2000) 87.

[55] R. Fleischer and J. Matias, Phys. Rev. D66 (2002) 054009.

[56] R. Fleischer, G. Isidori and J. Matias, JHEP 0305 (2003) 053.

[57] G. Buchalla and A.S. Safir, LMU-25-03 [hep-ph/0310218];

F.J. Botella and J.P. Silva, hep-ph/0312337.

[58] M. Gronau, O.F. Hernandez, D. London and J.L. Rosner, Phys. Rev. D52 (1995) 6374 .

[59] R. Fleischer, Phys. Lett. B365 (1996) 399.

[60] M. Gronau, D. Pirjol and T.M. Yan, Phys. Rev. D60 (1999) 034021.

[61] R. Fleischer, Z. Phys. C58 (1993) 483.

[62] A.J. Buras, R. Fleischer and T. Mannel, Nucl. Phys. B533 (1998) 3.

[63] A.J. Buras and L. Silvestrini, Nucl. Phys. B569 (2000) 3.

[64] A.J. Buras and R. Fleischer, Phys. Lett. B341 (1995) 379. 
[65] H. Jawahery, talk at Lepton-Photon 2003, Fermilab, Batavia, Illinois, 11-16 August 2003, http://conferences.fnal.gov/lp2003/.

[66] K. Abe et al. [Belle Collaboration], Phys. Rev. D68 (2003) 012001.

[67] K. Abe et al. [Belle Collaboration], Belle preprint 2004-1 [hep-ex/0401029].

[68] M. Ciuchini, E. Franco, G. Martinelli, L. Silvestrini, Nucl. Phys. B501 (1997) 271; C. Isola, M. Ladisa, G. Nardulli, T.N. Pham and P. Santorelli, Phys. Rev. D64 (2001) 014029 and D65 (2002) 094005;

M. Ciuchini, E. Franco, G. Martinelli, M. Pierini and L. Silvestrini, Phys. Lett. B515 (2001) 33.

[69] R. Fleischer, Phys. Lett. B341 (1994) 205.

[70] G. Wilkinson, private communication.

[71] K. Anikeev et al., FERMILAB-Pub-01/197 [hep-ph/0201071].

[72] P. Ball et al., CERN-TH/2000-101 [hep-ph/0003238], in CERN Report on Standard Model physics (and more) at the LHC (CERN, Geneva, 2000) p. 305.

[73] G. Balbi et al., CERN-LHCb/2003-123 and 124.

[74] J.P. Silva and L. Wolfenstein, Phys. Rev. D49 (1994) 1151.

[75] R. Fleischer, Nucl. Phys. B671 (2003) 459.

[76] N.G. Deshpande and X.-G. He, Phys. Rev. Lett. 74 (1995) 26 [E: ibid. 74 (1995) 4099].

[77] M. Gronau and J.L. Rosner, CLNS-03-1852 [hep-ph/0311280].

[78] A.J. Buras and R. Fleischer, Phys. Lett. B365 (1996) 390.

[79] H.J. Lipkin, Y. Nir, H.R. Quinn and A. Snyder, Phys. Rev. D44 (1991) 1454.

[80] A.F. Falk, A.L. Kagan, Y. Nir and A.A. Petrov, Phys. Rev. D57 (1998) 4290.

[81] M. Beneke, G. Buchalla, M. Neubert and C.T. Sachrajda, Nucl. Phys. B606 (2001) 245.

[82] M. Gronau, J.L. Rosner and D. London, Phys. Rev. Lett. 73 (1994) 21.

[83] A. Khodjamirian, T. Mannel and M. Melcher, Phys. Rev. D68 (2003) 114007.

[84] M. Bauer, B. Stech and M. Wirbel, Z. Phys. C34 (1987) 103.

[85] A.J. Buras, F. Parodi and A. Stocchi, JHEP 0301 (2003) 029.

[86] H.J. Lipkin, Phys. Lett. B445 (1999) 403. 
[87] M. Gronau, Y. Grossman and J.L. Rosner, Phys. Lett. B579 (2004) 331.

[88] M. Gronau and J.L. Rosner, Phys. Lett. B482 (2000) 71.

[89] K. Hagiwara et al. [Particle Data Group], Phys. Rev. D66 (2002) 010001.

[90] J. Kaneko et al. [Belle Collaboration], Phys. Rev. Lett. 90 (2003) 021801;

B. Aubert et al. [BaBar Collaboration], hep-ex/0308016.

[91] A. Alavi-Harati et al. [KTeV Collaboration], hep-ex/0309072.

[92] G. Isidori, hep-ph/0307014 and references therein.

[93] G. Buchalla and A.J. Buras, Nucl. Phys. B400 (1993) 225, Nucl. Phys. B412 (1994) 106.

[94] G. Buchalla and A.J. Buras, Nucl. Phys. B548 (1999) 309.

[95] M. Misiak and J. Urban, Phys. Lett. B541 (1999) 161.

[96] G. Buchalla and A.J. Buras, Phys. Lett. B333 (1994) 221, Phys. Rev. D54 (1996) 6782.

[97] A.J. Buras and R. Fleischer, Phys. Rev. D64 (2001) 115010.

[98] S. Kettell, L. Landsberg and H. Nguyen, hep-ph/0212321.

[99] S. Adler et al., Phys. Rev. Lett. 79 (1997) 2204, Phys. Rev. Lett. 84 (2000) 3768, Phys. Rev. Lett. 88 (2002) 041803.

[100] A. Alavi-Harati et al., Phys. Rev. D61 (2000) 072006.

[101] Y. Grossman and Y. Nir, Phys. Lett. B398, (1997) 163.

[102] G. Buchalla and G. Isidori, Phys. Lett. B440 (1998) 170.

[103] A.J. Buras, Phys. Lett. B566 (2003) 115.

[104] M. Nakao, talk at Lepton-Photon 2003, Fermilab, Batavia, Illinois, 11-16 August 2003, http://conferences.fnal.gov/lp2003/.

[105] M.-C. Chang et al. [Belle Collaboration], hep-ex/0309069.

[106] G. D’Ambrosio, G. Isidori and J. Portolés, Phys. Lett. B423 (1998) 385;

G. Isidori and A. Retico, JHEP 0209 (2002) 063.

[107] D. Gomez Dumm and A. Pich, Nucl. Phys. Proc. Suppl. 74 (1999) 186;

G. Valencia, hep-ph/9711377;

M. Knecht, S. Peris, M. Perrottet and E. de Rafael, Phys. Rev. Lett. 83 (1999) 5230 ;

D. Greynat and E. de Rafael, hep-ph/0303096 and references therein. 
[108] G. Hiller and F. Krüger, TUM-HEP-519-03 [hep-ph/0310219].

[109] H.H. Asatrian, H.M. Asatrian, C. Greub and M. Walker, Phys. Lett. B507 (2001) 162, Phys. Rev. D65 (2002) 074004, Phys. Rev. D66 (2002) 034009;

H.M. Asatrian, K. Bieri, C. Greub and A. Hovhannisyan, Phys. Rev. D66 (2002) 094013.

[110] A. Ghinculov, T. Hurth, G. Isidori and Y.P. Yao, Nucl. Phys. B648 (2003) 254, hep-ph/0211197, hep-ph/0310187.

[111] P. Gambino, M. Gorbahn and U. Haisch, Nucl. Phys. B673 (2003) 238;

C. Bobeth, P. Gambino, M. Gorbahn and U. Haisch, hep-ph/0312090.

[112] T. Hurth, Rev. Mod. Phys. 75 (2003) 1159;

G. Isidori, hep-ph/0401079.

[113] A. Ali, E. Lunghi, C. Greub and G. Hiller, Phys. Rev. D66 (2002) 034002.

[114] M. Misiak, Nucl. Phys. B393 (1993) 23 [E: Nucl. Phys. B439 (1995) 461].

[115] A.J. Buras and M. Münz, Phys. Rev. D 52 (1995) 186.

[116] A. Ali, T. Mannel and T. Morozumi, Phys. Lett. B273 (1991) 505.

[117] G. Burdman, Phys. Rev. D57 (1998) 4254.

[118] G. Buchalla, G. D’Ambrosio and G. Isidori, Nucl. Phys. B672 (2003) 387.

[119] A. Lai et al., Phys. Lett. B556 (2003) 105;

C. Lazzeroni, talk at the HEP 2003 Europhysics Conference, Aachen, Germany, 17- 23 July 2003, http://eps2003.physik.rwth-aachen.de/.

[120] A.J. Buras, M.E. Lautenbacher, M. Misiak and M. Münz, Nucl. Phys. B423 (1994) 349.

[121] A.J. Buras and M. Jamin, hep-ph/0306217.

[122] A. Lai et al., Eur. Phys. J. C22 (2001) 231;

J.R. Batley et al., Phys. Lett. B544 (2002) 97.

[123] A. Alavi-Harati et al., Phys. Rev. Lett. 83 (1999) 22, Phys. Rev. D67 (2003) 012005.

[124] J. Bijnens and J. Prades, JHEP 06 (2000) 035, Nucl. Phys. Proc. Suppl. 96 (2001) 354 ;

J. Bijnens, E. Gamiz and J. Prades, JHEP 10 (2001) 009.

[125] M. Knecht, S. Peris and E. de Rafael, Phys. Lett. B508 (2001) 117;

T. Hambye, S. Peris and E. de Rafael, JHEP 0305 (2003) 027. 
[126] A.J. Buras, hep-ph/9806471, in the proceedings of the Les Houches 1997 Summer School on Theoretical Physics: Probing the Standard Model of Particle Interactions, July 28 - September 5, 1997, Les Houches, France, eds. R. Gupta, A. Morel, E. de Rafael and F. David, North Holland, Amsterdam (1998).

[127] R. Fleischer and T. Mannel, Phys. Lett. B506 (2001) 311.

[128] R. Fleischer, Phys. Lett. B562 (2003) 234; Nucl. Phys. B659 (2003) 321.

[129] Y.-Y. Keum, H.-N. Li and A.I. Sanda, Phys. Lett. B504 (2001) 6; Phys. Rev. D63 (2001) 054008 and references therein.

[130] C.W. Bauer, D. Pirjol and I.W. Stewart, Phys. Rev. D65 (2002) 054022;

C.W. Bauer, S. Fleming, D. Pirjol, I.Z. Rothstein and I.W. Stewart, Phys. Rev. D66 (2002) 014017;

C.W. Bauer, D. Pirjol and I.W. Stewart, Phys. Rev. D66 (2002) 054005;

I.W. Stewart, hep-ph/0208034 and references therein. 